\title{
Decomposition theorem and Riesz basis for axisymmetric potentials in the right half-plane
}

\author{
Slah Chaabi ${ }^{1}$ - Stéphane Rigat ${ }^{1}$
}

Received: 9 May 2014 / Revised: 27 February 2015/ Accepted: 17 May 2015 /

Published online: 14 July 2015

(C) Springer International Publishing AG 2015

\begin{abstract}
We consider the Weinstein equation, also known as the equation governing generalized axisymmetric potentials (GASP), with complex coefficients $L_{m} u=\Delta u+$ $(m / x) \partial_{x} u=0, m \in \mathbb{C}$. We generalize results known for $m \in \mathbb{R}$ to the case $m \in \mathbb{C}$. In particular, explicit expressions of fundamental solutions for Weinstein operators and their estimates near singularities are presented, a Green's formula for GASP in the right half-plane $\mathbb{H}^{+}$for $\operatorname{Re} m<1$ is established. We prove a new decomposition theorem for the GASP in annular domains for $m \in \mathbb{C}$, which is in fact a generalization of the Bôcher's decomposition theorem. In particular, using bipolar coordinates, it is proved for annuli that a family of solutions for the GASP equation in terms of associated Legendre functions of first and second kind is complete. This family is shown to be a Riesz basis in some non-concentric circular annuli.
\end{abstract}

Keywords Axially symmetric solutions · Fundamental solutions · Riesz basis · Elliptic functions and integrals

Mathematics Subject Classification $35 \mathrm{~B} 07 \cdot 33 \mathrm{E} 05 \cdot 35 \mathrm{~J} 15 \cdot 35 \mathrm{E} 05 \cdot 42 \mathrm{C} 15$

Originally considered by the Central European Journal of Mathematics but withdrawn due to imposition of publishing fees and resubmitted to the European Journal of Mathematics.

Stéphane Rigat

stephane.rigat@univ-amu.fr

Slah Chaabi

slah.chaabi@univ-amu.fr

1 I2M, UMR 7373, Aix-Marseille Université, CMI, rue Frédéric Joliot-Curie no 39, 13453 Marseille, France 


\section{Introduction}

In this article, we study the Weinstein differential operator

$$
L_{m}=x^{-m} \operatorname{div}\left(x^{m} \nabla \cdot\right)=\Delta+\frac{m}{x} \frac{\partial}{\partial x}, \quad m \in \mathbb{C},
$$

well-defined on the right half-plane $\mathbb{H}^{+}=\left\{(x, y) \in \mathbb{R}^{2}: x>0\right\}=\{z \in \mathbb{C}:$ $\operatorname{Re} z>0\}$ with the convention $x^{m}=\exp (m \ln x)$. This class of operators is also called operators governing axisymmetric potentials. They have been studied quite extensively in cases $m \in \mathbb{N}$ and $m \in \mathbb{R}$ in [10-13,16-18,22-24,29-33,36-38,41,5168]. We will focus exclusively on the case $m \in \mathbb{C}$, recalling in the course of the paper some results for integer values of $m$. The Weinstein equation reads

$$
L_{m} u=0 .
$$

The main motivation for which we consider the case $m \in \mathbb{C}$ is that if we complexify the coordinates by writing $z=x+i y,(1)$ takes the form

$$
\frac{\partial^{2} u}{\partial z \partial \bar{z}}+\frac{m / 2}{z+\bar{z}}\left(\frac{\partial u}{\partial z}+\frac{\partial u}{\partial \bar{z}}\right)=0,
$$

which is a particular case of the equation

$$
\frac{\partial^{2} u}{\partial z \partial \bar{z}}+\frac{\alpha}{z+\bar{z}} \frac{\partial u}{\partial z}+\frac{\beta}{z+\bar{z}} \frac{\partial u}{\partial \bar{z}}=0
$$

considered with $\alpha, \beta \in \mathbb{C}$ in [49, Equation (5.7), p. 20].

Equation (1) also appears in physics in the study of the behavior of plasma in a tokamak. The role of tokamak, which has a toroidal geometry, is to control location of the plasma in its chamber by applying magnetic fields on its boundary. It is possible to assume that plasma is axially symmetric what reduces this problem to a plane section in $\mathbb{H}^{+}$, where the magnetic flux in the vacuum between the plasma and the circular boundary of the chamber satisfies a second-order elliptic nonlinear partial differential equation, the so-called Grad-Shafranov equation, which reduces to the homogeneous equation (1) with $m=-1$ (Fig. 1).

Note that in this instance, (1) takes place in an annular domain rather than in a simply connected domain, see $[8,9,46]$. This fact motivates our decomposition theorem, Theorem 5.9.

In the sequel, the sense in which the solutions are studied will be specified. We will also look at solutions to the equation in the sense of distributions

$$
L_{m} u=\delta_{(x, y)},
$$

where $\delta_{(x, y)}$ denotes the Dirac mass at $(x, y) \in \mathbb{H}^{+}$. 


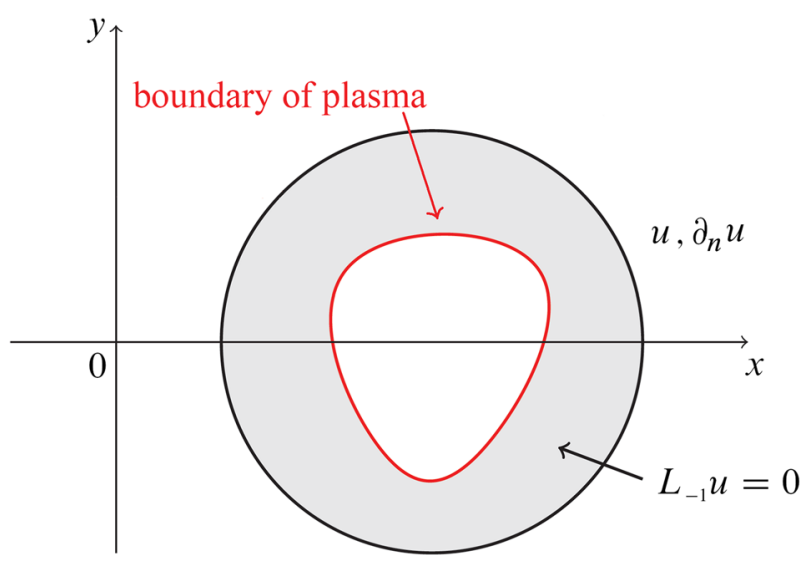

Fig. 1 Section of a Tokamak

The mentioned above class of operators was first considered by Weinstein in [54], where he studied the case $m \in \mathbb{N}^{*}$. He also established a relation between the axisymmetric potentials for $m \in \mathbb{N}^{*}$ and harmonic functions on $\mathbb{R}^{m+2}$, see Proposition 2.4.

In [20,58,59], Weinstein and Diaz-Weinstein established the correspondence principle between axisymmetric potentials $L_{m}$ and $L_{2-m}$, see Proposition 2.3. They deduced an expression of a fundamental solution (where the singular point is taken on the $y$-axis) for $m \in \mathbb{R}$ and established a link between the Weinstein equation and Tricomi equations and their fundamental solutions.

Let us return to the book [49]. Studying elliptic equations with analytic coefficients, Vekua provided means to express their fundamental solutions by using the Riemann functions, introduced earlier (see e.g. [28]) in the real hyperbolic context, he also investigated generalized elliptic equations with complex operators $\partial_{z}$ and $\partial_{\bar{z}}$. In heuristic words, in the same way as a harmonic function is the real part of a holomorphic function, or the sum of a holomorphic and an anti-holomorphic function, Vekua established the fact that solutions to elliptic equations, and therefore GASP, can be written as a sum of two functionals, one applied to an arbitrary holomorphic function and the other applied to an arbitrary anti-holomorphic function. These functionals can be written explicitly in terms of the Riemann function, by using the hypergeometric functions [49] or fractional derivations [16]. In [34], Henrici gave a very interesting introduction to the work of Vekua.

More recently, basing on the work of Vekua, Savina [44] gave a series representation of fundamental solutions for the operator $\widehat{L} u=\Delta u+a \partial_{x} u+b \partial_{y} u+c u$ and studied the convergence of this series. She also provided an application of her results to the Helmholtz equation.

In [31], Gilbert studied the non-homogeneous Weinstein equation, i.e. the case $m \geq 0$, and gave an integral formula for this class of equations. In particular, an explicit solution was given when the second member depends only on one variable.

Some Dirichlet problems are considered in $[40,41]$ in a special geometry, the socalled "geometry with separable variable". 
Even if some results presented in this paper are known for real values of $m$, we make a totally self-contained presentation involving elementary technics not necesseraly used in the papers mentioned above. For instance, usual arguments involving estimates of hypergeometric integrals are replaced by arguments using the Lebesgue dominated convergence theorem. Our main result is a decomposition theorem for axisymmetric potentials which is new also for real values of $m$. We obtain a Liouville-type result for the solutions of Weinstein equation on $\mathbb{H}^{+}$, with an interesting observation that there is a loss of strict ellipticity of the Weinstein operator on the boundary of $\mathbb{H}^{+}$. An application of the decomposition theorem is given by showing that an explicit family of axisymmetric potentials constructed by introduction of bipolar coordinates is a Riesz basis in some annuli.

The plan of the paper is the following. In Sect. 2, we recall preliminary information about fundamental solutions for linear partial differential operators with non-constant coefficients. Proposition 2.1 provides with a connection between fundamental solutions for $L_{m}$ and fundamental solutions for $L_{m}^{\star}$, where $L_{m}^{\star}$ denotes the formal adjoint of $L_{m}$. The Weinstein principle [59], valid for $m$ real and complex, establishes a connection between $L_{m}$ and $L_{2-m}$. We state it without proof as Proposition 2.3. Proposition 2.4, valid only for $m \in \mathbb{N}$, is fundamental in the sense that one can compute a fundamental solution for $L_{m}$ just knowing the usual fundamental solution for the Laplacian in $\mathbb{R}^{m+2}$. The corresponding computations are done for $m \in \mathbb{N}$ and for $m \in \mathbb{Z}$ in Sect. 3 .

The extension of formulas for fundamental solutions to the case $m \in \mathbb{C}$ is the core of Sect. 4. First we describe in Proposition 4.2 in an elementary way the behavior of fundamental solutions near their singularities. Next we use the corresponding estimates to establish the main result of the section, Theorem 4.4.

Section 5 is dedicated to the decomposition theorem. First we modify the fundamental solutions built earlier in order to get fundamental solutions which vanish on the boundary of $\mathbb{H}^{+}$. Next, in Proposition 5.3, we show that if $u$ is a solution for $L_{m} u=0$ which vanishes on the boundary of $\mathbb{H}^{+}$, then $u \equiv 0$ on $\mathbb{H}^{+}$. Let us emphasize here that, although this statement looks obvious, this is not the case due to the loss of ellipticity of $L_{m}$ on the boundary of $\mathbb{H}^{+}$. Let us mention that Proposition 5.3 is a consequence of the maximum principle for pseudo-analytic functions given in a recent paper by Chalendar-Partington [14] for more general function $\sigma$ than $x^{m}$, but in [14] there is an additional assumption on $\sigma$, which in our case corresponds to the assumption $|m| \geq 1$. The proof of Proposition 5.3 is quite long, but not difficult, it follows careful estimates of fundamental solutions in some parts of $\mathbb{H}^{+}$. Finally the decomposition theorem, Theorem 5.9, is proved. Its proof is similar to the proof of the Bôcher's decomposition theorem presented in [4]. We end Sect. 5 with a Poisson formula for axisymmetric potentials in $\mathbb{H}^{+}$, Proposition 5.10.

In Sect. 6, we consider the case where the annular domain is a kind of annulus. We introduce very classical (in physics) bipolar coordinates, cf. [39], in which the GASP equation has a form presented in Theorem 6.2. Next, applying the method of separation of variables we obtain a basis of solutions in disks and complements of disks in $\mathbb{H}^{+}$, see Theorem 6.3. In Sect. 7 it is shown that this basis forms a Riesz basis. 


\section{Notations and preliminaries}

Throughout $\mathbb{H}^{+}=\left\{(x, y) \in \mathbb{R}^{2}: x>0\right\}$ stands for the right half-plane, all scalar functions are assumed to be complex valued. If $\Omega$ is an open set in $\mathbb{R}^{n}, n \in \mathbb{N}^{*}$, let $\mathcal{D}(\Omega)$ designate the space of $C^{\infty}$-functions compactly supported on $\Omega$, where supp $f=\overline{\{x \in \Omega: f(x) \neq 0\}}$. If $K$ is a compact set in $\Omega$, let $\mathcal{D}_{K}(\Omega)$ be the set of functions $\varphi \in \mathcal{D}(\Omega)$ such that $\operatorname{supp} \varphi \subset K$.

The partial derivatives of a differentiable function $u$ on an open set $\Omega \subset \mathbb{R}^{n}$ will be denoted by $\partial u / \partial x_{i}, \partial_{x_{i}} u$ or $u_{x_{i}}, i \in\{1, \ldots, n\}$. If $\alpha=\left(\alpha_{1}, \ldots, \alpha_{n}\right) \in \mathbb{N}^{n}$ is a multi-index, we denote

$$
\partial^{\alpha}=\partial_{x_{1}}^{\alpha_{1}} \ldots \partial_{x_{n}}^{\alpha_{n}}=\frac{\partial^{|\alpha|}}{\partial x_{1}^{\alpha_{1}} \ldots \partial x_{n}^{\alpha_{n}}}
$$

with $|\alpha|=\alpha_{1}+\cdots+\alpha_{n}$.

It is assumed that the reader is familiar with the terminology of distributions and we refer to [35].

Let $L$ be a linear differential operator on $\Omega$,

$$
L=\sum_{|\alpha| \leq N} a_{\alpha} \partial^{\alpha}, \quad N \in \mathbb{N}
$$

where the summation runs over the multi-indices $\alpha$ of length $|\alpha| \leq N, a_{\alpha}$ are $C^{\infty}(\Omega)$ functions. If $T$ is a distribution, then $L T=\sum_{|\alpha| \leq N} a_{\alpha} \partial^{\alpha} T$. Denote by $L^{\star}$ the adjoint operator of $L$ in the sense of distributions, namely,

$$
L^{\star} T=\sum_{|\alpha| \leq N}(-1)^{|\alpha|} \partial^{\alpha}\left(a_{\alpha} T\right) .
$$

One can easily check, if $f, g \in \mathcal{D}(\Omega)$, we have

$$
\langle L f, g\rangle=\left\langle f, L^{\star} g\right\rangle .
$$

Let $a \in \Omega$ and $L$ be a differential operator on $\Omega$. A fundamental solution for $L$ on $\Omega$ at $a \in \Omega$ is a distribution $T_{a}$ such that

$$
L T_{a}=\delta_{a}
$$

where the equality is understood in the sense of distributions on $\Omega$. This equality can be rewritten as

$$
\varphi(a)=\left\langle L T_{a}, \varphi\right\rangle=\left\langle T_{a}, L^{\star} \varphi\right\rangle, \quad \varphi \in \mathcal{D}(\Omega)
$$

In particular, if $a \in \Omega$ and $T_{a}$ is a fundamental solution to $L^{\star}$ at $a$ on $\Omega$ and if $g \in \mathcal{D}(\Omega)$ is such that $g=L(\varphi)$ with $\varphi \in \mathcal{D}(\Omega)$, then 


$$
\varphi(a)=\left\langle T_{a}, g\right\rangle, \quad a \in \Omega .
$$

Indeed, we have

$$
\varphi(a)=\left\langle\delta_{a}, \varphi\right\rangle=\left\langle L^{\star} T_{a}, \varphi\right\rangle=\left\langle T_{a}, L \varphi\right\rangle=\left\langle T_{a}, g\right\rangle, \quad a \in \Omega .
$$

These fundamental solutions are therefore a good tool for solving equations $L \varphi=g$ on $\mathcal{D}(\Omega)$ if $g \in \mathcal{D}(\Omega)$.

If $m \in \mathbb{N}^{*}$, the Laplacian in $\mathbb{R}^{m}$ will be denoted by $\Delta_{m}$, or $\Delta$ when $m=2$. For $m \in \mathbb{C}, L_{m}$ denotes the Weinstein operator:

$$
L_{m} u(x, y)=\Delta u(x, y)+\frac{m}{x} \frac{\partial u}{\partial x}(x, y), \quad u \in C^{2}\left(\mathbb{H}^{+}\right), \quad(x, y) \in \mathbb{H}^{+}
$$

If $f(x, y)=\left(f_{1}(x, y), f_{2}(x, y)\right)$ is a $C^{1}$-vector $\mathbb{C}^{2}$-valued function on an open set in $\mathbb{R}^{2}$

$$
\operatorname{div} f=\frac{\partial f_{1}}{\partial x}+\frac{\partial f_{2}}{\partial y}
$$

Similarly, if $f: \mathbb{R}^{2} \rightarrow \mathbb{C}$ is a $C^{1}$-scalar $\mathbb{C}^{2}$-valued function on an open set in $\mathbb{R}^{2}$,

$$
\nabla f=\left(\frac{\partial f}{\partial x}, \frac{\partial f}{\partial y}\right)
$$

With these notations, the operator $L_{m}$ can be written as follows:

$$
L_{m} u(x, y)=x^{-m} \operatorname{div}\left(x^{m} \nabla u\right)(x, y), \quad u \in C^{2}\left(\mathbb{H}^{+}\right) .
$$

By the Schwarz rule, if $u$ is a function defined on a connected open set in $\mathbb{H}^{+}$such that $\operatorname{div}(\sigma \nabla u)=0$, where $\sigma: \mathbb{H}^{+} \rightarrow \mathbb{C}^{*}$ is a $C^{1}$-function, then there is a function $v$ which satisfies the well-known generalized Cauchy-Riemann system of equations

$$
\left\{\begin{array}{l}
\frac{\partial v}{\partial x}=-\sigma \frac{\partial u}{\partial y} \\
\frac{\partial v}{\partial y}=\sigma \frac{\partial u}{\partial x}
\end{array}\right.
$$

and $v$ satisfies the conjugate equation $\operatorname{div}(1 / \sigma \cdot \nabla v)=0$, see for example [7]. This observation justifies the fact that we call $L_{-m}, m \in \mathbb{C}$, the conjugate operator of $L_{m}$.

The adjoint operator of $L_{m}$ is

$L_{m}^{\star} u(x, y)=\Delta u(x, y)-\frac{\partial}{\partial x} \frac{m u(x, y)}{x}=\Delta u(x, y)-\frac{m}{x} \frac{\partial u}{\partial x}(x, y)+\frac{m}{x^{2}} u(x, y)$,

where $u \in C^{2}\left(\mathbb{H}^{+}\right),(x, y) \in \mathbb{H}^{+}$. This definition is given on $\mathbb{H}^{+}$but it is easily transposed to the case of an open set $\Omega$ of $\mathbb{H}^{+}$. 
In the case where the functions involved do not depend only on $x$ and $y$, we will write $L_{m, x, y}$ instead of $L_{m}$, which means that the partial derivatives are related to the variables $x$ and $y$, and all other variables are considered to be fixed.

If $u \in \mathcal{D}\left(\mathbb{H}^{+}\right)$, we define $S_{m} u, D u \in \mathcal{D}\left(\mathbb{H}^{+}\right)$as

$$
\left(S_{m} u\right)(x, y)=x^{-m} u(x, y), \quad(D u)(x, y)=\frac{\partial u}{\partial x}(x, y) .
$$

These operators satisfy the following property.

Proposition 2.1 The operator $S_{m}$ conjugates $L_{m}^{\star}$ and $L_{m}$, D conjugates $L_{-m}^{\star}$ and $L_{m}$, which means that

$$
S_{m} L_{m}^{\star}=L_{m} S_{m}, \quad L_{-m}^{\star} D=D L_{m}
$$

Remark 2.2 1. Let $m \in \mathbb{C}, S_{m}$ and $L_{m} S_{m}$ are self-adjoint operators, i.e. $S_{m}=S_{m}^{\star}$ and $L_{m} S_{m}=\left(L_{m} S_{m}\right)^{\star}$.

2. Let $\sigma: \Omega \rightarrow \mathbb{C}$ be a $C^{1}$-function which does not vanish, consider the operator defined on $C^{2}(\Omega)$ as follows:

$$
P_{\sigma} u(x, y)=\frac{1}{\sigma(x, y)} \operatorname{div}(\sigma(x, y) \nabla u(x, y)), \quad u \in C^{2}(\Omega)
$$

where $\Omega$ is an open set in $\mathbb{R}^{2}$. Then

$$
P_{\sigma}^{\star}=\operatorname{div}\left(\sigma \nabla\left(\frac{\dot{\sigma}}{\sigma}\right)\right)
$$

Indeed, if $u, v \in \mathcal{D}(\Omega)$, then, by using the derivation in the sense of distributions, we have

$$
\begin{aligned}
\left\langle P_{\sigma} u, v\right\rangle & =\int_{\Omega} \frac{1}{\sigma(x, y)} \operatorname{div}(\sigma(x, y) \nabla u(x, y)) v(x, y) d x d y \\
& =-\int_{\Omega} \sigma \nabla u \cdot \nabla\left(\frac{v}{\sigma}\right) d x d y=\int_{\Omega} u \operatorname{div}\left(\sigma \nabla\left(\frac{v}{\sigma}\right)\right) d x d y \\
& =\left\langle u, P_{\sigma}^{\star} v\right\rangle .
\end{aligned}
$$

Define $S_{\sigma}$ as

$$
\left(S_{\sigma} u\right)(x, y)=\frac{1}{\sigma(x, y)} u(x, y), \quad u \in C^{2}(\Omega)
$$

Then, one can easily check that $S_{\sigma} P_{\sigma}^{\star}=P_{\sigma} S_{\sigma}$, hence $S_{\sigma}$ conjugates $P_{\sigma}$ and $P_{\sigma}^{\star}$. The operators $P_{\sigma}$ and $S_{\sigma}$ are a generalization of $L_{m}$ and $S_{m}$ with the conjugation relation preserved.

If $m$ is a positive integer, introduce an operator $T_{m}: u \mapsto v$ defined as follows: $T_{m}$ maps a function $u$ defined on an open set $\Omega$ of $\mathbb{H}^{+}$to the function 


$$
v\left(x_{1}, \ldots, x_{m+2}\right)=u\left(\sqrt{x_{1}^{2}+\cdots+x_{m+1}^{2}}, x_{m+2}\right) .
$$

The following two propositions can be found in the Weinstein paper [59] in the case $m \in \mathbb{R}$. They can be checked by a direct computation for $m$ real and complex, so we omit the proofs.

Proposition 2.3 (Weinstein principle [59]) Let $\Omega$ be a relatively compact open set in $\mathbb{H}^{+}$, if $u: \Omega \rightarrow \mathbb{C}$ is $C^{2}$, then for all $m \in \mathbb{C}$,

$$
L_{m} u=x^{1-m} L_{2-m}\left[x^{m-1} u\right]
$$

Proposition 2.4 ([54]) Let $\Omega$ be a relatively compact open set in $\mathbb{H}^{+}$. If $u \in C^{2}(\Omega)$ and $m \in \mathbb{N}$, then $\Delta_{m+2}\left(T_{m} u\right)=T_{m}\left(L_{m} u\right)$.

These properties will allow us to calculate fundamental solutions for $L_{m}$ and $L_{m}^{\star}$ for $m \in \mathbb{N}$, and, thereafter, for $m \in \mathbb{Z}$. Finally, estimates of formulas for $L_{m}, L_{m}^{\star}, m \in \mathbb{Z}$, will show that these formulas actually provide fundamental solutions for $L_{m}$ and $L_{m}^{\star}$ in the case $m \in \mathbb{C}$.

\section{Integral expressions of fundamental solutions for integer values of $m$}

Let us recall the definition of the Dirac mass in a point $(x, y) \in \mathbb{R}^{2}$ :

$$
\left\langle\delta_{(x, y)}, \varphi\right\rangle=\varphi(x, y), \quad \varphi \in \mathcal{D}\left(\mathbb{R}^{2}\right) .
$$

Proposition 3.1 (partially in $[20,53,54]$ ) Let $m \in \mathbb{N}^{\star}$. For $(x, y) \in \mathbb{H}^{+}$and $(\xi, \eta) \in$ $\mathbb{H}^{+}$,

$$
E_{m}(x, y, \xi, \eta)=-\frac{\xi^{m}}{2 \pi} \int_{0}^{\pi} \frac{\sin ^{m-1} \theta d \theta}{\left[(x-\xi)^{2}+4 x \xi \sin ^{2} \theta / 2+(y-\eta)^{2}\right]^{m / 2}}
$$

is a fundamental solution on $\mathbb{H}^{+}$for the operator $L_{m, \xi, \eta}^{\star}$ at the fixed point $(x, y) \in \mathbb{H}^{+}$, which means

$$
L_{m, \xi, \eta}^{\star} E_{m}(x, y, \xi, \eta)=\delta_{(x, y)}(\xi, \eta)
$$

in the sense of distributions. Moreover, if $(\xi, \eta) \in \mathbb{H}^{+}$is fixed, then

$$
L_{m, x, y} E_{m}(x, y, \xi, \eta)=\delta_{(\xi, \eta)}(x, y)
$$

in the sense of distributions, which means that $E_{m}$ is a fundamental solution on $\mathbb{H}^{+}$ for the operator $L_{m, x, y}$ at the fixed point $(\xi, \eta) \in \mathbb{H}^{+}$. 
Proof Let $m \in \mathbb{N}^{*}$. Recall that

$$
E(x)=-\frac{1}{m \omega_{m+2}\|x\|^{m}}, \quad x \in \mathbb{R}^{m+2},
$$

is a fundamental solution for the Laplacian on $\mathbb{R}^{m+2}$, i.e. in the sense of distributions $\Delta_{m+2} E=\delta_{0}$, where $\omega_{m+2}$ is the area of the unit sphere in $\mathbb{R}^{m+2}$. Thus, for all $v \in \mathcal{D}\left(\mathbb{R}^{m+2}\right)$,

$$
v\left(t_{1}, \ldots, t_{m+2}\right)=-\frac{1}{m \omega_{m+2}} \int_{\mathbb{R}^{m+2}} \frac{\Delta_{m+2} v(\tau) d \tau_{1} d \tau_{2} \ldots d \tau_{m+2}}{\left(\left(\tau_{1}-t_{1}\right)^{2}+\cdots+\left(\tau_{m+2}-t_{m+2}\right)^{2}\right)^{m / 2}},
$$

where $\tau=\left(\tau_{1}, \ldots, \tau_{m+2}\right)$.

Applying this relation to the function $v=T_{m} u$, where $u \in \mathcal{D}\left(\mathbb{H}^{+}\right)$, and, due to Proposition 2.4 , for all $(x, y) \in \mathbb{H}^{+}$we have

$$
u(x, y)=-\frac{1}{m \omega_{m+2}} \int_{\mathbb{R}^{m+2}} \frac{\left(L_{m} u\right)\left(\sqrt{\xi_{1}^{2}+\cdots+\xi_{m+1}^{2}}, \xi_{m+2}\right) d \xi_{1} \ldots d \xi_{m+2}}{\left(\left(\xi_{1}-x\right)^{2}+\xi_{2}^{2}+\cdots+\xi_{m+1}^{2}+\left(\xi_{m+2}-y\right)^{2}\right)^{m / 2}}
$$

We will simplify this integral expression. For this, we will consider the following hyper-spherical coordinates:

$$
\begin{aligned}
\xi_{1} & =\xi \cos \theta_{1}, \\
\xi_{2} & =\xi \sin \theta_{1} \cos \theta_{2}, \\
& \ldots \\
\xi_{m-1} & =\xi \sin \theta_{1} \cdots \sin \theta_{m-2} \cos \theta_{m-1}, \\
\xi_{m} & =\xi \sin \theta_{1} \cdots \sin \theta_{m-1} \cos \theta_{m}, \\
\xi_{m+1} & =\xi \sin \theta_{1} \cdots \sin \theta_{m},
\end{aligned}
$$

where $\xi=\sqrt{\xi_{1}^{2}+\cdots+\xi_{m+1}^{2}} \geq 0, \theta_{m} \in(-\pi, \pi)$ and $\theta_{1}, \ldots, \theta_{m-1} \in(0, \pi)$. The absolute value of the determinant of the Jacobian matrix defined by this system of coordinates is

$$
\xi^{m} \sin \theta_{m-1} \sin ^{2} \theta_{m-2} \cdots \sin ^{m-1} \theta_{1} .
$$

Then, for all $(x, y) \in \mathbb{H}^{+}$,

$$
u(x, y)=\int_{-\infty}^{\infty} d \eta \int_{0}^{\infty} L_{m}(u)(\xi, \eta) E_{m}(x, y, \xi, \eta) d \xi
$$


with

$$
\begin{aligned}
& E_{m}(x, y, \xi, \eta) \\
& =-\frac{\xi^{m}}{m \omega_{m+2}} \int_{-\pi}^{\pi} d \theta_{m} \int_{\theta_{1}, \ldots, \theta_{m-1}=0}^{\pi} \frac{\sin \theta_{m-1} \sin ^{2} \theta_{m-2} \cdots \sin ^{m-1} \theta_{1} d \theta_{1} \ldots d \theta_{m-1}}{\left(\xi^{2}-2 x \xi \cos \theta_{1}+x^{2}+(y-\eta)^{2}\right)^{m / 2}}
\end{aligned}
$$

Let

$$
\begin{aligned}
\omega_{m} & =\int_{-\pi}^{\pi} d \theta_{m-1} \int_{\theta_{1}, \ldots, \theta_{m-2}=0}^{\pi} \sin \theta_{m-2} \sin ^{2} \theta_{m-3} \cdots \sin ^{m-2} \theta_{1} d \theta_{2} \ldots d \theta_{m-2} \\
& =\int_{\mathbb{S}_{m}} 1 d \sigma=\frac{2 \pi^{m / 2}}{\Gamma(m / 2)}
\end{aligned}
$$

then $E_{m}$ can be written as

$$
\begin{aligned}
E_{m}(x, y, \xi, \eta) & =-\frac{\omega_{m} \xi^{m}}{m \omega_{m+2}} \int_{0}^{\pi} \frac{\sin ^{m-1} \theta d \theta}{\left(\xi^{2}-2 x \xi \cos \theta+x^{2}+(y-\eta)^{2}\right)^{m / 2}} \\
& =-\frac{\xi^{m}}{2 \pi} \int_{0}^{\pi} \frac{\sin ^{m-1} \theta d \theta}{\left((x-\xi)^{2}+4 x \xi \sin ^{2} \theta / 2+(y-\eta)^{2}\right)^{m / 2}}
\end{aligned}
$$

Also, due to (2) we have

$$
L_{m, \xi, \eta}^{*} E_{m}(x, y, \xi, \eta)=\delta_{(x, y)}(\xi, \eta)
$$

Moreover, since for all $(x, y),(\xi, \eta) \in \mathbb{H}^{+}$,

$$
E_{m}(x, y, \xi, \eta)=\left(\frac{x}{\xi}\right)^{-m} E_{m}(\xi, \eta, x, y),
$$

and by Proposition 2.1, $S_{m}$ conjugates $L_{m}^{\star}$ and $L_{m}$, we have

$$
\begin{aligned}
L_{m, x, y} E_{m}(x, y, \xi, \eta) & =L_{m, x, y}\left(\left(\frac{x}{\xi}\right)^{-m} E_{m}(\xi, \eta, x, y)\right) \\
& =\left(\frac{x}{\xi}\right)^{-m} L_{m, x, y}^{\star} E_{m}(\xi, \eta, x, y)
\end{aligned}
$$

in the sense of distributions. Hence

$$
L_{m, x, y} E_{m}(x, y, \xi, \eta)=\left(\frac{x}{\xi}\right)^{-m} \delta_{(\xi, \eta)}(x, y)=\delta_{(\xi, \eta)},
$$

and the proof is complete.

This proposition and the Weinstein principle imply the following result. 
Proposition 3.2 (partially in $[20,53,54]$ ) Let $m \in \mathbb{Z} \backslash \mathbb{N}^{*}$. For $(x, y),(\xi, \eta) \in \mathbb{H}^{+}$,

$$
\begin{aligned}
E_{m}(x, y, \xi, \eta) & =\left(\frac{\xi}{x}\right)^{m-1} E_{2-m}(x, y, \xi, \eta) \\
& =-\frac{\xi x^{1-m}}{2 \pi} \int_{0}^{\pi} \frac{\sin ^{1-m} \theta d \theta}{\left[(x-\xi)^{2}+4 x \xi \sin ^{2} \theta / 2+(y-\eta)^{2}\right]^{1-m / 2}}
\end{aligned}
$$

is a fundamental solution on $\mathbb{H}^{+}$for the operator $L_{m, \xi, \eta}^{\star}$ at the fixed point $(x, y) \in \mathbb{H}^{+}$ and it is also a fundamental solution on $\mathbb{H}^{+}$for the operator $L_{m, x, y}$ at the fixed point $(\xi, \eta) \in \mathbb{H}^{+}$.

Proof For all $m \in \mathbb{N}^{*}, u \in \mathcal{D}\left(\mathbb{H}^{+}\right)$and $(x, y) \in \mathbb{H}^{+}$we have

$$
u(x, y)=\int_{(\xi, \eta) \in \mathbb{H}^{+}}\left(L_{m} u\right) E_{m}(x, y, \xi, \eta) d \xi d \eta,
$$

and by the Weinstein principle, Proposition 2.3, we have

$$
u(x, y)=\int_{\mathbb{H}^{+}} \xi^{1-m} L_{2-m}\left(\xi^{m-1} u\right) E_{m}(x, y, \xi, \eta) d \xi d \eta
$$

Denoting $v(x, y)=x^{m-1} u(x, y)$, we obtain

$$
x^{1-m} v(x, y)=\int_{\mathbb{H}^{+}} \xi^{1-m}\left(L_{2-m} v\right) E_{m}(x, y, \xi, \eta) d \xi d \eta
$$

then, for all $m^{\prime} \in \mathbb{Z} \backslash \mathbb{N}^{*}, v \in \mathcal{D}\left(\mathbb{H}^{+}\right)$and $(x, y) \in \mathbb{H}^{+}$, putting $m=2-m^{\prime}$, we have

$$
v(x, y)=\int_{\mathbb{H}^{+}}\left(L_{m^{\prime}} v\right)\left(\frac{\xi}{x}\right)^{m^{\prime}-1} E_{2-m^{\prime}}(x, y, \xi, \eta) d \xi d \eta
$$

The proof of the second statement is similar.

\section{Fundamental solutions for the Weinstein equation with complex coefficients}

In this section, we will generalize the result obtained in the previous section for $m \in \mathbb{Z}$ to the case $m \in \mathbb{C}$.

Let $m \in \mathbb{C}$. If $\operatorname{Re} m \geq 1$ put

$$
E_{m}=-\frac{\xi^{m}}{2 \pi} \int_{0}^{\pi} \frac{\sin ^{m-1} \theta d \theta}{\left[(x-\xi)^{2}+4 x \xi \sin ^{2} \theta / 2+(y-\eta)^{2}\right]^{m / 2}},
$$


and if $\operatorname{Re} m<1$ put

$$
E_{m}=-\frac{\xi x^{1-m}}{2 \pi} \int_{0}^{\pi} \frac{\sin ^{1-m} \theta d \theta}{\left[(x-\xi)^{2}+4 x \xi \sin ^{2} \theta / 2+(y-\eta)^{2}\right]^{1-m / 2}}
$$

here, if $\alpha>0$ is a real number and $\mu$ is a complex number, $\alpha^{\mu}=\exp (\mu \ln \alpha)$. Both values are well defined as the integrals on the right-hand side converge in the Lebesgue sense.

Proposition 4.1 For $m \in \mathbb{C}$ and $(\xi, \eta) \in \mathbb{H}^{+}$fixed, we have

$$
L_{m, x, y} E_{m}(x, y, \xi, \eta)=0, \quad(x, y) \in \mathbb{H}^{+} \backslash\{(\xi, \eta)\},
$$

and for $(x, y) \in \mathbb{H}^{+}$fixed, we have

$$
L_{m, \xi, \eta}^{\star} E_{m}(x, y, \xi, \eta)=0, \quad(\xi, \eta) \in \mathbb{H}^{+} \backslash\{(x, y)\}
$$

Proof For convenience, denote

$$
f_{m}(x, y, \xi, \eta, \theta)=\frac{1}{\left[(x-\xi)^{2}+4 x \xi \sin ^{2} \theta / 2+(y-\eta)^{2}\right]^{m / 2}} .
$$

To prove the first equality of the proposition, it suffices to show that

$$
\int_{0}^{\pi} L_{m, x, y} f_{m}(x, y, \xi, \eta, \theta) \sin ^{m-1} \theta d \theta=0 .
$$

Let us compute the partial derivatives of the function $f_{m}$ :

$$
\begin{aligned}
\partial_{x} f_{m}= & \frac{-m}{2} \frac{2(x-\xi)+4 \xi \sin ^{2} \theta / 2}{\left[(x-\xi)^{2}+4 x \xi \sin ^{2} \theta / 2+(y-\eta)^{2}\right]^{m / 2+1}} \\
\partial_{x x} f_{m}= & \frac{-m \quad\left(=-m(x-\xi \cos \theta) f_{m+2}\right),}{\left[(x-\xi)^{2}+4 x \xi \sin ^{2} \theta / 2+(y-\eta)^{2}\right]^{m / 2+1}} \\
& \quad+\frac{m}{2}\left(\frac{m}{2}+1\right) \frac{\left(2(x-\xi)+4 \xi \sin ^{2} \theta / 2\right)^{2}}{\left[(x-\xi)^{2}+4 x \xi \sin ^{2} \theta / 2+(y-\eta)^{2}\right]^{m / 2+2}}, \\
\partial_{y y} f_{m}= & \frac{-m}{\left[(x-\xi)^{2}+4 x \xi \sin ^{2} \theta / 2+(y-\eta)^{2}\right]^{m / 2+1}} \\
& +\frac{m}{2}\left(\frac{m}{2}+1\right) \frac{4(y-\eta)^{2}}{\left[(x-\xi)^{2}+4 x \xi \sin ^{2} \theta / 2+(y-\eta)^{2}\right]^{m / 2+2}} .
\end{aligned}
$$


We then have

$$
\begin{aligned}
\Delta f_{m}= & \frac{-2 m}{\left[(x-\xi)^{2}+4 x \xi \sin ^{2} \theta / 2+(y-\eta)^{2}\right]^{m / 2+1}} \\
& \quad+\frac{m}{2}\left(\frac{m}{2}+1\right) \frac{\left(2(x-\xi)+4 \xi \sin ^{2} \theta / 2\right)^{2}+4(y-\eta)^{2}}{\left[(x-\xi)^{2}+4 x \xi \sin ^{2} \theta / 2+(y-\eta)^{2}\right]^{m / 2+2}}
\end{aligned}
$$

Note that

$$
\begin{aligned}
\left(2(x-\xi)+4 \xi \sin ^{2} \frac{\theta}{2}\right)^{2} & +(2(y-\eta))^{2} \\
& =4\left[(x-\xi)^{2}+4 x \xi \sin ^{2} \frac{\theta}{2}+(y-\eta)^{2}\right]-4 \xi^{2} \sin ^{2} \theta
\end{aligned}
$$

hence

$$
\begin{aligned}
\Delta f_{m}= & \frac{m^{2}}{\left[(x-\xi)^{2}+4 x \xi \sin ^{2} \theta / 2+(y-\eta)^{2}\right]^{m / 2+1}} \\
& -\frac{m(m+2) \xi^{2} \sin ^{2} \theta}{\left[(x-\xi)^{2}+4 x \xi \sin ^{2} \theta / 2+(y-\eta)^{2}\right]^{m / 2+2}}
\end{aligned}
$$

Noting that

$$
\frac{\partial f_{m+2}}{\partial \theta}=-(m+2) \frac{x \xi \sin \theta}{\left[(x-\xi)^{2}+4 x \xi \sin ^{2} \theta / 2+(y-\eta)^{2}\right]^{m / 2+2}},
$$

we have

$$
\Delta f_{m}=m^{2} f_{m+2}+m \frac{\xi}{x} \sin \theta \frac{\partial f_{m+2}}{\partial \theta} .
$$

Integrating by parts, we have

$$
\begin{aligned}
\int_{0}^{\pi} \Delta f_{m} \sin ^{m-1} \theta d \theta & =m^{2} \int_{0}^{\pi} f_{m+2} \sin ^{m-1} \theta d \theta+m \frac{\xi}{x} \int_{0}^{\pi} \frac{\partial f_{m+2}}{\partial \theta} \sin ^{m} \theta d \theta \\
& =\frac{m}{x} \int_{0}^{\pi} m(x-\xi \cos \theta) f_{m+2} \sin ^{m-1} \theta d \theta \\
& =-\frac{m}{x} \int_{0}^{\pi} \partial_{x} f_{m} \sin ^{m-1} \theta d \theta
\end{aligned}
$$

and the result is deduced in the case $\operatorname{Re} m \geq 1$. The same argument is valid for $\operatorname{Re} m<1$. The second equality of the proposition can be deduced immediately from the fact that $S_{m}$ conjugates $L_{m}^{\star}$ and $L_{m}$, see Proposition 2.1 . 
In the sequel, we will denote

$$
d^{2}=(x-\xi)^{2}+(y-\eta)^{2}, \quad k=\frac{4 x \xi}{d^{2}}
$$

The following proposition describes the behavior of $E_{m}$ defined by (3) and (4) near its singularity. In particular, we show that the behavior of $E_{m}$ is close to the behavior of fundamental solutions for the Laplacian. This fact is well known for elliptic operators. But we emphasize here that in our proof the estimates of elliptic integrals are elementary (obtained using the dominated convergence theorem) and we do not use estimates arising from classical estimates of hypergeometric functions.

Proposition 4.2 Let $m \in \mathbb{C}$. For $(x, y) \in \mathbb{H}^{+}$fixed,

$$
E_{m}(x, y, \xi, \eta) \sim \frac{1}{2 \pi} \ln \sqrt{(x-\xi)^{2}+(y-\eta)^{2}} \quad \text { as } \quad(\xi, \eta) \rightarrow(x, y) .
$$

Proof We start with $\operatorname{Re} m \geq 1$. In this case, we have

$$
\begin{aligned}
E_{m}(x, y, \xi, \eta) & =-\frac{\xi^{m}}{2 \pi} \int_{0}^{\pi} \frac{\sin ^{m-1} \theta d \theta}{\left[(x-\xi)^{2}+4 x \xi \sin ^{2} \theta / 2+(y-\eta)^{2}\right]^{m / 2}} \\
& =-\frac{1}{2 \pi}\left(\frac{\xi}{d}\right)^{m} \int_{0}^{\pi} \frac{\sin ^{m-1} \theta d \theta}{\left(1+k \sin ^{2} \theta / 2\right)^{m / 2}} .
\end{aligned}
$$

Note that when $d \rightarrow 0, k$ tends to $+\infty$.

\section{Claim 4.3}

$$
\int_{0}^{\pi} \frac{\sin ^{m-1} \theta d \theta}{\left(1+k \sin ^{2} \theta / 2\right)^{m / 2}} \sim \frac{2^{m-1}}{k^{m / 2}} \ln k \quad \text { as } \quad k \rightarrow+\infty
$$

Proof Putting $u=\sin \theta / 2$, we have

$$
\begin{aligned}
\int_{0}^{\pi} \frac{\sin ^{m-1} \theta d \theta}{\left(1+k \sin ^{2} \theta / 2\right)^{m / 2}} & =2^{m} \int_{0}^{1} \frac{u^{m-1}\left(1-u^{2}\right)^{(m-2) / 2} d u}{\left(1+k u^{2}\right)^{m / 2}} \\
& =\frac{2^{m}}{k^{m / 2}} \int_{0}^{1} \frac{u^{m-1}\left(1-u^{2}\right)^{(m-2) / 2} d u}{\left(1 / k+u^{2}\right)^{m / 2}} .
\end{aligned}
$$

Note that

$$
\begin{aligned}
\int_{0}^{1} \frac{u^{m-1}\left(1-u^{2}\right)^{(m-2) / 2} d u}{\left(1 / k+u^{2}\right)^{m / 2}} & -\int_{0}^{1} \frac{u^{m-1} d u}{\left(1 / k+u^{2}\right)^{m / 2}} \\
& =-\int_{0}^{1} \frac{u^{m-1}}{\left(1 / k+u^{2}\right)^{m / 2}}\left(1-\left(1-u^{2}\right)^{(m-2) / 2}\right) d u
\end{aligned}
$$


where, due to monotone convergence, the right-hand side tends to

$$
-\int_{0}^{1} \frac{u^{m-1}}{\left(u^{2}\right)^{m / 2}}\left(1-\left(1-u^{2}\right)^{(m-2) / 2}\right) d u=-\int_{0}^{1} \frac{1-\left(1-u^{2}\right)^{(m-2) / 2}}{u} d u
$$

as $k \rightarrow+\infty$. The change of variable $u=\operatorname{sh} t / \sqrt{k}$ gives us

$$
\int_{0}^{1} \frac{u^{m-1} d u}{\left(1 / k+u^{2}\right)^{m / 2}}=\int_{0}^{\operatorname{argsh} \sqrt{k}} \operatorname{th}^{m-1} t d t
$$

Since $\mathrm{th}^{m-1} t$ tends to 1 as $t \rightarrow+\infty$, we deduce that as $k \rightarrow+\infty$,

$$
\int_{0}^{\operatorname{argsh} \sqrt{k}} \operatorname{th}^{m-1} d t \sim \int_{0}^{\operatorname{argsh} \sqrt{k}} d t=\operatorname{argsh} \sqrt{k} \sim \frac{1}{2} \ln k .
$$

The proof is complete.

Due to Claim 4.3, we have

$$
E_{m}(x, y, \xi, \eta) \sim-\frac{1}{2 \pi}\left(\frac{x}{d}\right)^{m} \frac{2^{m-1}}{k^{m / 2}} \ln k \sim \frac{1}{2 \pi} \ln d
$$

as $d \rightarrow 0+$. The case $\operatorname{Re} m<1$ is analogous.

Now, we can prove the main result of this section which shows that $E_{m}$ are fundamental solutions not only for $m \in \mathbb{N}$ but for all $m \in \mathbb{C}$.

Theorem 4.4 Let $m \in \mathbb{C}$. For $(x, y),(\xi, \eta) \in \mathbb{H}^{+}, E_{m}$ defined by (3) and (4) is a fundamental solution on $\mathbb{H}^{+}$for the operator $L_{m, \xi, \eta}^{\star}$ at the fixed point $(x, y) \in \mathbb{H}^{+}$, which means that on $\mathbb{H}^{+}$

$$
L_{m, \xi, \eta}^{\star} E_{m}(x, y, \xi, \eta)=\delta_{(x, y)}(\xi, \eta)
$$

in the sense of distributions. Moreover, if $(\xi, \eta) \in \mathbb{H}^{+}$is fixed, then on $\mathbb{H}^{+}$

$$
L_{m, x, y} E_{m}(x, y, \xi, \eta)=\delta_{(\xi, \eta)}(x, y)
$$

in the sense of distributions, which means that $E_{m}$ is a fundamental solution on $\mathbb{H}^{+}$ for the operator $L_{m, x, y}$ at the fixed point $(\xi, \eta) \in \mathbb{H}^{+}$.

Proof Let $m \in \mathbb{C}$ and $u \in \mathcal{D}\left(\mathbb{H}^{+}\right)$. Let $(x, y) \in \mathbb{H}^{+}$and $\varepsilon>0$ be such that $D((x, y), \varepsilon) \subset \mathbb{H}^{+}$, where $D((x, y), \varepsilon)$ is a disk with center $(x, y)$ and radius $\varepsilon$. Put

$$
\begin{aligned}
\mathrm{I}_{\varepsilon} & =\int_{\mathbb{H}^{+} \backslash D((x, y), \varepsilon)} L_{m}(u)(\xi, \eta) E_{m}(x, y, \xi, \eta) d \xi d \eta \\
& =\int_{\mathbb{H}^{+} \backslash D((x, y), \varepsilon)}\left(L_{m}(u)(\xi, \eta) E_{m}(x, y, \xi, \eta)-u(\xi, \eta) L_{m}^{\star}\left(E_{m}\right)(x, y, \xi, \eta)\right) d \xi d \eta
\end{aligned}
$$


We use the fact that $L_{m}^{\star}\left(E_{m}\right)=0$ on $\mathbb{H}^{+} \backslash D((x, y), \varepsilon)$. An elementary computation gives

$$
\begin{aligned}
L_{m}(u) E_{m}-u L_{m}^{\star}(E)=\partial_{\xi}\left(\left(\partial_{\xi} u\right) E_{m}\right. & \left.-u\left(\partial_{\xi} E_{m}\right)+\frac{m}{\xi} u E_{m}\right) \\
& +\partial_{\eta}\left(\left(\partial_{\eta} u\right) E_{m}-u\left(\partial_{\eta} E_{m}\right)\right) .
\end{aligned}
$$

Let us recall the Green formula: If $\Omega$ is an open set in $\mathbb{R}^{2}$ with a piecewise $C^{1}$ differentiable boundary, then

$$
\int_{\Omega} \operatorname{div} X(x, y) d x d y=\int_{\partial \Omega} X(x, y) \cdot \mathbf{n}(x, y) d s,
$$

where $\mathbf{n}$ is the outer unit normal vector to $\partial \Omega$ and $d s$ the arc length element on $\partial \Omega$ (positively oriented), $X=\left(X_{1}, X_{2}\right): \bar{\Omega} \rightarrow \mathbb{C}^{2}$ is a $C^{1}$-vector field.

Applying this formula to the open set $\Omega=U \backslash D((x, y), \varepsilon)$, where $U$ is a regular open set in $\mathbb{H}^{+}$containing the support of $u$, we have

$$
\begin{aligned}
& \mathrm{I}_{\varepsilon}=-\int_{\substack{t \in[0,2 \pi] \\
(\xi, \eta)=(x, y)+\varepsilon(\cos t, \sin t)}}\left(\left(\left(\partial_{\xi} u\right) E_{m}-u\left(\partial_{\xi} E_{m}\right)+\frac{m}{\xi} u E_{m}\right) \cos t\right. \\
& \left.+\left(\left(\partial_{\eta} u\right) E_{m}-u\left(\partial_{\eta} E_{m}\right)\right) \sin t\right) \varepsilon d t .
\end{aligned}
$$

Proposition 4.2 implies

$$
\int_{\substack{t \in[0,2 \pi] \\(\xi, \eta)=(x, y)+\varepsilon(\cos t, \sin t)}}\left(\left(\left(\partial_{\xi} u\right)+\frac{m}{\xi} u\right) \cos t+\left(\partial_{\eta} u\right) \sin t\right) E_{m} \varepsilon d t \rightarrow 0
$$

as $\varepsilon \rightarrow 0+$ because $\lim _{\varepsilon \rightarrow 0} \varepsilon \ln \varepsilon=0$. Then, if we want to prove that $\lim _{\varepsilon \rightarrow 0} \mathrm{I}_{\varepsilon}$ exists, we have to prove the existence of

$$
\lim _{\varepsilon \rightarrow 0} \int_{\substack{t \in[0,2 \pi] \\(\xi, \eta)=(x, y)+\varepsilon(\cos t, \sin t)}} u\left(\left(\partial_{\xi} E_{m}\right) \cos t+\left(\partial_{\eta} E_{m}\right) \sin t\right) \varepsilon d t
$$

and this limit will be equal to the limit of $\mathrm{I}_{\varepsilon}$. 
Now, assume that $\operatorname{Re} m \geq 1$. Denote by $\mathrm{J}_{\varepsilon}$ the integral in the previous expression. A computation gives

$$
\begin{aligned}
& \mathbf{J}_{\varepsilon}=-\underbrace{\frac{m}{2 \pi} \int_{\substack{t \in[0,2 \pi] \\
(\xi, \eta)=(x, y)+\varepsilon(\cos t, \sin t)}} u \frac{\xi^{m-1}}{\varepsilon^{m}} \int_{0}^{\pi} \frac{\sin ^{m-1} \theta d \theta}{\left(1+k \sin ^{2} \theta / 2\right)^{m / 2}} \varepsilon \cos t d t}_{\mathbf{J}_{\varepsilon, 1}} \\
& +\underbrace{\frac{m}{2 \pi} \int_{\substack{t \in[0,2 \pi] \\
(\xi, \eta)=(x, y)+\varepsilon(\cos t, \sin t)}} u \frac{\xi^{m}}{\varepsilon^{m+2}} \int_{0}^{\pi} \frac{\sin ^{m-1} \theta d \theta}{\left(1+k \sin ^{2} \theta / 2\right)^{m / 2+1}} \varepsilon^{2} d t}_{\mathrm{J}_{\varepsilon, 2}} \\
& +\underbrace{\frac{m}{2 \pi} \int_{\substack{t \in[0,2 \pi] \\
(\xi, \eta)=(x, y)+\varepsilon(\cos t, \sin t)}} u \frac{\xi^{m}}{\varepsilon^{m+2}} \int_{0}^{\pi} \frac{2 x \sin ^{2} \theta / 2 \sin ^{m-1} \theta d \theta}{\left(1+k \sin ^{2} \theta / 2\right)^{m / 2+1}} \varepsilon \cos t d t}_{\mathbf{J}_{\varepsilon, 3}},
\end{aligned}
$$

where $k=4 x \xi / \varepsilon^{2}$.

\section{Claim 4.5}

$$
\int_{0}^{\pi} \frac{\sin ^{2} \theta / 2 \sin ^{m-1} \theta d \theta}{\left(1+k \sin ^{2} \theta / 2\right)^{m / 2+1}} \sim \frac{2^{m-1}}{k^{m / 2+1}} \ln k \quad \text { as } \quad k \rightarrow+\infty .
$$

Proof We put $u=\sin \theta / 2$, then

$$
\begin{aligned}
\int_{0}^{\pi} \frac{\sin ^{2} \theta / 2 \sin ^{m-1} \theta d \theta}{\left(1+k \sin ^{2} \theta / 2\right)^{m / 2+1}} & =2^{m} \int_{0}^{1} \frac{u^{m+1}\left(1-u^{2}\right)^{(m-2) / 2} d u}{\left(1+k u^{2}\right)^{m / 2+1}} \\
& =\frac{2^{m}}{k^{m / 2+1}} \int_{0}^{1} \frac{u^{m+1}\left(1-u^{2}\right)^{(m-2) / 2} d u}{\left(1 / k+u^{2}\right)^{m / 2+1}} .
\end{aligned}
$$

Note that

$$
\begin{aligned}
\int_{0}^{1} \frac{u^{m+1}\left(1-u^{2}\right)^{(m-2) / 2} d u}{\left(1 / k+u^{2}\right)^{m / 2+1}} & -\int_{0}^{1} \frac{u^{m+1} d u}{\left(1 / k+u^{2}\right)^{m / 2+1}} \\
& =-\int_{0}^{1} \frac{u^{m+1}}{\left(1 / k+u^{2}\right)^{m / 2+1}}\left(1-\left(1-u^{2}\right)^{(m-2) / 2}\right) d u \\
& \rightarrow-\int_{0}^{1} \frac{u^{m+1}}{\left(u^{2}\right)^{m / 2+1}}\left(1-\left(1-u^{2}\right)^{(m-2) / 2}\right) d u \\
& =-\int_{0}^{1} \frac{1-\left(1-u^{2}\right)^{(m-2) / 2}}{u} d u
\end{aligned}
$$


as $k \rightarrow+\infty$. The change of variable $u=\operatorname{sh} t / \sqrt{k}$ gives us

$$
\int_{0}^{1} \frac{u^{m+1} d u}{\left(1 / k+u^{2}\right)^{m / 2+1}}=\int_{0}^{\operatorname{argsh} \sqrt{k}} \operatorname{th}^{m+1} t d t .
$$

Since $\mathrm{th}^{m+1} t$ tends to 1 as $t \rightarrow+\infty$, it follows that as $k \rightarrow+\infty$

$$
\int_{0}^{\operatorname{argsh} \sqrt{k}} \operatorname{th}^{m+1} t d t \sim \int_{0}^{\operatorname{argsh} \sqrt{k}} d t=\operatorname{argsh} \sqrt{k} \sim \frac{1}{2} \ln k .
$$

\section{Claim 4.6}

$$
\int_{0}^{\pi} \frac{\sin ^{m-1} \theta d \theta}{\left(1+k \sin ^{2} \theta / 2\right)^{m / 2+1}} \sim \frac{2^{m}}{m k^{m / 2}} \quad \text { as } \quad k \rightarrow+\infty .
$$

Proof Putting as previously $u=\sin \theta / 2$, we have

$$
\begin{aligned}
\int_{0}^{\pi} \frac{\sin ^{m-1} \theta d \theta}{\left(1+k \sin ^{2} \theta / 2\right)^{m / 2+1}} & =2^{m} \int_{0}^{1} \frac{u^{m-1}\left(1-u^{2}\right)^{(m-2) / 2} d u}{\left(1+k u^{2}\right)^{m / 2+1}} \\
& =\frac{2^{m}}{k^{m / 2+1}} \int_{0}^{1} \frac{u^{m-1}\left(1-u^{2}\right)^{(m-2) / 2} d u}{\left(1 / k+u^{2}\right)^{m / 2+1}} .
\end{aligned}
$$

Note that

$$
\begin{aligned}
\int_{0}^{1} \frac{u^{m-1}\left(1-u^{2}\right)^{(m-2) / 2} d u}{\left(1 / k+u^{2}\right)^{m / 2+1}} & -\int_{0}^{1} \frac{u^{m-1} d u}{\left(1 / k+u^{2}\right)^{m / 2+1}} \\
& =-\int_{0}^{1} \frac{u^{m-1}\left(1-\left(1-u^{2}\right)^{(m-2) / 2}\right)}{\left(1 / k+u^{2}\right)^{m / 2+1}} d u
\end{aligned}
$$

Let us estimate the right-hand side of this equality:

$$
\begin{aligned}
\int_{0}^{1} & \frac{u^{m-1}\left(1-\left(1-u^{2}\right)^{(m-2) / 2}\right) d u}{\left(1 / k+u^{2}\right)^{m / 2+1}}-\int_{0}^{1} \frac{u^{m-1}}{\left(1 / k+u^{2}\right)^{m / 2+1}}\left(\frac{m-2}{2} u^{2}\right) d u \\
= & \int_{0}^{1} \frac{u^{m-1}}{\left(1 / k+u^{2}\right)^{m / 2+1}}\left(1-\frac{m-2}{2} u^{2}-\left(1-u^{2}\right)^{(m-2) / 2}\right) d u \\
& \rightarrow \int_{0}^{1} \frac{u^{m-1}}{\left(u^{2}\right)^{m / 2+1}}\left(1-\frac{m-2}{2} u^{2}-\left(1-u^{2}\right)^{(m-2) / 2}\right) d u \\
& =\int_{0}^{1} \frac{1-(m-2) u^{2} / 2-\left(1-u^{2}\right)^{(m-2) / 2}}{u^{3}} d u
\end{aligned}
$$

as $k \rightarrow+\infty$. As seen in the proof of Claim 4.5, we have

$$
\frac{m-2}{2} \int_{0}^{1} \frac{u^{m+1}}{\left(1 / k+u^{2}\right)^{m / 2+1}} d u \sim \frac{m-2}{4} \ln k
$$


as $k \rightarrow+\infty$. Due to $(\star)$ and $(\star \star)$, we have

$$
\int_{0}^{1} \frac{u^{m-1}}{\left(1 / k+u^{2}\right)^{m / 2+1}}\left(1-\left(1-u^{2}\right)^{(m-2) / 2}\right) d u \sim \frac{m-2}{4} \ln k
$$

as $k \rightarrow+\infty$. The change of variable $u=\operatorname{sh} t / \sqrt{k}$ gives

$$
\int_{0}^{1} \frac{u^{m-1} d u}{\left(1 / k+u^{2}\right)^{m / 2+1}}=k \int_{0}^{\operatorname{argsh} \sqrt{k}} \frac{\operatorname{th}^{m-1} t}{\operatorname{ch}^{2} t} d t=\frac{k}{m} \operatorname{th}^{m}(\operatorname{argsh} \sqrt{k}) .
$$

It follows that as $k \rightarrow+\infty$,

$$
\int_{0}^{1} \frac{u^{m-1} d u}{\left(1 / k+u^{2}\right)^{m / 2+1}} \sim \frac{k}{m}
$$

Thus

$$
\int_{0}^{1} \frac{u^{m-1}\left(1-u^{2}\right)^{(m-2) / 2} d u}{\left(1 / k+u^{2}\right)^{m / 2+1}} \sim \frac{k}{m}
$$

and

$$
\int_{0}^{\pi} \frac{\sin ^{m-1} \theta d \theta}{\left(1+k \sin ^{2} \theta / 2\right)^{m / 2+1}} \sim \frac{2^{m}}{m k^{m / 2}}
$$

as $k \rightarrow+\infty$. This completes the proof.

Let us return to the proof of Theorem 4.4. Claim 4.3 implies

$$
\begin{aligned}
\mathrm{J}_{\varepsilon, 1} & \sim-\frac{m}{2 \pi} \int_{\substack{t \in[0,2 \pi] \\
(\xi, \eta)=(x, y)+\varepsilon(\cos t, \sin t)}} u \frac{x^{m-1}}{\varepsilon^{m}} \frac{2^{m-1}}{k^{m / 2}} \ln k \varepsilon \cos t d t \\
& \sim+\frac{m}{2 \pi x} \varepsilon \ln \varepsilon \int_{\substack{t \in[0,2 \pi] \\
(\xi, \eta)=(x, y)+\varepsilon(\cos t, \sin t)}} u(x+\varepsilon \cos t, y+\varepsilon \sin t) \cos t d t
\end{aligned}
$$

which tends to 0 as $\varepsilon \rightarrow 0+$.

Claim 4.5 implies

$$
\begin{aligned}
\mathrm{J}_{\varepsilon, 3} & \sim \frac{m}{2 \pi} \int_{\substack{t \in[0,2 \pi] \\
(\xi, \eta)=(x, y)+\varepsilon(\cos t, \sin t)}} u \frac{x^{m}}{\varepsilon^{m+2}} 2 x \frac{2^{m-1}}{k^{m / 2+1}} \ln k \varepsilon \cos t d t \\
& \sim-\frac{m}{4 \pi x} \varepsilon \ln \varepsilon \int_{\substack{t \in[0,2 \pi] \\
(\xi, \eta)=(x, y)+\varepsilon(\cos t, \sin t)}} u(x+\varepsilon \cos t, y+\varepsilon \sin t) \cos t d t
\end{aligned}
$$

which tends to 0 as $\varepsilon \rightarrow 0+$. 
Finally, Claim 4.6 implies

$$
\begin{aligned}
\mathrm{J}_{\varepsilon, 2} & \sim \frac{m}{2 \pi} \int_{\substack{t \in[0,2 \pi] \\
(\xi, \eta)=(x, y)+\varepsilon(\cos t, \sin t)}} u \frac{x^{m}}{\varepsilon^{m+2}} \frac{2^{m}}{m k^{m / 2}} \varepsilon^{2} d t \\
& \sim \frac{1}{2 \pi} \int_{\substack{t \in[0,2 \pi] \\
(\xi, \eta)=(x, y)+\varepsilon(\cos t, \sin t)}} u(x+\varepsilon \cos t, y+\varepsilon \sin t) d t
\end{aligned}
$$

which tends to $u(x, y)$ as $\varepsilon \rightarrow 0+$.

So we have proved that for all $m \in \mathbb{C}$ such that $\operatorname{Re} m>0$,

$$
\begin{aligned}
\lim _{\varepsilon \rightarrow 0+} \int_{\mathbb{H}^{+} \backslash D((x, y), \varepsilon)} & L_{m}(u)(\xi, \eta) E_{m}(x, y, \xi, \eta) d \xi d \eta \\
& =\int_{\mathbb{H}^{+}} L_{m}(u)(\xi, \eta) E_{m}(x, y, \xi, \eta) d \xi d \eta=u(x, y)
\end{aligned}
$$

therefore $E_{m}$ indeed is a fundamental solution for the operator $L_{m}^{\star}$ for all $m \in \mathbb{C}$ with $\operatorname{Re} m>0$. The case $m \in \mathbb{C}$ with $\operatorname{Re} m \leq 1$ is similar.

Due to Proposition 2.1, we also have dual assertions for fundamental solutions for the operator $L_{m}$.

The following proposition is roughly a consequence of the previous theorem. Of course, it is a classical statement, but we would like to present its short proof.

Proposition 4.7 Let $m \in \mathbb{C}$ and let $\Omega$ be a relatively compact open set in $\mathbb{H}^{+}$whose boundary is piecewise $C^{1}$-differentiable. Then, for $(x, y) \in \Omega$ and $u \in C^{2}(\bar{\Omega})$, we have

$$
\begin{aligned}
u(x, y)= & \int_{\Omega} L_{m}(u) E_{m} d \xi d \eta \\
& -\int_{\partial \Omega}\left[\left(\partial_{\xi} u\right) E_{m}-u\left(\partial_{\xi} E_{m}\right)+\frac{m}{\xi} u E_{m},\left(\partial_{\eta} u\right) E_{m}-u\left(\partial_{\eta} E_{m}\right)\right] \cdot \mathbf{n} d s
\end{aligned}
$$

where $u=u(\xi, \eta), E_{m}=E_{m}(x, y, \xi, \eta), \mathbf{n}$ is the outer unit normal vector to $\partial \Omega$ and $d s$ is the arc length element on $\partial \Omega$ (positively oriented).

Proof Indeed, if $u \in C^{2}(\bar{\Omega})$, for $(x, y) \in \Omega$ and $\varepsilon>0$ such that $\overline{D((x, y), \varepsilon)} \subset \Omega$, we have

$$
\int_{\Omega \backslash D((x, y), \varepsilon)} L_{m}(u) E_{m} d \xi d \eta=\int_{\Omega \backslash D((x, y), \varepsilon)}\left(L_{m}(u) E_{m}-L_{m}^{\star}\left(E_{m}\right) u\right) d \xi d \eta
$$


By the Green formula, the latter integral is equal to

$$
\begin{aligned}
& \int_{\partial \Omega}\left[\left(\partial_{\xi} u\right) E_{m}-u\left(\partial_{\xi} E_{m}\right)+\right.\left.\frac{m}{\xi} u E_{m},\left(\partial_{\eta} u\right) E_{m}-u\left(\partial_{\eta} E_{m}\right)\right] \cdot \mathbf{n} d s \\
&-\int_{\substack{t \in[0, \eta)=(x, y)+\varepsilon(\cos t, \sin t) \\
(\xi, \eta)}}\left(\left(\left(\partial_{\xi} u\right) E_{m}-u\left(\partial_{\xi} E_{m}\right)+\frac{m}{\xi} u E_{m}\right) \cos t\right. \\
&\left.+\left(\left(\partial_{\eta} u\right) E_{m}-u\left(\partial_{\eta} E_{m}\right)\right) \sin t\right) \varepsilon d t
\end{aligned}
$$

and, as we saw in the previous proof, it tends to

$$
\int_{\partial \Omega}\left[\left(\partial_{\xi} u\right) E_{m}-u\left(\partial_{\xi} E_{m}\right)+\frac{m}{\xi} u E_{m},\left(\partial_{\eta} u\right) E_{m}-u\left(\partial_{\eta} E_{m}\right)\right] \cdot \mathbf{n} d s+u(x, y),
$$

as $\varepsilon \rightarrow 0$. Due to integrability of $E_{m}$ near $(x, y)$ we have

$$
\lim _{\varepsilon \rightarrow 0} \int_{\Omega \backslash D((x, y), \varepsilon)} L_{m}(u) E_{m} d \xi d \eta=\int_{\Omega} L_{m}(u) E_{m} d \xi d \eta,
$$

and the proof is complete.

\section{Liouville-type result and decomposition theorem for axisymmetric potentials}

In the previous section, we have seen that fundamental solutions $E_{m}$ in the complex case have different expressions depending on whether $\operatorname{Re} m<1$ or $\operatorname{Re} m \geq 1$. Hence the behavior of $E_{m}$ will be different in each case.

We will modify fundamental solutions so that they vanish at the boundary of $\mathbb{H}^{+}$, which means that they tend to zero on the $y$-axis and at infinity. Expression (4) satisfies this property: $E_{m}(x, y, \cdot, \cdot)$ tends to 0 as $x \rightarrow 0+$ and $\|(x, y)\| \rightarrow+\infty$; whereas (3) does not. Consider

$$
E_{m}(x, y, \xi, \eta)-E_{m}(-x, y, \xi, \eta), \quad \operatorname{Re} m \geq 1
$$

It is also a fundamental solution on $\mathbb{H}^{+}$and it satisfies the required property. Let us put

- for $\operatorname{Re} m<1$,

$$
F_{m}(x, y, \xi, \eta)=E_{m}(x, y, \xi, \eta)
$$

- for $\operatorname{Re} m \geq 1$,

$$
F_{m}(x, y, \xi, \eta)=E_{m}(x, y, \xi, \eta)-E_{m}(-x, y, \xi, \eta)
$$


We will need the following definition of convergence on the boundary of $\mathbb{H}^{+}$.

Definition 5.1 Let $u: \mathbb{H}^{+} \rightarrow \mathbb{R}$ be a function defined on $\mathbb{H}^{+}$. We write

$$
\lim _{\partial \mathbb{H}^{+}} u=0
$$

if and only if for all $\varepsilon>0$ there exists $N \in \mathbb{N}$ such that for all $n>N$ and all $(x, y) \in H^{+}, x \leq 1 / n$ or $\|(x, y)\| \geq n$ implies $|u(x, y)| \leq \varepsilon$.

Proposition 5.2 Let $u: \mathbb{H}^{+} \rightarrow \mathbb{C}$. We have $\lim _{\partial \mathbb{H}^{+}} u=0$ if and only if

$$
\lim _{\|(x, y)\| \rightarrow+\infty} u(x, y)=0 \quad \text { and } \quad \lim _{(0, y)} u=0, \quad y \in \mathbb{R}
$$

Proof The direct implication is easy. Conversely, assume $\lim _{\|(x, y)\| \rightarrow+\infty} u(x, y)=0$ and $\lim _{(0, y)} u=0, y \in \mathbb{R}$. Let $\varepsilon>0$, then there is $A>0$ such that for all $(\xi, \eta) \in \mathbb{H}^{+}$, $\sqrt{\xi^{2}+\eta^{2}} \geq A$ implies $|u(\xi, \eta)| \leq \varepsilon$. Similarly, for all $y \in \mathbb{R}$, there is $\alpha_{y} \in(0,1)$ such that for all $(\xi, \eta) \in \mathbb{H}^{+}, \sqrt{\xi^{2}+(\eta-y)^{2}}<\alpha_{y}$ implies $|u(\xi, \eta)| \leq \varepsilon$.

The interval $[-A, A]$ is compact. By the Lebesgue covering lemma, there is $\alpha>0$ such that for all $y^{\prime} \in[-A, A]$, the ball $B\left(y^{\prime}, \alpha\right)$ is included in one of the balls $B\left(y, \alpha_{y}\right)$ with $y \in[-A, A]$. In particular, if $(\xi, \eta) \in \mathbb{H}^{+}$is such that $0<\xi<\alpha$, then $|u(\xi, \eta)| \leq \varepsilon$. This completes the proof.

The following proposition is a Liouville-type result for axisymmetric potentials in the right half-plane. As we mentioned in the introduction, this result is not trivial due to the loss of strict ellipticity of the Weinstein operator on the $y$-axis. Let us mention that in [5, Theorem 7.1] one can find an interesting result on the description of a class of non-strictly elliptic equations with unbounded coefficients.

Proposition 5.3 Let $u \in C^{2}\left(\mathbb{H}^{+}\right)$be such that $L_{m} u=0$ and $\lim _{\partial \mathbb{H}^{+}} u=0$. Then $u \equiv 0$ on $\mathbb{H}^{+}$.

Proof For $(\xi, \eta) \in \mathbb{H}^{+}$and $N \in \mathbb{N}^{*}$, define

$$
\phi_{N}(\xi, \eta)=\theta_{1}(N \xi) \theta_{2}\left(\frac{\xi}{N}\right) \theta_{2}\left(\frac{\eta}{N}\right),
$$

where $\theta_{1}$ and $\theta_{2}$ are smooth functions on $\mathbb{R}$, valued on $[0,1]$ and such that $\theta_{1}(t)=1$ for $t \geq 1, \theta_{1}(t)=0$ for $t \leq 1 / 2, \theta_{2}(t)=1$ for $t \in[-1 / 2,1 / 2]$, and $\theta_{2}(t)=0$ for $t \in \mathbb{R} \backslash(-1,1)$. Assume also that all derivatives of $\theta_{1}$ and $\theta_{2}$ vanish at $\{-1,-1 / 2$, $1 / 2,1\}$ (Fig. 2).

If $u \in C^{2}\left(\mathbb{H}^{+}\right)$satisfies $L_{m} u=0$, then $u \phi_{N} \in C^{2}\left(\mathbb{H}^{+}\right)$and it is compactly supported on $\mathbb{H}^{+}$. Throughout the following, we fix $(x, y) \in \mathbb{H}^{+}$. For $N$ sufficiently large, due to Proposition 4.7 (true if $E_{m}$ is replaced by $F_{m}$ ), we have

$$
u(x, y)=u(x, y) \phi_{N}(x, y)=\int_{\mathbb{H}^{+}} L_{m}\left(u \phi_{N}\right) F_{m} d \xi d \eta
$$



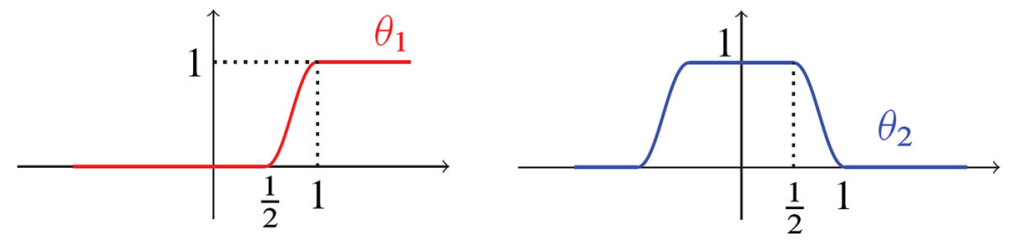

Fig. 2 The functions $\theta_{1}$ and $\theta_{2}$

(because the function $L_{m}\left(u \phi_{N}\right)$ is identically zero in a neighborhood of the singularity of $\left.F_{m}\right)$, thus

$$
\begin{aligned}
u(x, y) & =\int_{\mathbb{H}^{+}}\left[L_{m}(u) \phi_{N}+u L_{m}\left(\phi_{N}\right)+2 \nabla u \cdot \nabla \phi_{N}\right] F_{m} d \xi d \eta \\
& =\int_{\mathbb{H}^{+}} u\left[L_{m}\left(\phi_{N}\right) F_{m}-2 \operatorname{div}\left(F_{m} \nabla \phi_{N}\right)\right] d \xi d \eta \\
& =\int_{D_{1} \cup \ldots \cup D_{8}} u\left[L_{m}\left(\phi_{N}\right) F_{m}-2 \operatorname{div}\left(F_{m} \nabla \phi_{N}\right)\right] d \xi d \eta \\
& =-\int_{D_{1} \cup \cdots \cup D_{8}} u\left[L_{-m}\left(\phi_{N}\right) F_{m}+2 \nabla F_{m} \cdot \nabla \phi_{N}\right] d \xi d \eta,
\end{aligned}
$$

where $D_{1}, \ldots, D_{8}$ are the following domains (which depend on $N$ ) (Fig. 3):

$$
D_{1}=\left[\frac{1}{2 N}, \frac{1}{N}\right] \times\left[-\frac{N}{2}, \frac{N}{2}\right], \quad D_{2}=\left[\frac{1}{N}, \frac{N}{2}\right] \times\left[\frac{N}{2}, N\right]
$$

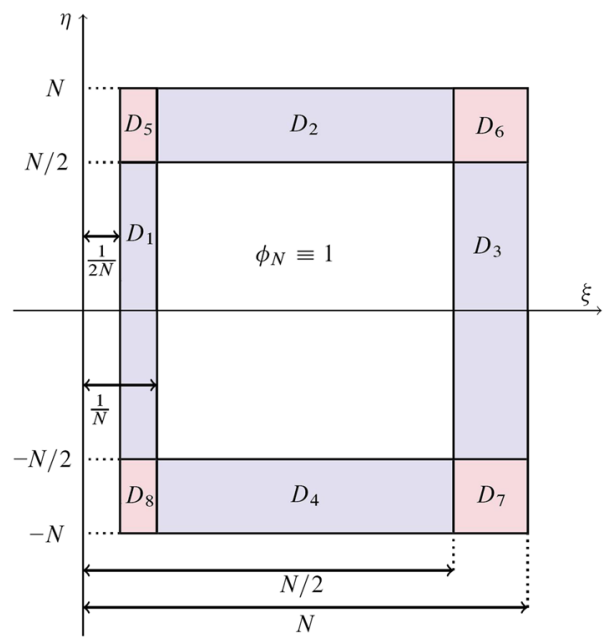

Fig. 3 Domains $D_{i}$ 


$$
\begin{aligned}
D_{3} & =\left[\frac{N}{2}, N\right] \times\left[-\frac{N}{2}, \frac{N}{2}\right], & D_{4} & =\left[\frac{1}{N}, \frac{N}{2}\right] \times\left[-N,-\frac{N}{2}\right], \\
D_{5} & =\left[\frac{1}{2 N}, \frac{1}{N}\right] \times\left[\frac{N}{2}, N\right], & D_{6} & =\left[\frac{N}{2}, N\right] \times\left[\frac{N}{2}, N\right], \\
D_{7} & =\left[\frac{N}{2}, N\right] \times\left[-N,-\frac{N}{2}\right], & D_{8} & =\left[\frac{1}{2 N}, \frac{1}{N}\right] \times\left[-N,-\frac{N}{2}\right] .
\end{aligned}
$$

Since $\lim _{\partial \mathbb{H}}+u=0$

$$
u_{N}=\sup _{(\xi, \eta) \in D_{1} \cup \ldots \cup D_{8}}|u(\xi, \eta)| \rightarrow 0 \quad \text { as } \quad N \rightarrow+\infty
$$

We will estimate integrals over sets $D_{1}, \ldots, D_{8}$ separately, see auxiliary lemmas below. Recall that, if $\left(u_{N}\right)_{N}$ and $\left(v_{N}\right)_{N}$ are complex sequences, $u_{N}=\mathrm{O}\left(v_{N}\right)$ means that there exists a constant $M$ such that, for every $N$ sufficiently large, $\left|u_{N}\right| \leq M\left|v_{N}\right|$; $u_{N}=\mathrm{o}\left(v_{N}\right)$ means that for every $\varepsilon>0$, for every $N$ sufficiently large, $\left|u_{N}\right| \leq \varepsilon\left|v_{N}\right|$.

Lemma 5.4 On $D_{1}$, we have

$$
\sup \left|\frac{\partial \phi_{N}}{\partial \xi}\right|=\mathrm{O}(N) \quad \text { and } \quad \sup \left|\frac{\partial \phi_{N}}{\partial \eta}\right|=0 \text {. }
$$

On $D_{2} \cup D_{4}$, we have

$$
\sup \left|\frac{\partial \phi_{N}}{\partial \xi}\right|=0 \quad \text { and } \quad \sup \left|\frac{\partial \phi_{N}}{\partial \eta}\right|=\mathrm{O}\left(\frac{1}{N}\right)
$$

On $D_{3}$, we have

$$
\sup \left|\frac{\partial \phi_{N}}{\partial \xi}\right|=\mathrm{O}\left(\frac{1}{N}\right) \quad \text { and } \quad \sup \left|\frac{\partial \phi_{N}}{\partial \eta}\right|=0
$$

On $D_{5} \cup D_{8}$, we have

$$
\sup \left|\frac{\partial \phi_{N}}{\partial \xi}\right|=\mathrm{O}(N) \quad \text { and } \quad \sup \left|\frac{\partial \phi_{N}}{\partial \eta}\right|=\mathrm{O}\left(\frac{1}{N}\right) \text {. }
$$

On $D_{6} \cup D_{7}$, we have

$$
\sup \left|\frac{\partial \phi_{N}}{\partial \xi}\right|=\mathrm{O}\left(\frac{1}{N}\right) \quad \text { and } \quad \sup \left|\frac{\partial \phi_{N}}{\partial \eta}\right|=\mathrm{O}\left(\frac{1}{N}\right) \text {. }
$$

On $D_{1} \cup D_{5} \cup D_{8}$, we have

$$
\sup \left|L_{-m}\left(\phi_{N}\right)\right|=\mathrm{O}\left(N^{2}\right) .
$$


On $D_{2} \cup D_{3} \cup D_{4} \cup D_{6} \cup D_{7}$, we have

$$
\sup \left|L_{-m}\left(\phi_{N}\right)\right|=\mathrm{O}\left(\frac{1}{N^{2}}\right)
$$

Proof For $(\xi, \eta) \in D_{1}, \phi_{N}(\xi, \eta)=\theta_{1}(N \xi)$ and thus

$$
\begin{aligned}
\frac{\partial \phi_{N}}{\partial \xi}(\xi, \eta) & =N \theta_{1}^{\prime}(N \xi), \quad \frac{\partial \phi_{N}}{\partial \eta}(\xi, \eta)=0, \\
L_{-m} \phi_{N}(\xi, \eta) & =N^{2} \theta_{1}^{\prime \prime}(N \xi)-\frac{m N}{\xi} \theta_{1}^{\prime}(N \xi),
\end{aligned}
$$

which give us

$$
\sup _{D_{1}}\left|\frac{\partial \phi_{N}}{\partial \xi}\right|=\mathrm{O}(N), \quad \sup _{D_{1}}\left|\frac{\partial \phi_{N}}{\partial \eta}\right|=0, \quad \sup _{D_{1}}\left|L_{-m}\left(\phi_{N}\right)\right|=\mathrm{O}\left(N^{2}\right)
$$

since the derivatives of $\theta_{1}$ are bounded and for $(\xi, \eta) \in D_{1}$ one gets $\xi \geq 1 /(2 N)$.

For $(\xi, \eta) \in D_{2}, \phi_{N}(\xi, \eta)=\theta_{2}(\eta / N)$ and thus

$$
\begin{gathered}
\frac{\partial \phi_{N}}{\partial \xi}(\xi, \eta)=0, \quad \frac{\partial \phi_{N}}{\partial \eta}(\xi, \eta)=\frac{1}{N} \theta_{2}^{\prime}\left(\frac{\eta}{N}\right), \\
L_{-m} \phi_{N}(\xi, \eta)=\frac{1}{N^{2}} \theta_{2}^{\prime \prime}\left(\frac{\eta}{N}\right),
\end{gathered}
$$

which give us

$$
\sup _{D_{2}}\left|\frac{\partial \phi_{N}}{\partial \xi}\right|=0, \quad \sup _{D_{2}}\left|\frac{\partial \phi_{N}}{\partial \eta}\right|=\mathrm{O}\left(\frac{1}{N}\right), \quad \sup _{D_{2}}\left|L_{-m}\left(\phi_{N}\right)\right|=\mathrm{O}\left(\frac{1}{N^{2}}\right) .
$$

The same works for $D_{4}$.

For $(\xi, \eta) \in D_{3}, \phi_{N}(\xi, \eta)=\theta_{2}(\xi / N)$ and thus

$$
\begin{gathered}
\frac{\partial \phi_{N}}{\partial \xi}(\xi, \eta)=\frac{1}{N} \theta_{2}^{\prime}\left(\frac{\xi}{N}\right), \quad \frac{\partial \phi_{N}}{\partial \eta}(\xi, \eta)=0, \\
L_{-m} \phi_{N}(\xi, \eta)=\frac{1}{N^{2}} \theta_{2}^{\prime \prime}\left(\frac{\xi}{N}\right)-\frac{1}{N} \frac{m}{\xi} \theta_{2}^{\prime}\left(\frac{\xi}{N}\right),
\end{gathered}
$$

which give us

$$
\sup _{D_{3}}\left|\frac{\partial \phi_{N}}{\partial \xi}\right|=\mathrm{O}\left(\frac{1}{N}\right), \quad \sup _{D_{3}}\left|\frac{\partial \phi_{N}}{\partial \eta}\right|=0, \quad \sup _{D_{3}}\left|L_{-m}\left(\phi_{N}\right)\right|=\mathrm{O}\left(\frac{1}{N^{2}}\right) .
$$

For $(\xi, \eta) \in D_{5}, \phi_{N}(\xi, \eta)=\theta_{1}(N \xi) \theta_{2}(\eta / N)$ and thus 


$$
\begin{aligned}
\frac{\partial \phi_{N}}{\partial \xi}(\xi, \eta)=N \theta_{1}^{\prime}(N \xi) \theta_{2}\left(\frac{\eta}{N}\right), \quad \frac{\partial \phi_{N}}{\partial \eta}(\xi, \eta) & =\frac{1}{N} \theta_{1}(N \xi) \theta_{2}^{\prime}\left(\frac{\eta}{N}\right) \\
L_{-m} \phi_{N}(\xi, \eta)=N^{2} \theta_{1}^{\prime \prime}(N \xi) \theta_{2}\left(\frac{\eta}{N}\right) & +\frac{1}{N^{2}} \theta_{1}(N \xi) \theta_{2}^{\prime \prime}\left(\frac{\eta}{N}\right) \\
& -\frac{m}{\xi} N \theta_{1}^{\prime}(N \xi) \theta_{2}\left(\frac{\eta}{N}\right)
\end{aligned}
$$

which give us

$$
\sup _{D_{5}}\left|\frac{\partial \phi_{N}}{\partial \xi}\right|=\mathrm{O}(N), \quad \sup _{D_{5}}\left|\frac{\partial \phi_{N}}{\partial \eta}\right|=\mathrm{O}\left(\frac{1}{N}\right), \quad \sup _{D_{5}}\left|L_{-m}\left(\phi_{N}\right)\right|=\mathrm{O}\left(N^{2}\right)
$$

The same works for $D_{8}$.

For $(\xi, \eta) \in D_{6}, \phi_{N}(\xi, \eta)=\theta_{2}(\xi / N) \theta_{2}(\eta / N)$ and thus

$$
\begin{array}{r}
\frac{\partial \phi_{N}}{\partial \xi}(\xi, \eta)=\frac{1}{N} \theta_{2}^{\prime}\left(\frac{\xi}{N}\right) \theta_{2}\left(\frac{\eta}{N}\right), \quad \frac{\partial \phi_{N}}{\partial \eta}(\xi, \eta)=\frac{1}{N} \theta_{2}\left(\frac{\xi}{N}\right) \theta_{2}^{\prime}\left(\frac{\eta}{N}\right) \\
L_{-m} \phi_{N}(\xi, \eta)=\frac{1}{N^{2}} \theta_{2}^{\prime \prime}\left(\frac{\xi}{N}\right) \theta_{2}\left(\frac{\eta}{N}\right)+\frac{1}{N^{2}} \theta_{2}\left(\frac{\xi}{N}\right) \theta_{2}^{\prime \prime}\left(\frac{\eta}{N}\right) \\
-\frac{m}{N \xi} \theta_{2}^{\prime}\left(\frac{\xi}{N}\right) \theta_{2}\left(\frac{\eta}{N}\right),
\end{array}
$$

which give us

$$
\sup _{D_{6}}\left|\frac{\partial \phi_{N}}{\partial \xi}\right|=\mathrm{O}\left(\frac{1}{N}\right), \quad \sup _{D_{6}}\left|\frac{\partial \phi_{N}}{\partial \eta}\right|=\mathrm{O}\left(\frac{1}{N}\right), \quad \sup _{D_{6}}\left|L_{-m}\left(\phi_{N}\right)\right|=\mathrm{O}\left(\frac{1}{N^{2}}\right) .
$$

The same works for $D_{7}$.

We now estimate the following quantities for $i \in\{1, \ldots, 8\}$ :

$$
\int_{D_{i}}\left|F_{m}\right| d \xi d \eta, \quad \int_{D_{i}}\left|\partial_{\xi} F_{m}\right| d \xi d \eta \quad \int_{D_{i}}\left|\partial_{\eta} F_{m}\right| d \xi d \eta
$$

Lemma 5.5 For $\operatorname{Re} m<1$, we have:

- for $i=1$,

$$
\int_{D_{i}}\left|F_{m}\right| d \xi d \eta=\mathrm{O}\left(\frac{1}{N^{2}}\right), \quad \int_{D_{i}}\left|\frac{\partial F_{m}}{\partial \xi}\right| d \xi d \eta=\mathrm{O}\left(\frac{1}{N}\right)
$$

- for $i=2,4$,

$$
\int_{D_{i}}\left|F_{m}\right| d \xi d \eta=\mathrm{O}\left(N^{2}\right), \quad \int_{D_{i}}\left|\frac{\partial F_{m}}{\partial \eta}\right| d \xi d \eta=\mathrm{O}(N)
$$


- for $i=3$,

$$
\int_{D_{i}}\left|F_{m}\right| d \xi d \eta=\mathrm{O}\left(N^{2}\right), \quad \int_{D_{i}}\left|\frac{\partial F_{m}}{\partial \xi}\right| d \xi d \eta=\mathrm{O}(N)
$$

- for $i=5,8$,

$$
\begin{gathered}
\int_{D_{i}}\left|F_{m}\right| d \xi d \eta=\mathrm{O}\left(\frac{1}{N^{2}}\right), \quad \int_{D_{i}}\left|\frac{\partial F_{m}}{\partial \xi}\right| d \xi d \eta=\mathrm{O}\left(\frac{1}{N}\right), \\
\int_{D_{i}}\left|\frac{\partial F_{m}}{\partial \eta}\right| d \xi d \eta=\mathrm{O}\left(\frac{1}{N^{2}}\right)
\end{gathered}
$$

- for $i=6,7$,

$$
\begin{gathered}
\int_{D_{i}}\left|F_{m}\right| d \xi d \eta=\mathrm{O}\left(N^{2}\right), \quad \int_{D_{i}}\left|\frac{\partial F_{m}}{\partial \xi}\right| d \xi d \eta=\mathrm{O}(N), \\
\int_{D_{i}}\left|\frac{\partial F_{m}}{\partial \eta}\right| d \xi d \eta=\mathrm{O}(N) .
\end{gathered}
$$

Proof By definition, for $\operatorname{Re} m<1$,

$$
F_{m}(\xi, \eta)=-\frac{\xi x^{1-m}}{2 \pi} \int_{0}^{\pi} \frac{\sin ^{1-m} \theta d \theta}{\left[(x-\xi)^{2}+4 x \xi \sin ^{2} \theta / 2+(y-\eta)^{2}\right]^{1-m / 2}} .
$$

Therefore there is a constant $C_{1}$ such that for all $(\xi, \eta) \in \mathbb{H}^{+}$, we have

$$
\left|F_{m}(\xi, \eta)\right| \leq \frac{C_{1} \xi}{\left[(x-\xi)^{2}+(\eta-y)^{2}\right]^{1-\operatorname{Re} m / 2}} .
$$

Similarly, we have

$$
\frac{\partial F_{m}}{\partial \xi}=\frac{F_{m}}{\xi}-\frac{\xi x^{1-m}}{2 \pi}(m-2) \int_{0}^{\pi} \frac{\left[(\xi-x)+2 x \sin ^{2} \theta / 2\right] \sin ^{1-m} \theta d \theta}{\left[(x-\xi)^{2}+4 x \xi \sin ^{2} \theta / 2+(y-\eta)^{2}\right]^{2-m / 2}},
$$

and as before, as for all $\theta \in[0, \pi]$,

$$
\begin{gathered}
\left|\frac{\left[(\xi-x)+2 x \sin ^{2} \theta / 2\right] \sin ^{1-m} \theta}{\left[(x-\xi)^{2}+4 x \xi \sin ^{2} \theta / 2+(y-\eta)^{2}\right]^{2-m / 2}}\right| \leq \frac{\left|(\xi-x)+2 x \sin ^{2} \theta / 2\right|}{\left[(x-\xi)^{2}+(\eta-y)^{2}\right]^{2-\operatorname{Re} m / 2}} \\
=\frac{|\xi-x \cos \theta|}{\left[(x-\xi)^{2}+(\eta-y)^{2}\right]^{2-\operatorname{Re} m / 2}} \leq \frac{\xi+x}{\left[(x-\xi)^{2}+(\eta-y)^{2}\right]^{2-\operatorname{Re} m / 2}},
\end{gathered}
$$


there exists a constant $C_{2}$ such that for all $N$ large enough and all $(\xi, \eta) \in \mathbb{H}^{+}$, we have

$$
\begin{aligned}
\left|\frac{\partial F_{m}}{\partial \xi}\right| \leq C_{2}\left[\frac{1}{\left[(x-\xi)^{2}+(\eta-y)^{2}\right]^{1-\operatorname{Re} m / 2}}\right. \\
\left.+\frac{\xi(x+\xi)}{\left[(x-\xi)^{2}+(\eta-y)^{2}\right]^{2-\operatorname{Re} m / 2}}\right] .
\end{aligned}
$$

Finally, as

$$
\frac{\partial F_{m}}{\partial \eta}=(2-m)(\eta-y) \frac{\xi x^{1-m}}{2 \pi} \int_{0}^{\pi} \frac{\sin ^{1-m} \theta d \theta}{\left[(x-\xi)^{2}+4 x \xi \sin ^{2} \theta / 2+(y-\eta)^{2}\right]^{2-m / 2}}
$$

there exists a constant $C_{3}$ such that for all $N$ large enough and all $(\xi, \eta) \in \mathbb{H}^{+}$, we have

$$
\left|\frac{\partial F_{m}}{\partial \eta}\right| \leq \frac{C_{3} \xi}{|\eta-y|^{3-\operatorname{Re} m}}
$$

Using these inequalities, we estimate integrals on domains $D_{i}$.

On $D_{1}$ : Inequality (5) implies

$$
\begin{aligned}
\int_{D_{1}}\left|F_{m}\right| d \xi d \eta & =\mathrm{O}(1) \int_{1 / 2 N}^{1 / N} d \xi \int_{-N / 2}^{N / 2} \frac{\xi d \eta}{\left[(x-\xi)^{2}+(\eta-y)^{2}\right]^{1-\operatorname{Re} m / 2}} \\
& =\mathrm{O}\left(\frac{1}{N^{2}}\right) \int_{-N / 2}^{N / 2} \frac{d \eta}{\left[(x-1 / N)^{2}+(\eta-y)^{2}\right]^{1-\operatorname{Re} m / 2}}=\mathrm{O}\left(\frac{1}{N^{2}}\right)
\end{aligned}
$$

Then, thanks to (6), we have

$$
\begin{aligned}
\int_{D_{1}}\left|\frac{\partial F_{m}}{\partial \xi}\right| d \xi d \eta & =\mathrm{O}(1) \int_{1 / 2 N}^{1 / N} d \xi \int_{-N / 2}^{N / 2}\left[\frac{1}{\left[(x-\xi)^{2}+(\eta-y)^{2}\right]^{1-\operatorname{Re} m / 2}}\right. \\
& \left.+\frac{\xi(x+\xi)}{\left[(x-\xi)^{2}+(\eta-y)^{2}\right]^{2-\operatorname{Re} m / 2}}\right] d \eta \\
= & \mathrm{O}\left(\frac{1}{N}\right) \int_{-N / 2}^{N / 2} \frac{d \eta}{\left[(x-1 / N)^{2}+(\eta-y)^{2}\right]^{1-\operatorname{Re} m / 2}}+\mathrm{O}\left(\frac{1}{N^{2}}\right) \\
= & \mathrm{O}\left(\frac{1}{N}\right) .
\end{aligned}
$$


On $D_{2}$ : Due to inequality (5), we have

$$
\begin{aligned}
\int_{D_{2}}\left|F_{m}\right| d \xi d \eta & =\mathrm{O}(1) \int_{1 / N}^{N / 2} d \xi \int_{N / 2}^{N} \frac{\xi d \eta}{\left[(x-\xi)^{2}+(\eta-y)^{2}\right]^{1-\operatorname{Re} m / 2}} \\
& =\mathrm{O}(1) \int_{1 / N}^{N / 2} d \xi \int_{N / 2}^{N} \frac{\xi d \eta}{|\eta-y|^{2-\operatorname{Re} m}} \\
& =\mathrm{O}\left(N^{2}\right) \int_{N / 2}^{N} \frac{d \eta}{|\eta-y|^{2-\operatorname{Re} m}} \\
& =\mathrm{O}\left(N^{2}\right)\left[\frac{1}{(N-y)^{1-\operatorname{Re} m}}-\frac{1}{(N / 2-y)^{1-\operatorname{Re} m}}\right]=\mathrm{O}\left(N^{\operatorname{Re} m+1}\right)
\end{aligned}
$$

Then, thanks to (7), we have

$$
\begin{aligned}
\int_{D_{2}}\left|\frac{\partial F_{m}}{\partial \eta}\right| d \xi d \eta & =\mathrm{O}(1) \int_{1 / N}^{N / 2} d \xi \int_{N / 2}^{N} \frac{\xi d \eta}{|\eta-y|^{3-\operatorname{Re} m}} \\
& =\mathrm{O}\left(N^{2}\right) \int_{N / 2}^{N} \frac{d \eta}{|\eta-y|^{3-\operatorname{Re} m}}=\mathrm{O}\left(N^{\operatorname{Re} m}\right)
\end{aligned}
$$

On $D_{3}$ : Due to inequality (5), we have

$$
\begin{aligned}
\int_{D_{3}}\left|F_{m}\right| d \xi d \eta & =\mathrm{O}(1) \int_{N / 2}^{N} d \xi \int_{-N / 2}^{N / 2} \frac{\xi d \eta}{\left[(x-\xi)^{2}+(\eta-y)^{2}\right]^{1-\operatorname{Re} m / 2}} \\
& =\mathrm{O}(1) \int_{N / 2}^{N} d \xi \int_{-N / 2}^{N / 2} \frac{\xi d \eta}{\left[(x-N / 2)^{2}+(\eta-y)^{2}\right]^{1-\operatorname{Re} m / 2}} \\
& =\mathrm{O}\left(N^{2}\right) \int_{-N / 2}^{N / 2} \frac{d \eta}{\left[(x-N / 2)^{2}+(\eta-y)^{2}\right]^{1-\operatorname{Re} m / 2}} \\
& =\mathrm{O}\left(N^{2}\right) \int_{-N / 2}^{N / 2} \frac{d \eta}{\left[1+(\eta-y)^{2}\right]^{1-\operatorname{Re} m / 2}}=\mathrm{O}\left(N^{2}\right) .
\end{aligned}
$$

Then, thanks to (6), we have

$$
\begin{aligned}
\int_{D_{3}}\left|\frac{\partial F_{m}}{\partial \xi}\right| d \xi d \eta & =\mathrm{O}(1) \int_{N / 2}^{N} d \xi \int_{-N / 2}^{N / 2}\left[\frac{1}{\left[(x-\xi)^{2}+(\eta-y)^{2}\right]^{1-\operatorname{Re} m / 2}}\right. \\
& \left.+\frac{\xi(x+\xi)}{\left[(x-\xi)^{2}+(\eta-y)^{2}\right]^{2-\operatorname{Re} m / 2}}\right] d \eta \\
& =\mathrm{O}(N) \int_{-N / 2}^{N / 2} \frac{d \eta}{\left[(x-N / 2)^{2}+(\eta-y)^{2}\right]^{1-\operatorname{Re} m / 2}}
\end{aligned}
$$




$$
\begin{aligned}
& +\mathrm{O}\left(N^{3}\right) \int_{-N / 2}^{N / 2} \frac{d \eta}{\left[(x-N / 2)^{2}+(\eta-y)^{2}\right]^{2-\operatorname{Re} m / 2}} \\
= & \mathrm{O}(N)+\mathrm{O}\left(N^{3}\right) \int_{-N / 2}^{N / 2} \frac{d \eta}{(x-N / 2)^{4-\operatorname{Re} m}} \\
= & \mathrm{O}(N)+\mathrm{O}\left(N^{\operatorname{Re} m}\right)=\mathrm{O}(N) .
\end{aligned}
$$

On $D_{4}$ : This case is analogous to the case $D_{2}$.

On $D_{5}$ : Due to inequality (5), we have

$$
\begin{aligned}
\int_{D_{5}}\left|F_{m}\right| d \xi d \eta & =\mathrm{O}(1) \int_{1 / 2 N}^{1 / N} d \xi \int_{N / 2}^{N} \frac{\xi d \eta}{\left[(x-\xi)^{2}+(\eta-y)^{2}\right]^{1-\operatorname{Re} m / 2}} \\
& =\mathrm{O}\left(\frac{1}{N^{2}}\right) \int_{N / 2}^{N} \frac{d \eta}{(\eta-y)^{2-\operatorname{Re} m}} \\
& =\mathrm{O}\left(\frac{1}{N^{2}}\right)\left[\frac{1}{(N-y)^{1-\operatorname{Re} m}}-\frac{1}{(N / 2-y)^{1-\operatorname{Re} m}}\right]=\mathrm{O}\left(\frac{1}{N^{3-\operatorname{Re} m}}\right) .
\end{aligned}
$$

Then, thanks to (6), we have

$$
\begin{aligned}
& \int_{D_{5}}\left|\frac{\partial F_{m}}{\partial \xi}\right| d \xi d \eta=\mathrm{O}(1) \int_{1 / 2 N}^{1 / N} d \xi \int_{N / 2}^{N}\left[\frac{1}{\left[(x-\xi)^{2}+(\eta-y)^{2}\right]^{1-\operatorname{Re} m / 2}}\right.\left.+\frac{\xi(x+\xi)}{\left.\left[(x-\xi)^{2}+(\eta-y)^{2}\right]^{2-\operatorname{Re} m / 2}\right]}\right] d \eta \\
&=\mathrm{O}(1) \int_{1 / 2 N}^{1 / N} d \xi \int_{N / 2}^{N}\left[\frac{1}{\left[(x-1 / N)^{2}+(\eta-y)^{2}\right]^{1-\operatorname{Re} m / 2}}\right. \\
&\left.+\frac{\xi(x+\xi)}{\left.\left[(x-1 / N)^{2}+(\eta-y)^{2}\right]^{2-\operatorname{Re} m / 2}\right]}\right] \\
&=\mathrm{O}\left(\frac{1}{N}\right) .
\end{aligned}
$$

Estimate (7) gives

$$
\int_{D_{5}}\left|\frac{\partial F_{m}}{\partial \eta}\right| d \xi d \eta=\mathrm{O}(1) \int_{1 / 2 N}^{1 / N} d \xi \int_{N / 2}^{N} \frac{\xi d \eta}{|\eta-y|^{3-\operatorname{Re} m}}=\mathrm{O}\left(\frac{1}{N^{2}}\right)
$$


On $D_{6}$ : Due to (5), we have

$$
\begin{aligned}
\int_{D_{6}}\left|F_{m}\right| d \xi d \eta & =\mathrm{O}(1) \int_{N / 2}^{N} d \xi \int_{N / 2}^{N} \frac{\xi d \eta}{\left[(x-\xi)^{2}+(\eta-y)^{2}\right]^{1-\operatorname{Re} m / 2}} \\
& =\mathrm{O}\left(N^{2}\right) \int_{N / 2}^{N} \frac{d \eta}{(\eta-y)^{2-\operatorname{Re} m}} \\
& =\mathrm{O}\left(N^{2}\right)\left[\frac{1}{(N-y)^{1-\operatorname{Re} m}}-\frac{1}{(N / 2-y)^{1-\operatorname{Re} m}}\right]=\mathrm{O}\left(N^{1+\operatorname{Re} m}\right) .
\end{aligned}
$$

Then, thanks to (6), we have

$$
\begin{aligned}
\int_{D_{6}}\left|\frac{\partial F_{m}}{\partial \xi}\right| d \xi d \eta & =\mathrm{O}(1) \int_{N / 2}^{N} d \xi \int_{N / 2}^{N}\left[\frac{1}{\left[(x-\xi)^{2}+(\eta-y)^{2}\right]^{1-\operatorname{Re} m / 2}}\right. \\
& \left.+\frac{\xi(x+\xi)}{\left[(x-\xi)^{2}+(\eta-y)^{2}\right]^{2-\operatorname{Re} m / 2}}\right] d \eta \\
& =\mathrm{O}(1) \int_{N / 2}^{N} d \xi \int_{N / 2}^{N}\left[\frac{1}{(\eta-y)^{2-\operatorname{Re} m}}+\frac{\xi(x+\xi)}{(\eta-y)^{4-\operatorname{Re} m}}\right] d \eta \\
& =\mathrm{O}(N)+\mathrm{O}\left(N^{3}\right) \int_{N / 2}^{N} \frac{d \eta}{(\eta-y)^{4-\operatorname{Re} m}}=\mathrm{O}(N)+\mathrm{O}\left(N^{\operatorname{Re} m}\right) \\
& =\mathrm{O}(N) .
\end{aligned}
$$

Estimate (7) gives

$$
\begin{aligned}
\int_{D_{6}}\left|\frac{\partial F_{m}}{\partial \eta}\right| d \xi d \eta & =\mathrm{O}(1) \int_{N / 2}^{N} d \xi \int_{N / 2}^{N} \frac{\xi d \eta}{|\eta-y|^{3-\operatorname{Re} m}} \\
& =O\left(N^{2}\right) \int_{N / 2}^{N} \frac{d \eta}{|\eta-y|^{3-\operatorname{Re} m}}=\mathrm{O}\left(N^{\operatorname{Re} m}\right)
\end{aligned}
$$

On $D_{7}, D_{8}$ : These cases are analogous to the cases $D_{6}$ and $D_{5}$, respectively.

Lemma 5.6 Lemma 5.5 remains true for $\operatorname{Re} m \geq 1$.

Proof For $\operatorname{Re} m \geq 1$, we have

$$
\begin{array}{r}
F_{m}(x, y, \xi, \eta)=-\frac{\xi^{m}}{2 \pi} \int_{0}^{\pi} \sin ^{m-1} \theta\left(\frac{1}{\left[(x-\xi)^{2}+4 x \xi \sin ^{2} \theta / 2+(y-\eta)^{2}\right]^{m / 2}}\right. \\
\left.-\frac{1}{\left[(x+\xi)^{2}-4 x \xi \sin ^{2} \theta / 2+(y-\eta)^{2}\right]^{m / 2}}\right) d \theta .
\end{array}
$$


Since for all $(\xi, \eta) \in \mathbb{H}^{+}$, we have

$$
\left|\left[(x+\xi)^{2}-4 x \xi \sin ^{2} \frac{\theta}{2}+(y-\eta)^{2}\right]^{m / 2}\right|=\left|\left[x^{2}+\xi^{2}+2 x \xi \cos \theta+(y-\eta)^{2}\right]^{m / 2}\right|,
$$

then

$$
\left|\left[(x+\xi)^{2}-4 x \xi \sin ^{2} \frac{\theta}{2}+(y-\eta)^{2}\right]^{m / 2}\right| \geq\left[(x-\xi)^{2}+(y-\eta)^{2}\right]^{\operatorname{Re} m / 2}
$$

and there is a constant $C_{1}^{\prime}$ such that for all $(\xi, \eta) \in \mathbb{H}^{+}$, we have

$$
\left|F_{m}\right| \leq \frac{C_{1}^{\prime} \xi^{\operatorname{Re} m}}{\left[(x-\xi)^{2}+(y-\eta)^{2}\right]^{\operatorname{Re} m / 2}} .
$$

This inequality does not suffice to estimate integrals over $D_{1}$. We shall improve it as follows. Rewrite $F_{m}$ as

$$
F_{m}(x, y, \xi, \eta)=-\frac{\xi^{m}}{2 \pi} \int_{0}^{\pi} \sin ^{m-1} \theta K_{m}(x, y, \xi, \eta, \theta) d \theta
$$

where

$$
\begin{aligned}
K_{m}(x, y, \xi, \eta, \theta)= & \frac{1}{\left[(x-\xi)^{2}+4 x \xi \sin ^{2} \theta / 2+(y-\eta)^{2}\right]^{m / 2}} \\
& \quad-\frac{1}{\left[(x+\xi)^{2}-4 x \xi \sin ^{2} \theta / 2+(y-\eta)^{2}\right]^{m / 2}}
\end{aligned}
$$

For $(x, y) \in \mathbb{H}^{+}, \theta \in[0, \pi]$ and $\eta \in \mathbb{R}$ fixed, define a function $g_{m}$ on $[-1 / N, 1 / N]$, with $1 / N<x$, by

$$
g_{m}(\xi)=\frac{1}{\left[(x-\xi)^{2}+4 x \xi \sin ^{2} \theta / 2+(y-\eta)^{2}\right]^{m / 2}} .
$$

This function is well defined because

$$
\begin{aligned}
(x-\xi)^{2}+4 x \xi \sin ^{2} \frac{\theta}{2}+(y-\eta)^{2} & =x^{2}+\xi^{2}-2 x \xi \cos \theta+(y-\eta)^{2} \\
& \geq(x-|\xi|)^{2}+(y-\eta)^{2}
\end{aligned}
$$

and the last term is greater than $(x-1 / N)^{2}>0$.

We have

$$
K_{m}(x, y, \xi, \eta, \theta)=g_{m}(\xi)-g_{m}(-\xi)
$$


thus

$$
\left|K_{m}(x, y, \xi, \eta, \theta)\right| \leq 2 \xi \sup _{[-\xi, \xi]}\left|g_{m}^{\prime}\right| \leq 2|m| \xi \frac{|\xi-x|+2 x}{\left[(x-\xi)^{2}+(y-\eta)^{2}\right]^{1+\operatorname{Re} m / 2}},
$$

which implies that there exists a constant $c_{1}^{\prime}$ such that for all $(\xi, \eta) \in D_{1}$,

$$
\left|F_{m}\right| \leq c_{1}^{\prime} \frac{\xi^{\operatorname{Re} m+1}}{\left[(x-\xi)^{2}+(\eta-y)^{2}\right]^{1+\operatorname{Re} m / 2}} .
$$

Similarly, we have

$$
\begin{aligned}
\frac{\partial F_{m}}{\partial \xi}= & \frac{m F_{m}}{\xi} \\
+ & \frac{m \xi^{m}}{2 \pi} \int_{0}^{\pi} \sin ^{m-1} \theta\left(\frac{(\xi-x)+2 x \sin ^{2} \theta / 2}{\left[(x-\xi)^{2}+4 x \xi \sin ^{2} \theta / 2+(y-\eta)^{2}\right]^{m / 2+1}}\right. \\
& \left.\quad-\frac{(\xi+x)-2 x \sin ^{2} \theta / 2}{\left[(x+\xi)^{2}-4 x \xi \sin ^{2} \theta / 2+(y-\eta)^{2}\right]^{m / 2+1}}\right) d \theta .
\end{aligned}
$$

and as before, for all $\theta \in[0, \pi]$,

$$
\begin{aligned}
& \left|\frac{\left[(\xi-x)+2 x \sin ^{2} \theta / 2\right] \sin ^{m-1} \theta}{\left[(x-\xi)^{2}+4 x \xi \sin ^{2} \theta / 2+(y-\eta)^{2}\right]^{m / 2+1}}\right| \leq \frac{\left|(\xi-x)+2 x \sin ^{2} \theta / 2\right|}{\left[(x-\xi)^{2}+(y-\eta)^{2}\right]^{\operatorname{Re} m / 2+1}} \\
& \quad=\frac{|\xi-x \cos \theta|}{\left[(x-\xi)^{2}+(y-\eta)^{2}\right]^{\operatorname{Re} m / 2+1}} \leq \frac{\xi+x}{\left[(x-\xi)^{2}+(y-\eta)^{2}\right]^{\operatorname{Re} m / 2+1}}
\end{aligned}
$$

and thanks to (8), for all $\theta \in[0, \pi]$,

$$
\begin{aligned}
& \left|\frac{\left[(\xi+x)-2 x \sin ^{2} \theta / 2\right] \sin ^{m-1} \theta}{\left[(x+\xi)^{2}-4 x \xi \sin ^{2} \theta / 2+(y-\eta)^{2}\right]^{m / 2+1}}\right| \leq \frac{\left|(\xi+x)-2 x \sin ^{2} \theta / 2\right|}{\left[(x-\xi)^{2}+(y-\eta)^{2}\right]^{\operatorname{Re} m / 2+1}} \\
& \quad=\frac{|\xi+x \cos \theta|}{\left[(x-\xi)^{2}+(y-\eta)^{2}\right]^{\operatorname{Re} m / 2+1}} \leq \frac{\xi+x}{\left[(x-\xi)^{2}+(y-\eta)^{2}\right]^{\operatorname{Re} m / 2+1}} .
\end{aligned}
$$

These estimates, (11) and (9) show that there is a constant $C_{2}^{\prime}$ such that for large enough $N$ and all $(\xi, \eta) \in \mathbb{H}^{+}$, we have

$$
\begin{aligned}
\left|\frac{\partial F_{m}}{\partial \xi}\right| \leq C_{2}^{\prime}\left(\frac{\xi^{\operatorname{Re} m-1}}{\left[(x-\xi)^{2}+(y-\eta)^{2}\right]^{\operatorname{Re} m / 2}}\right. & \\
& \left.+\frac{\xi^{\operatorname{Re} m}(\xi+x)}{\left[(x-\xi)^{2}+(y-\eta)^{2}\right]^{\operatorname{Re} m / 2+1}}\right) .
\end{aligned}
$$


We can improve this inequality on $D_{1}$, by using inequality (10) instead of (9), then there are two constants $C_{2}^{\prime \prime}$ and $C_{2}^{\prime \prime \prime}$ (which do not depend on $N$ ) such that for all $(\xi, \eta) \in D_{1}$,

$$
\begin{aligned}
&\left|\frac{\partial F_{m}}{\partial \xi}\right| \leq C_{2}^{\prime \prime}\left(\frac{\xi^{\operatorname{Re} m}}{\left[(x-\xi)^{2}+(y-\eta)^{2}\right]^{1+\operatorname{Re} m / 2}}\right. \\
&\left.+\frac{\xi^{\operatorname{Re} m}(\xi+x)}{\left[(x-\xi)^{2}+(y-\eta)^{2}\right]^{\operatorname{Re} m / 2+1}}\right) \\
& \leq C_{2}^{\prime \prime \prime} \frac{\xi^{\operatorname{Re} m}}{\left[(x-\xi)^{2}+(y-\eta)^{2}\right]^{1+\operatorname{Re} m / 2}} .
\end{aligned}
$$

Finally,

$$
\begin{array}{r}
\frac{\partial F_{m}}{\partial \eta}=\frac{m(\eta-y) \xi^{m}}{2 \pi} \int_{0}^{\pi} \sin ^{m-1} \theta\left(\frac{1}{\left[(x-\xi)^{2}+4 x \xi \sin ^{2} \theta / 2+(y-\eta)^{2}\right]^{m / 2+1}}\right. \\
\left.-\frac{1}{\left[(x+\xi)^{2}-4 x \xi \sin ^{2} \theta / 2+(y-\eta)^{2}\right]^{m / 2+1}}\right) d \theta
\end{array}
$$

Similarly, there is a constant $C_{3}^{\prime}$ such that for all $N$ large enough and all $(\xi, \eta) \in \mathbb{H}^{+}$, we have

$$
\left|\frac{\partial F_{m}}{\partial \eta}\right| \leq C_{3}^{\prime} \frac{|\eta-y| \xi^{\operatorname{Re} m}}{\left[(x-\xi)^{2}+(y-\eta)^{2}\right]^{\operatorname{Re} m / 2+1}} .
$$

Thanks to these inequalities, we can now estimate the corresponding integrals over domains $D_{i}$.

On $D_{1}$ : Due to (10), we have

$$
\begin{aligned}
\int_{D_{1}}\left|F_{m}\right| d \xi d \eta & =\mathrm{O}(1) \int_{1 / 2 N}^{1 / N} d \xi \int_{-N / 2}^{N / 2} \frac{\xi^{\operatorname{Re} m+1} d \eta}{\left[(x-\xi)^{2}+(\eta-y)^{2}\right]^{1+\operatorname{Re} m / 2}} \\
& =\mathrm{O}(N) \int_{1 / 2 N}^{1 / N} \xi^{\operatorname{Re} m+1} d \xi=\mathrm{O}(N)\left[\left(\frac{1}{N}\right)^{\operatorname{Re} m+2}-\left(\frac{1}{2 N}\right)^{\operatorname{Re} m+2}\right] \\
& =\mathrm{O}\left(\frac{1}{N^{\operatorname{Re} m+1}}\right) .
\end{aligned}
$$

Then thanks to (13),

$$
\begin{aligned}
\int_{D_{1}}\left|\frac{\partial F_{m}}{\partial \xi}\right| d \xi d \eta & =\mathrm{O}(1) \int_{1 / 2 N}^{1 / N} d \xi \int_{-N / 2}^{N / 2} \frac{\xi^{\operatorname{Re} m} d \eta}{\left[(x-\xi)^{2}+(\eta-y)^{2}\right]^{1+\operatorname{Re} m / 2}} \\
& =\mathrm{O}(N) \int_{1 / 2 N}^{1 / N} \xi^{\operatorname{Re} m} d \xi=\mathrm{O}\left(\frac{1}{N^{\operatorname{Re} m}}\right) .
\end{aligned}
$$


On $D_{2}$ : Due to (9), we have

$$
\begin{aligned}
\int_{D_{2}}\left|F_{m}\right| d \xi d \eta & =\mathrm{O}(1) \int_{1 / N}^{N / 2} d \xi \int_{N / 2}^{N} \frac{\xi^{\operatorname{Re} m} d \eta}{\left[(x-\xi)^{2}+(y-\eta)^{2}\right]^{\operatorname{Re} m / 2}} \\
& =\mathrm{O}(1) \int_{1 / N}^{N / 2} d \xi \int_{N / 2}^{N} \frac{\xi^{\operatorname{Re} m} d \eta}{|y-N / 2|^{\operatorname{Re} m}}=\mathrm{O}\left(N^{2}\right)
\end{aligned}
$$

because we integrate a bounded function (independent of $N$ ) on a domain with measure controlled by $\mathrm{O}\left(N^{2}\right)$.

Then, inequality (14) implies

$$
\begin{aligned}
\int_{D_{2}}\left|\frac{\partial F_{m}}{\partial \eta}\right| d \xi d \eta & =\mathrm{O}(1) \int_{1 / N}^{N / 2} d \xi \int_{N / 2}^{N} \frac{|\eta-y| \xi^{\operatorname{Re} m} d \eta}{\left[(x-\xi)^{2}+(y-\eta)^{2}\right]^{\operatorname{Re} m / 2+1}} \\
& =\mathrm{O}(1) \int_{1 / N}^{N / 2} d \xi \int_{N / 2}^{N} \frac{\xi^{\operatorname{Re} m} d \eta}{|y-\eta|^{\operatorname{Re} m+1}} \\
& =\mathrm{O}(1) \int_{1 / N}^{N / 2} d \xi \int_{N / 2}^{N} \frac{N^{\operatorname{Re} m} d \eta}{|N / 2-y|^{\operatorname{Re} m+1}}=\mathrm{O}(N)
\end{aligned}
$$

On $D_{3}$ : Due to (9), we have

$$
\begin{aligned}
\int_{D_{3}}\left|F_{m}\right| d \xi d \eta & =\mathrm{O}(1) \int_{N / 2}^{N} d \xi \int_{-N / 2}^{N / 2} \frac{\xi^{\operatorname{Re} m} d \eta}{\left[(x-\xi)^{2}+(y-\eta)^{2}\right]^{\operatorname{Re} m / 2}} \\
& =\mathrm{O}(1) \int_{N / 2}^{N} d \xi \int_{-N / 2}^{N / 2} \frac{\xi^{\operatorname{Re} m} d \eta}{\left[(x-N / 2)^{2}+(y-\eta)^{2}\right]^{\operatorname{Re} m / 2}} \\
& =\mathrm{O}\left(N^{\operatorname{Re} m+1}\right) \int_{-N / 2}^{N / 2} \frac{d \eta}{\left[(x-N / 2)^{2}+(y-\eta)^{2}\right]^{\operatorname{Re} m / 2}}=\mathrm{O}\left(N^{2}\right) .
\end{aligned}
$$

Then, thanks to (12), we have

$$
\begin{aligned}
\int_{D_{3}}\left|\frac{\partial F_{m}}{\partial \xi}\right| d \xi d \eta & =\mathrm{O}(1) \int_{N / 2}^{N} d \xi \int_{-N / 2}^{N / 2}\left(\frac{\xi^{\operatorname{Re} m-1}}{\left[(x-\xi)^{2}+(y-\eta)^{2}\right]^{\operatorname{Re} m / 2}}\right) d \eta \\
& \left.+\frac{\xi^{\operatorname{Re} m}(\xi+x)}{\left[(x-\xi)^{2}+(y-\eta)^{2}\right]^{\operatorname{Re} m / 2+1}}\right) d \eta \\
=\mathrm{O}\left(N^{\operatorname{Re} m}\right) \int_{-N / 2}^{N / 2} \frac{d \eta}{\left[(x-N / 2)^{2}+(y-\eta)^{2}\right]^{\operatorname{Re} m / 2}} & d \eta \\
+\mathrm{O}\left(N^{\operatorname{Re} m+2}\right) \int_{-N / 2}^{N / 2} \frac{\left.d(x-N / 2)^{2}+(y-\eta)^{2}\right]^{\operatorname{Re} m / 2+1}}{[(N)} & \mathrm{O}(N)+\mathrm{O}(N)=\mathrm{O}(N)
\end{aligned}
$$


On $D_{4}$ : This case is analogous to the case $D_{2}$.

On $D_{5}$ : Due to (9), we have

$$
\begin{aligned}
\int_{D_{5}}\left|F_{m}\right| d \xi d \eta & =\mathrm{O}(1) \int_{1 / 2 N}^{1 / N} d \xi \int_{N / 2}^{N} \frac{\xi^{\operatorname{Re} m} d \eta}{\left[(x-\xi)^{2}+(y-\eta)^{2}\right]^{\operatorname{Re} m / 2}} \\
& =\mathrm{O}\left(\frac{1}{N^{\operatorname{Re} m+1}}\right) \int_{N / 2}^{N} \frac{d \eta}{|y-N / 2|^{\operatorname{Re} m}}=\mathrm{O}\left(\frac{1}{N^{2 \operatorname{Re} m}}\right)
\end{aligned}
$$

Then, thanks to (12), we have

$$
\begin{aligned}
\int_{D_{5}}\left|\frac{\partial F_{m}}{\partial \xi}\right| d \xi d \eta & =\mathrm{O}(1) \int_{1 / 2 N}^{1 / N} d \xi \int_{N / 2}^{N}\left(\frac{\xi^{\operatorname{Re} m-1}}{\left[(x-\xi)^{2}+(y-\eta)^{2}\right]^{\operatorname{Re} m / 2}}\right. \\
& \left.+\frac{\xi^{\operatorname{Re} m}(\xi+x)}{\left[(x-\xi)^{2}+(y-\eta)^{2}\right]^{\operatorname{Re} m / 2+1}}\right) d \eta \\
& =\mathrm{O}(1) \int_{1 / 2 N}^{1 N} d \xi \int_{N / 2}^{N}\left(\frac{\xi^{\operatorname{Re} m-1}}{|y-\eta|^{\operatorname{Re} m}}+\frac{\xi^{\operatorname{Re} m}(\xi+x)}{|y-\eta|^{\operatorname{Re} m+2}}\right) d \eta \\
& =\mathrm{O}\left(\frac{1}{N^{2 \operatorname{Re} m-1}}\right) .
\end{aligned}
$$

Applying inequality (14), we have

$$
\begin{aligned}
\int_{D_{5}}\left|\frac{\partial F_{m}}{\partial \eta}\right| d \xi d \eta & =\mathrm{O}(1) \int_{1 / 2 N}^{1 / N} d \xi \int_{N / 2}^{N} \frac{|\eta-y| \xi^{\operatorname{Re} m} d \eta}{\left[(x-\xi)^{2}+(y-\eta)^{2}\right]^{\operatorname{Re} m / 2+1}} \\
& =\mathrm{O}(1) \int_{1 / 2 N}^{1 / N} d \xi \int_{N / 2}^{N} \frac{\xi^{\operatorname{Re} m} d \eta}{|y-\eta|^{\operatorname{Re} m+1}}=\mathrm{O}\left(\frac{1}{N^{2 \operatorname{Re} m+1}}\right)
\end{aligned}
$$

On $D_{6}$ : Due to (9), we have

$$
\begin{aligned}
\int_{D_{6}}\left|F_{m}\right| d \xi d \eta & =\mathrm{O}(1) \int_{N / 2}^{N} d \xi \int_{N / 2}^{N} \frac{\xi^{\operatorname{Re} m} d \eta}{\left[(x-\xi)^{2}+(y-\eta)^{2}\right]^{\operatorname{Re} m / 2}} \\
& =\mathrm{O}\left(N^{\operatorname{Re} m+1}\right) \int_{N / 2}^{N} \frac{d \eta}{(N / 2-y)^{\operatorname{Re} m}}=\mathrm{O}\left(N^{2}\right) .
\end{aligned}
$$


Then, thanks to (12), we obtain

$$
\begin{aligned}
\int_{D_{6}}\left|\frac{\partial F_{m}}{\partial \xi}\right| d \xi d \eta & =\mathrm{O}(1) \int_{N / 2}^{N} d \xi \int_{N / 2}^{N}\left(\frac{\xi^{\operatorname{Re} m-1}}{\left[(x-\xi)^{2}+(y-\eta)^{2}\right]^{\operatorname{Re} m / 2}}\right. \\
& \left.+\frac{\xi^{\operatorname{Re} m}(\xi+x)}{\left[(x-\xi)^{2}+(y-\eta)^{2}\right]^{\operatorname{Re} m / 2+1}}\right) d \eta \\
& =\mathrm{O}(1) \int_{N / 2}^{N} d \xi \int_{N / 2}^{N}\left(\frac{\xi^{\operatorname{Re} m-1}}{|y-\eta|^{\operatorname{Re} m}}+\frac{\xi^{\operatorname{Re} m}(\xi+x)}{|y-\eta|^{\operatorname{Re} m+2}}\right) d \eta \\
& =\mathrm{O}(N)+\mathrm{O}\left(N^{\operatorname{Re} m+2}\right) \int_{N / 2}^{N} \frac{d \eta}{|y-\eta|^{\operatorname{Re} m+2}}=\mathrm{O}(N) .
\end{aligned}
$$

Finally, inequality (14) implies

$$
\begin{aligned}
\int_{D_{6}}\left|\frac{\partial F_{m}}{\partial \eta}\right| d \xi d \eta & =\mathrm{O}(1) \int_{N / 2}^{N} d \xi \int_{N / 2}^{N} \frac{|\eta-y| \xi^{\operatorname{Re} m} d \eta}{\left[(x-\xi)^{2}+(y-\eta)^{2}\right]^{\operatorname{Re} m / 2+1}} \\
& =\mathrm{O}\left(N^{\operatorname{Re} m+1}\right) \int_{N / 2}^{N} \frac{d \eta}{|y-\eta|^{\operatorname{Re} m+1}}=\mathrm{O}(N) .
\end{aligned}
$$

On $D_{7}, D_{8}$ : These cases are analogous to the cases $D_{6}$ and $D_{8}$, respectively.

In the following table, we summarize results obtained on the previous lemmas:

\begin{tabular}{llllll}
\hline$i$ & $\sup _{D_{i}}\left|L_{-m} \phi_{N}\right|$ & $\int_{D_{i}}\left|F_{m}\right|$ & $\left(\left|\partial_{\xi} \phi_{N}\right|,\left|\partial_{\eta} \phi_{N}\right|\right)$ & $\int_{D_{i}}\left|\partial_{\xi} F_{m}\right|$ & $\int_{D_{i}}\left|\partial_{\eta} F_{m}\right|$ \\
\hline 1 & $\mathrm{O}\left(N^{2}\right)$ & $\mathrm{O}\left(1 / N^{2}\right)$ & $(\mathrm{O}(N), 0)$ & $\mathrm{O}(1 / N)$ & $\times$ \\
2 & $\mathrm{O}\left(1 / N^{2}\right)$ & $\mathrm{O}\left(N^{2}\right)$ & $(0, \mathrm{O}(1 / N))$ & $\times$ & $\mathrm{O}(N)$ \\
3 & $\mathrm{O}\left(1 / N^{2}\right)$ & $\mathrm{O}\left(N^{2}\right)$ & $(\mathrm{O}(1 / N), 0)$ & $\mathrm{O}(N)$ & $\times$ \\
4 & $\mathrm{O}\left(1 / N^{2}\right)$ & $\mathrm{O}\left(N^{2}\right)$ & $(0, \mathrm{O}(1 / N))$ & $\times$ & $\mathrm{O}(N)$ \\
5 & $\mathrm{O}\left(N^{2}\right)$ & $\mathrm{O}\left(1 / N^{2}\right)$ & $(\mathrm{O}(N), \mathrm{O}(1 / N))$ & $\mathrm{O}(1 / N)$ & $\mathrm{O}\left(1 / N^{2}\right)$ \\
6 & $\mathrm{O}\left(1 / N^{2}\right)$ & $\mathrm{O}\left(N^{2}\right)$ & $(\mathrm{O}(1 / N), \mathrm{O}(1 / N))$ & $\mathrm{O}(N)$ & $\mathrm{O}(N)$ \\
7 & $\mathrm{O}\left(1 / N^{2}\right)$ & $\mathrm{O}\left(N^{2}\right)$ & $(\mathrm{O}(1 / N), \mathrm{O}(1 / N))$ & $\mathrm{O}(N)$ & $\mathrm{O}(N)$ \\
8 & $\mathrm{O}\left(N^{2}\right)$ & $\mathrm{O}\left(1 / N^{2}\right)$ & $(\mathrm{O}(N), \mathrm{O}(1 / N))$ & $\mathrm{O}(1 / N)$ & $\mathrm{O}\left(1 / N^{2}\right)$ \\
\hline
\end{tabular}

We can easily check that for each $i \in\{1, \ldots, 8\}$, the quantities

$$
\sup _{D_{i}}\left|L_{-m} \phi_{N}\right| \int_{D_{i}}\left|F_{m}\right|, \quad \sup _{D_{i}}\left|\partial_{\xi} \phi_{N}\right| \int_{D_{i}}\left|\partial_{\xi} F_{m}\right|, \quad \sup _{D_{i}}\left|\partial_{\eta} \phi_{N}\right| \int_{D_{i}}\left|\partial_{\eta} F_{m}\right|
$$

are bounded. Therefore,

$$
u(x, y)=\mathrm{o}(1) \quad \text { as } \quad N \rightarrow+\infty .
$$

Thus $u \equiv 0$ and this completes the proof of Proposition 5.3. 
Proposition 5.7 Let $u \in \mathcal{D}\left(\mathbb{H}^{+}\right)$and let $(x, y) \in \mathbb{H}^{+}$, define

$$
U(x, y)=\int_{\mathbb{H}^{+}} u(\xi, \eta) F_{m}(x, y, \xi, \eta) d \xi d \eta
$$

then $\lim _{\|(x, y)\| \rightarrow+\infty} U=0$, and for all $y \in \mathbb{R}, \lim _{(0, y)} U=0$. Moreover, $U \in$ $C^{\infty}\left(\mathbb{H}^{+} \backslash \operatorname{supp} u\right)$ and for all $(x, y) \notin \operatorname{supp} u$ we have $L_{m, x, y} U(x, y)=0$.

Proof Fix $(\xi, \eta)$. For Re $m<1$,

$$
F_{m}(x, y, \xi, \eta)=-\frac{\xi x^{1-m}}{2 \pi} \int_{0}^{\pi} \frac{\sin ^{1-m} \theta d \theta}{\left[(x-\xi)^{2}+4 x \xi \sin ^{2} \theta / 2+(y-\eta)^{2}\right]^{1-m / 2}},
$$

hence $F_{m}(x, y, \xi, \eta) \rightarrow 0$ as $\|(x, y)\| \rightarrow+\infty$. For $\operatorname{Re} m \geq 1$,

$$
\begin{array}{r}
F_{m}(x, y, \xi, \eta)=-\frac{\xi^{m}}{2 \pi} \int_{0}^{\pi} \sin ^{m-1} \theta\left[\frac{1}{\left[(x-\xi)^{2}+4 x \xi \sin ^{2} \theta / 2+(y-\eta)^{2}\right]^{m / 2}}\right. \\
\left.-\frac{1}{\left[(x+\xi)^{2}-4 x \xi \sin ^{2} \theta / 2+(y-\eta)^{2}\right]^{m / 2}}\right] d \theta,
\end{array}
$$

hence $F_{m}(x, y, \xi, \eta) \rightarrow 0$ as $\|(x, y)\| \rightarrow+\infty$. So the first statement of the proposition is shown.

For the second statement, for $\operatorname{Re} m<1$, we have

$$
F_{m}(x, y, \xi, \eta) \sim-\frac{\xi x^{1-m}}{2 \pi\left[\xi^{2}+\left(y^{\prime}-\eta\right)^{2}\right]^{1-m / 2}} \int_{0}^{\pi} \sin ^{1-m} \theta d \theta
$$

as $(x, y) \rightarrow\left(0, y^{\prime}\right)$, which implies the desired result.

Now, assume that $\operatorname{Re} m \geq 1$. Let $(\xi, \eta)$ be fixed in the support of $u$, which is a compact set in $\mathbb{H}^{+}$. In particular, there exist $M>0$ and $\alpha>0$ which do not depend on $u$ such that $\|(\xi, \eta)\| \leq M$ and $\xi \geq 2 \alpha$. Let $y$ be in $\mathbb{R}$. Denote

$$
f_{m}(x)=\frac{1}{\left[(x-\xi)^{2}+4 x \xi \sin ^{2} \theta / 2+(y-\eta)^{2}\right]^{m / 2}}, \quad x \in[-\alpha, \alpha] .
$$

By the mean value inequality, for $x>0$ near 0 , we have

$$
\left|f_{m}(x)-f_{m}(0)\right| \leq x \sup _{[0, \alpha]}\left|f_{m}^{\prime}\right|
$$

and

$$
\left|f_{m}(-x)-f_{m}(0)\right| \leq x \sup _{[-\alpha, 0]}\left|f_{m}^{\prime}\right|,
$$


then

$$
\left|f_{m}(x)-f_{m}(-x)\right| \leq 2 x \sup _{[-\alpha, \alpha]}\left|f_{m}^{\prime}\right| \leq 2 x|m| \frac{3 M+\alpha}{\alpha^{\operatorname{Re} m+2}}
$$

In particular,

$$
\sup _{\substack{(\xi, \eta) \in \operatorname{supp} \\ y \in \mathbb{R}}}\left|F_{m}(x, y)\right|=\mathrm{O}(x)
$$

as $x \rightarrow 0+$. The second statement is proved.

The last statement can be deduced from the fact that if $(x, y) \neq(\xi, \eta)$ are both in $\mathbb{H}^{+}$, then

$$
L_{m, x, y} F_{m}(x, y, \xi, \eta)=0 .
$$

Remark 5.8 If $U \in \mathcal{D}\left(\mathbb{H}^{+}\right)$, then $L_{m, x, y} U=u$, but this identity is not necessarily true if $U \notin \mathcal{D}\left(\mathbb{H}^{+}\right)$. In particular, we cannot say that in Proposition 5.7 we have $L_{m} U=u$.

Now, we will prove a decomposition theorem for axisymmetric potentials, it is interesting to compare it with the known result in [6, Section 4, Theorem 2]. The fundamental difference is that in this paper, the conductivity is not extended by reflection through the boundary $\partial \Omega$ to the whole domain.

Note that, due to our construction of fundamental solutions, the proof of this theorem is more or less the same as the proof of the decomposition theorem in [4, Chapter 9]. Note also that in our situation, the domain of our functions is $\mathbb{H}^{+}$not $\mathbb{C}$.

Theorem 5.9 Let $m \in \mathbb{C}$. Let $\Omega$ be an open set in $\mathbb{H}^{+}$and let $K$ be a compact set in $\Omega$. If $u \in C^{2}(\Omega \backslash K)$ satisfies $L_{m} u=0$ in $\Omega \backslash K$, then $u$ has a unique decomposition as

$$
u=v+w,
$$

where $v \in C^{2}(\Omega)$ satisfies $L_{m} v=0$ in $\Omega$ and $w \in C^{2}\left(\mathbb{H}^{+} \backslash K\right)$ satisfies $L_{m} w=0$ in $\mathbb{H}^{+} \backslash K$ with $\lim _{\partial \mathbb{H}^{+}} w=0$.

Proof For $E \subset \mathbb{C}$ and $\rho>0$, define $E_{\rho}=\{x \in \mathbb{C}: d(x, E)<\rho\}$, i.e. $E_{\rho}$ is a neighborhood of $E$.

First, assume that $\Omega$ is a relatively compact open set in $\mathbb{H}^{+}$. Choose $\rho$ small enough so that $K_{\rho}$ and $(\partial \Omega)_{\rho}$ are disjoint. There is a function $\varphi_{\rho} \in \mathcal{D}\left(\mathbb{H}^{+}\right)$compactly supported on $\Omega \backslash K$ such that $\varphi_{\rho} \equiv 1$ in a neighborhood of $\Omega \backslash\left(K_{\rho} \cup(\partial \Omega)_{\rho}\right)$ (Fig. 4).

For $z=x+i y \in \Omega \backslash\left(K_{\rho} \cup(\partial \Omega)_{\rho}\right)$, denote

$$
F_{z}(\zeta)=F_{m}(x, y, \xi, \eta), \quad L_{\zeta}=L_{m, \xi, \eta} \quad \text { for } \quad \zeta=\xi+i \eta
$$




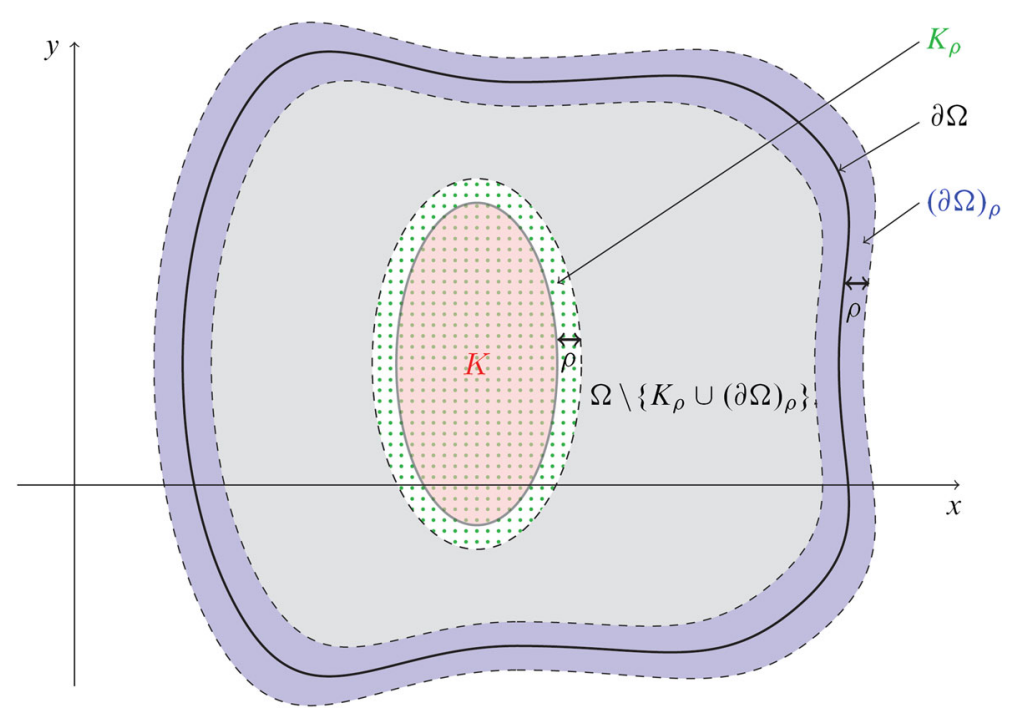

Fig. $4 \varphi_{\rho} \equiv 1$ on the grey domain

Thanks to Proposition 4.7, we have

$$
\begin{aligned}
u(z) & =u \varphi_{\rho}(z)=\int_{\Omega_{\rho}} F_{z}(\zeta) L_{\zeta}\left(u \varphi_{\rho}\right)(\zeta) d \xi d \eta \\
& =\int_{(\partial \Omega)_{\rho}} F_{z}(\zeta) L_{\zeta}\left(u \varphi_{\rho}\right)(\zeta) d \xi d \eta+\int_{K_{\rho}} F_{z}(\zeta) L_{\zeta}\left(u \varphi_{\rho}\right)(\zeta) d \xi d \eta \\
& =v_{\rho}(z)+w_{\rho}(z) .
\end{aligned}
$$

Then, the last result of Proposition 5.7 shows us that $v_{\rho}$ satisfies $L_{m} v_{\rho}=0$ on $\Omega \backslash(\partial \Omega)_{\rho}$ and $w_{\rho}$ satisfies $L_{m} w_{\rho}=0$ on $\mathbb{H}^{+} \backslash K_{\rho}$. We also have $\lim _{\partial \mathbb{H}^{+}} w_{\rho}=0$.

Now, assume that $\sigma<\rho$. As previously, we obtain the decomposition $u=v_{\sigma}+w_{\sigma}$ on $\Omega \backslash\left(K_{\sigma} \cup(\partial \Omega)_{\sigma}\right)$. We claim that $v_{\rho}=v_{\sigma}$ on $\Omega \backslash(\partial \Omega)_{\rho}$ and $w_{\rho}=w_{\sigma}$ on $\mathbb{H}^{+} \backslash K_{\rho}$. To see this, note that if $z \in \Omega \backslash\left(K_{\rho} \cup(\partial \Omega)_{\rho}\right)$, then $v_{\rho}(z)+w_{\rho}(z)=v_{\sigma}(z)+w_{\sigma}(z)$.

The function $w_{\rho}-w_{\sigma}$ satisfies (1) on $\mathbb{H}^{+} \backslash K_{\rho}$, which is equal to $v_{\sigma}-v_{\rho}$ on $\Omega \backslash\left(K_{\rho} \cup(\partial \Omega)_{\rho}\right)$, therefore $v_{\sigma}-v_{\rho}$ extends to a solution of (1) on $\Omega \backslash(\partial \Omega)_{\rho}$. Finally, $w_{\rho}-w_{\sigma}$ extends to a solution of $(1)$ on $\mathbb{H}^{+}$, and $\lim _{\partial \mathbb{H}^{+}}\left(w_{\rho}-w_{\sigma}\right)=0$. Due to Proposition 5.3, we have $w_{\rho}=w_{\sigma}$, and hence $v_{\rho}=v_{\sigma}$.

For $z \in \Omega$, we can define $v(z)=v_{\rho}(z)$ for $\rho$ small enough so that $z \in \Omega \backslash(\partial \Omega)_{\rho}$. Similarly, for $z \in \mathbb{H}^{+} \backslash K$, we put $w(z)=w_{\rho}(z)$ for small $\rho$. Thus we have established the desired decomposition $u=v+w$.

Now, assume that $\Omega$ is an arbitrary domain of $\mathbb{H}^{+}$and let $u$ be a solution of $L_{m} u=0$ on $\Omega \backslash K$. Choose $a \in \mathbb{H}^{+}$and $R$ large enough so that $K \subset D(a, R)$ and $D(a, R)$ is a relatively compact set in $\mathbb{H}^{+}$. Let $\omega=\Omega \cap D(a, R)$. Note that $K$ is a compact set in $\omega$ which is a relatively compact open set in $\mathbb{H}^{+}$and $u$ satisfies (1) on $\omega \backslash K$. Applying 
the results demonstrated for relatively compact open sets, we obtain

$$
u(z)=\widetilde{v}(z)+\widetilde{w}(z)
$$

for $z \in \omega \backslash K$, where $\widetilde{v}$ satisfies (1) on the set $\omega$ and $\widetilde{w}$ satisfies (1) on $\mathbb{H}^{+} \backslash K$ with $\lim _{\partial \mathbb{H}+} \widetilde{w}=0$. Note that $V=u-\widetilde{w}$ satisfies (1) on $\Omega \backslash K$ and $V$ can be extended to a solution of (1) in a neighborhood of $K$ because $V=\widetilde{v}$ on $\omega$. The sum $u=V+\widetilde{w}$ provides with the desired decomposition of $u$.

If we have another decomposition $u=v+w$ with $v \in C^{2}(\Omega), L_{m} v=0$ and with $w \in C^{2}\left(\mathbb{H}^{+} \backslash K\right), L_{m} w=0$ and $\lim _{\partial \mathbb{H}^{+}} w=0$, then we have $V-v=w-\widetilde{w}$ on $\Omega \backslash K$. The function $w-\widetilde{w}$ can be extended on $\mathbb{H}^{+}$to a solution of $L_{m}(w-\widetilde{w})=0$ on $\mathbb{H}^{+}$with $\lim _{\partial \mathbb{H}^{+}}(w-\widetilde{w})=0$. Thanks to Proposition 5.3, we obtain $w=\widetilde{w}$, then $V=v$, which completes the proof of the decomposition theorem.

The following proposition is a Poisson formula for axisymmetric potentials in $\mathbb{H}^{+}$.

Proposition 5.10 Let $m \in \mathbb{C}$ be such that $\operatorname{Re} m<1$ and $u: \mathbb{R} \rightarrow \mathbb{R}$ be a continuous and bounded function. Then there is a unique axisymmetric potential $U \in C^{2}\left(\mathbb{H}^{+}\right)$ such that $\lim _{\|(x, y)\| \rightarrow+\infty} U(x, y)=0$ and for all $y \in \mathbb{R}$,

$$
\lim _{(0, y)} U=u(y)
$$

Moreover, we have for all $(x, y) \in \mathbb{H}^{+}$,

$$
U(x, y)=C_{m} x^{1-m} \int_{-\infty}^{\infty} \frac{u(\eta) d \eta}{\left(x^{2}+(y-\eta)^{2}\right)^{1-m / 2}},
$$

where

$$
C_{m}=\frac{1-m}{2 \pi} \int_{0}^{\pi} \sin ^{1-m} \theta d \theta=\frac{1}{2^{m} \pi} \frac{\Gamma^{2}(1-m / 2)}{\Gamma(1-m)} .
$$

Proof Let us first show that (15) is a solution of $L_{m} U=0$. Let $f(x, y)=x^{1-m} /\left(x^{2}+\right.$ $\left.(y-\eta)^{2}\right)^{1-m / 2}$. Interchanging differentiation and integration, it suffices to prove that $L_{m} f=0$. We have

$$
\begin{aligned}
& \partial_{x} f=\frac{(1-m) x^{-m}}{\left(x^{2}+(y-\eta)^{2}\right)^{1-m / 2}}-\frac{(2-m) x^{2-m}}{\left(x^{2}+(y-\eta)^{2}\right)^{2-m / 2}}, \\
& \partial_{x x} f=-\frac{m(1-m) x^{-m-1}}{\left(x^{2}+(y-\eta)^{2}\right)^{1-m / 2}}-\frac{(2-m)(3-2 m) x^{1-m}}{\left(x^{2}+(y-\eta)^{2}\right)^{2-m / 2}} \\
& +\frac{(2-m)(4-m) x^{3-m}}{\left(x^{2}+(y-\eta)^{2}\right)^{3-m / 2}}, \\
& \partial_{y y} f=-\frac{(2-m) x^{1-m}}{\left(x^{2}+(y-\eta)^{2}\right)^{2-m / 2}}+\frac{(2-m)(4-m)(y-\eta)^{2} x^{1-m}}{\left(x^{2}+(y-\eta)^{2}\right)^{3-m / 2}} \text {. }
\end{aligned}
$$


Then,

$$
\Delta f=\frac{m(2-m) x^{1-m}}{\left(x^{2}+(y-\eta)^{2}\right)^{2-m / 2}}-\frac{m(1-m) x^{-m-1}}{\left(x^{2}+(y-\eta)^{2}\right)^{1-m / 2}}
$$

and we deduce that $L_{m} f(x, y)=0$.

We have

$$
\begin{aligned}
U(x, y) & =C_{m} x^{1-m} \int_{-\infty}^{\infty} \frac{u(\eta) d \eta}{\left(x^{2}+(y-\eta)^{2}\right)^{1-m / 2}} \\
& =\frac{C_{m}}{x} \int_{-\infty}^{\infty} \frac{u(\eta) d \eta}{\left(1+((y-\eta) / x)^{2}\right)^{1-m / 2}} .
\end{aligned}
$$

By a change of variable $t=(y-\eta) / x$, we obtain

$$
U(x, y)=C_{m} \int_{-\infty}^{\infty} \frac{u(y-t x) d t}{\left(1+t^{2}\right)^{1-m / 2}} .
$$

Thanks to the dominated convergence theorem, it suffices to show that

$$
C_{m} \int_{-\infty}^{\infty} \frac{d t}{\left(1+t^{2}\right)^{1-m / 2}}=\frac{1-m}{2 \pi} \int_{0}^{\pi} \sin ^{1-m} \theta d \theta \int_{-\infty}^{\infty} \frac{d t}{\left(1+t^{2}\right)^{1-m / 2}}=1
$$

To see this, according [1, p. 258], note that

$$
\int_{-\infty}^{\infty} \frac{d t}{\left(1+t^{2}\right)^{1-m / 2}}=\mathrm{B}\left(\frac{1}{2}, \frac{1-m}{2}\right)=\frac{\Gamma(1 / 2) \Gamma((1-m) / 2)}{\Gamma(1-m / 2)},
$$

where B is the Euler beta function and

$$
\begin{aligned}
\frac{1-m}{2 \pi} \int_{0}^{\pi} \sin ^{1-m} \theta d \theta & =\frac{1-m}{2 \pi} 2^{1-m} \mathrm{~B}\left(1-\frac{m}{2}, 1-\frac{m}{2}\right) \\
& =\frac{1-m}{2 \pi} 2^{1-m} \frac{\Gamma^{2}(1-m / 2)}{\Gamma(2-m)} .
\end{aligned}
$$

By the duplication formula for the $\Gamma$ function,

$$
\Gamma(2 z)=\pi^{-1 / 2} 2^{2 z-1} \Gamma(z) \Gamma\left(z+\frac{1}{2}\right)
$$

and by the recurrence formula $\Gamma(z+1)=z \Gamma(z)$, we obtain the desired result:

$$
\frac{\Gamma(1 / 2) \Gamma((1-m) / 2)}{\Gamma(1-m / 2)} \frac{1-m}{2 \pi} 2^{1-m} \frac{\Gamma^{2}(1-m / 2)}{\Gamma(2-m)}=1
$$

The uniqueness follows from Proposition 5.3. The proof is complete. 
Remark 5.11 One may ask if there is a reproducing formula for the case $\operatorname{Re} m \geq 1$. Let $m \in \mathbb{N}^{*}$ and let $u \in C^{2}\left(\overline{\mathbb{H}^{+}}\right)$be such that $L_{m} u=0$ on $\mathbb{H}^{+}$, then the function $v$ defined on $\mathbb{R}^{m+2}$ by

$$
v\left(x_{1}, \ldots, x_{m+2}\right)=u\left(\sqrt{x_{1}^{2}+\cdots+x_{m+1}^{2}}, x_{m+2}\right)
$$

is harmonic on $\left(\mathbb{R}^{m+1}\right)_{*} \times \mathbb{R}$. In particular, if $m \geq 2$, by [19, Proposition 18 , p. 310], $v$ can be extended to a harmonic function on $\mathbb{R}^{m+2}$, which tends to 0 at infinity. We then deduce that $v \equiv 0$ hence $u \equiv 0$. This shows that solving $L_{m} u=0$ with $u$ tending to 0 at infinity and with prescribed values of $u$ on the $y$-axis is a problem which does not make sense. In this case, the fact that there is no solution to this Dirichlet problem is a consequence of the loss of ellipticity of $L_{m}$ on the boundary of $\mathbb{H}^{+}$. Therefore, we do not deal with the case $\operatorname{Re} m \geq 1$.

\section{Fourier-Legendre decomposition}

First, we will introduce a specific system of coordinates $(\tau, \theta)$ called bipolar coordinates, see [39]. The numerical applications on extremal bounded problems using this system of coordinates can be found in [25-27].

Let $\alpha>0$. Suppose that there is a positive charge at $A=(-\alpha, 0)$ and a negative charge at $B=(\alpha, 0)$ (the absolute values of the two charges are identical). The potential generated by these charges at a point $M$ is $\ln (M A / M B)$ (modulo a multiplicative constant) (Fig. 5).

Definition 6.1 The coordinates

$$
\tau=\ln \frac{M A}{M B}, \quad \theta=\widehat{A M B}
$$

are called bipolar coordinates.

The bipolar coordinates are related to the Cartesian coordinates by the following formulas:

$$
x=\frac{\alpha \operatorname{sh} \tau}{\operatorname{ch} \tau-\cos \theta}, \quad y=\frac{\alpha \sin \theta}{\operatorname{ch} \tau-\cos \theta}
$$

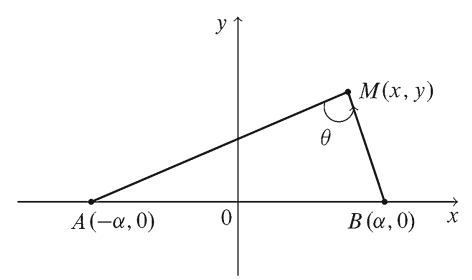

Fig. 5 Bipolar coordinates 
Let $R>0$ and $a=\sqrt{R^{2}+\alpha^{2}}$, the disk with center $(a, 0)$ and radius $R$ is defined in terms of bipolar coordinates as

$$
\tau \geq \tau_{0}=\ln \left(\frac{a}{R}+\sqrt{\frac{a^{2}}{R^{2}}-1}\right)=\operatorname{argch} \frac{a}{R} .
$$

The right half-plane is defined as

$$
\mathbb{H}^{+}=\{(\tau, \theta): \tau \in(0+\infty], \theta \in[0,2 \pi)\} .
$$

The level lines $\tau=\tau_{0}$ are circles with center $\left(\alpha \operatorname{coth} \tau_{0}, 0\right)$ and radii $\alpha / \operatorname{sh} \tau_{0}$. This implies that for all $\tau_{0}, \tau_{1}$ such that $0<\tau_{0}<\tau_{1}$, the set $\left\{(\tau, \theta): \tau \geq \tau_{0}\right\}$ is a closed disk and the set $\left\{(\tau, \theta): 0<\tau<\tau_{1}\right\}$ is the complement in $\mathbb{H}^{+}$of the closed disk $\left\{\tau \geq \tau_{1}\right\}$ (Fig. 6).

The following theorem is well known in physics for $m=-1[3,15,42,45,47,48]$. We extend it to $m \in \mathbb{C}$.

Theorem 6.2 Let $u$ be a solution to $L_{m} u=0$ in an open set in $\mathbb{H}^{+}$. Let

$$
v_{m}(\tau, \theta)=\operatorname{sh}^{(m-1) / 2} \tau(\operatorname{ch} \tau-\cos \theta)^{-m / 2} u(\tau, \theta),
$$

where, by definition,

$$
\operatorname{sh}^{(m-1) / 2} \tau(\operatorname{ch} \tau-\cos \theta)^{-m / 2}=\exp \left(\frac{m-1}{2} \ln \operatorname{sh} \tau-\frac{m}{2} \ln (\operatorname{ch} \tau-\cos \theta)\right)
$$

Then

$$
\frac{\partial^{2} v_{m}}{\partial \tau^{2}}+\frac{\partial^{2} v_{m}}{\partial \theta^{2}}+\operatorname{coth} \tau \frac{\partial v_{m}}{\partial \tau}+\left(\frac{1}{4}-\frac{(m-1)^{2}}{4 \operatorname{sh}^{2} \tau}\right) v_{m}=0
$$

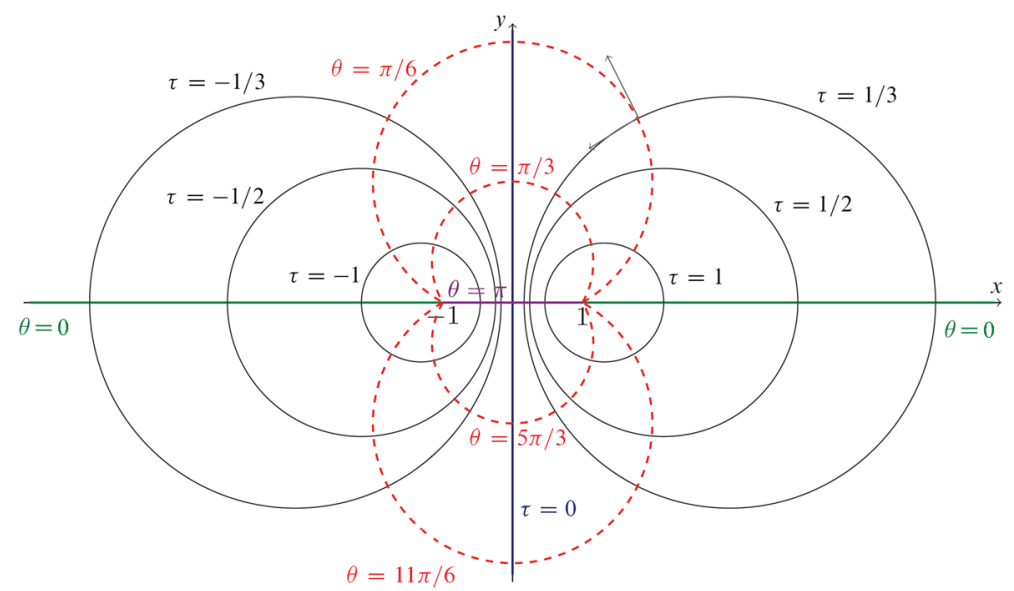

Fig. 6 Level lines (with $\alpha=1$ ) 
Proof We have

$$
\begin{aligned}
& \frac{\partial u}{\partial \tau}=\alpha\left[\frac{1-\operatorname{ch} \tau \cos \theta}{(\operatorname{ch} \tau-\cos \theta)^{2}} \frac{\partial u}{\partial x}-\frac{\operatorname{sh} \tau \sin \theta}{(\operatorname{ch} \tau-\cos \theta)^{2}} \frac{\partial u}{\partial y}\right], \\
& \frac{\partial u}{\partial \theta}=\alpha\left[\frac{-\operatorname{sh} \tau \sin \theta}{(\operatorname{ch} \tau-\cos \theta)^{2}} \frac{\partial u}{\partial x}+\frac{\operatorname{ch} \tau \cos \theta-1}{(\operatorname{ch} \tau-\cos \theta)^{2}} \frac{\partial u}{\partial y}\right] .
\end{aligned}
$$

Thus, we obtain

$$
\frac{\partial u}{\partial x}=\frac{1}{\alpha}\left((1-\operatorname{ch} \tau \cos \theta) \frac{\partial u}{\partial \tau}-\operatorname{sh} \tau \sin \theta \frac{\partial u}{\partial \theta}\right),
$$

and

$$
\begin{aligned}
\frac{\partial^{2} u}{\partial \tau^{2}}=\frac{\alpha^{2}}{(\operatorname{ch} \tau-\cos \theta)^{4}}\left[(1-\operatorname{ch} \tau \cos \theta)^{2} \frac{\partial^{2} u}{\partial x^{2}}\right. & +\operatorname{sh}^{2} \tau \sin ^{2} \theta \frac{\partial^{2} u}{\partial y^{2}} \\
& \left.-2(1-\operatorname{ch} \tau \cos \theta) \operatorname{sh} \tau \sin \theta \frac{\partial^{2} u}{\partial x \partial y}\right] \\
+\frac{\alpha}{(\operatorname{ch} \tau-\cos \theta)^{3}}\left[\operatorname{sh} \tau\left(\cos ^{2} \theta+\operatorname{ch} \tau \cos \theta-2\right) \frac{\partial u}{\partial x}\right. & \left.+\sin \theta\left(\operatorname{ch}^{2} \tau-2+\cos \theta \operatorname{ch} \tau\right) \frac{\partial u}{\partial y}\right]
\end{aligned}
$$

and

$$
\begin{aligned}
& \frac{\partial^{2} u}{\partial \theta^{2}}=\frac{\alpha^{2}}{(\operatorname{ch} \tau-\cos \theta)^{4}}\left[\operatorname{sh}^{2} \tau \sin ^{2} \theta \frac{\partial^{2} u}{\partial x^{2}}\right.+(\operatorname{ch} \tau \cos \theta-1) \frac{\partial^{2} u}{\partial y^{2}} \\
&\left.+2(1-\operatorname{ch} \tau \cos \theta) \operatorname{sh} \tau \sin \theta \frac{\partial^{2} u}{\partial x \partial y}\right] \\
&+\frac{\alpha}{(\operatorname{ch} \tau-\cos \theta)^{3}}\left[\operatorname{sh} \tau\left(2-\cos ^{2} \theta-\cos \theta \operatorname{ch} \tau\right) \frac{\partial u}{\partial x}\right. \\
&\left.+\sin \theta\left(2-\operatorname{ch}^{2} \tau-\operatorname{ch} \tau \cos \theta\right) \frac{\partial u}{\partial y}\right]
\end{aligned}
$$

In particular, we have

$$
\frac{\partial^{2} u}{\partial \tau^{2}}+\frac{\partial^{2} u}{\partial \theta^{2}}=\frac{\alpha^{2}}{(\operatorname{ch} \tau-\cos \theta)^{2}}\left[\frac{\partial^{2} u}{\partial x^{2}}+\frac{\partial^{2} u}{\partial y^{2}}\right] .
$$


Therefore, we obtain

$$
\begin{aligned}
L_{m, x, y} u=\left(\frac{\operatorname{ch} \tau-\cos \theta}{\alpha}\right)^{2}\left(\frac{\partial^{2} u}{\partial \tau^{2}}+\frac{\partial^{2} u}{\partial \theta^{2}}+\right. & \frac{m(1-\operatorname{ch} \tau \cos \theta)}{\operatorname{sh} \tau(\operatorname{ch} \tau-\cos \theta)} \frac{\partial u}{\partial \tau} \\
& \left.-\frac{m \sin \theta}{\operatorname{ch} \tau-\cos \theta} \frac{\partial u}{\partial \theta}\right)
\end{aligned}
$$

According to definition of $v_{m}$,

$$
u(\tau, \theta)=\frac{(\operatorname{ch} \tau-\cos \theta)^{m / 2}}{\operatorname{sh}^{(m-1) / 2} \tau} v_{m}(\tau, \theta)
$$

Denote

$$
r_{m}(\tau, \theta)=\frac{(\operatorname{ch} \tau-\cos \theta)^{m / 2}}{\operatorname{sh}^{(m-1) / 2} \tau}
$$

then

$$
\begin{aligned}
\frac{\partial r_{m}}{\partial \theta} & =\frac{m}{2} \frac{\sin \theta}{\operatorname{ch} \tau-\cos \theta} r_{m} \\
\frac{\partial^{2} r_{m}}{\partial \theta^{2}} & =\frac{m}{4(\operatorname{ch} \tau-\cos \theta)^{2}}\left(2 \cos \theta \operatorname{ch} \tau+m \sin ^{2} \theta-2\right) r_{m}
\end{aligned}
$$

and

$$
\begin{aligned}
\frac{\partial r_{m}}{\partial \tau}=\frac{1}{(\operatorname{ch} \tau-\cos \theta) \operatorname{sh} \tau}\left(\operatorname{ch}^{2} \tau+\right. & (m-1) \operatorname{ch} \tau \cos \theta-m) r_{m} \\
\frac{\partial^{2} r_{m}}{\partial \tau^{2}}=\frac{1}{4(\operatorname{ch} \tau-\cos \theta)^{2} \operatorname{sh}^{2} \tau}\left[\operatorname{ch}^{4} \tau\right. & -2 \operatorname{ch}^{3} \tau \cos \theta+(m-1)^{2} \operatorname{ch}^{2} \tau \cos ^{2} \theta \\
& +2(m-1) \operatorname{ch}^{2} \tau+\left(4-2 m^{2}\right) \operatorname{ch} \tau \cos \theta \\
& \left.+2(m-1) \cos ^{2} \theta+m(m-2)\right] r_{m} .
\end{aligned}
$$

Hence the equation $L_{m, x, y} u=0$ can be rewritten as

$$
\begin{aligned}
r_{m}\left(\frac{\partial^{2} v_{m}}{\partial \tau^{2}}+\frac{\partial^{2} v_{m}}{\partial \theta^{2}}\right) & +\frac{\partial v_{m}}{\partial \tau}\left(2 \frac{\partial r_{m}}{\partial \tau}+\frac{m}{\operatorname{sh} \tau} \frac{1-\operatorname{ch} \tau \cos \theta}{\operatorname{ch} \tau-\cos \theta} r_{m}\right) \\
& +\frac{\partial v_{m}}{\partial \theta}\left(2 \frac{\partial r_{m}}{\partial \theta}-\frac{m \sin \theta}{\operatorname{ch} \tau-\cos \theta} r_{m}\right) \\
& +v_{m}\left(\frac{\partial^{2} r_{m}}{\partial \tau^{2}}+\frac{\partial^{2} r_{m}}{\partial \theta^{2}}+\frac{m(1-\operatorname{ch} \tau \cos \theta)}{\operatorname{sh} \tau(\operatorname{ch} \tau-\cos \theta)} \frac{\partial r_{m}}{\partial \tau}\right. \\
& \left.-\frac{m \sin \theta}{\operatorname{ch} \tau-\cos \theta} \frac{\partial r_{m}}{\partial \theta}\right)=0
\end{aligned}
$$


with

$$
\begin{gathered}
2 \frac{\partial r_{m}}{\partial \tau}+\frac{m}{\operatorname{sh} \tau} \frac{1-\operatorname{ch} \tau \cos \theta}{\operatorname{ch} \tau-\cos \theta} r_{m}=r_{m} \operatorname{coth} \tau, \\
2 \frac{\partial r_{m}}{\partial \theta}-\frac{m \sin \theta}{\operatorname{ch} \tau-\cos \theta} r_{m}=0
\end{gathered}
$$

and

$$
\begin{aligned}
\frac{\partial^{2} r_{m}}{\partial \tau^{2}}+\frac{\partial^{2} r_{m}}{\partial \theta^{2}} & +\frac{m(1-\operatorname{ch} \tau \cos \theta)}{\operatorname{sh} \tau(\operatorname{ch} \tau-\cos \theta)} \frac{\partial r_{m}}{\partial \tau} \\
& -\frac{m \sin \theta}{\operatorname{ch} \tau-\cos \theta} \frac{\partial r_{m}}{\partial \theta}=\left(\frac{1}{4}-\frac{(m-1)^{2}}{4 \operatorname{sh}^{2} \tau}\right) r_{m} .
\end{aligned}
$$

This completes the proof.

Let us apply the method of separation of variables, i.e. assume $v_{m}$ has the form $v_{m}(\tau, \theta)=A_{m}(\tau) B_{m}(\theta)$. Then (16) becomes

$$
\frac{A_{m}^{\prime \prime}}{A_{m}}+\operatorname{coth} \tau \frac{A_{m}^{\prime}}{A_{m}}+\frac{1}{4}-\frac{(m-1)^{2}}{4 \operatorname{sh}^{2} \tau}=-\frac{B_{m}^{\prime \prime}}{B_{m}} .
$$

The term on the right depends only on $\theta$ and the left-hand side depends only on $\tau$, thus we deduce that both are constant. Let $n \in \mathbb{C}$ be such that this constant is equal to $n^{2}$. Then we have

$$
\left\{\begin{array}{l}
A_{m}^{\prime \prime}+\operatorname{coth} \tau A_{m}^{\prime}+\left(\frac{1}{4}-\frac{(m-1)^{2}}{4 \operatorname{sh}^{2} \tau}-n^{2}\right) A_{m}=0 \\
B_{m}^{\prime \prime}+n^{2} B_{m}=0
\end{array}\right.
$$

The function $B_{m}$ is a $2 \pi$-periodic function as $\theta$ represents an angle, therefore $n$ should necessarily be an integer.

To examine the equation satisfied by $A_{m}$, we carry out the following change of function

$$
A_{m}(\tau)=C_{m}(\operatorname{ch} \tau)
$$

Then, $C_{m}$ satisfies

$$
\operatorname{sh}^{2} \tau C_{m}^{\prime \prime}(\operatorname{ch} \tau)+2 \operatorname{ch} \tau C_{m}^{\prime}(\operatorname{ch} \tau)+\left(\frac{1}{4}-n^{2}-\frac{(m-1)^{2}}{4 \operatorname{sh}^{2} \tau}\right) C_{m}(\operatorname{ch} \tau)=0
$$

which can be rewritten as

$$
\begin{aligned}
\left(1-\operatorname{ch}^{2} \tau\right) C_{m}^{\prime \prime}(\operatorname{ch} \tau) & -2 \operatorname{ch} \tau C_{m}^{\prime}(\operatorname{ch} \tau) \\
+ & \left(n^{2}-\frac{1}{4}-\frac{((m-1) / 2)^{2}}{1-\operatorname{ch}^{2} \tau}\right) C_{m}(\operatorname{ch} \tau)=0 .
\end{aligned}
$$


This equation is called the hyperbolic associated Legendre equation. Note that if we put $z=\operatorname{ch} \tau$ and $u(z)=C_{m}(\operatorname{ch} \tau)$, then

$$
\left(1-z^{2}\right) u^{\prime \prime}-2 z u^{\prime}+\left[v(v+1)-\frac{\mu^{2}}{1-z^{2}}\right] u=0
$$

where

$$
v=n-\frac{1}{2}, \quad \mu=\frac{m-1}{2}
$$

This equation is called the associated Legendre equation, and it can be reduced to the Legendre equation if $\mu=0$ :

$$
\left(1-z^{2}\right) u^{\prime \prime}-2 z u^{\prime}+v(v+1) u=0 .
$$

Two independent solutions of (LA) are given in Appendix, where they are denoted by $P_{\nu}^{\mu}(\operatorname{ch} \tau)$ and $Q_{\nu}^{\mu}(\operatorname{ch} \tau)$.

Theorem 6.3 Let $m \in \mathbb{C}$ and $0<\tau_{0}$. Let $u$ be a smooth solution to $L_{m} u=0$ on the disk $\tau \geq \tau_{0}$ and let $v$ be a smooth solution to $L_{m} v=0$ on $\mathbb{H}^{+} \backslash\left\{\tau>\tau_{0}\right\}$, which is the complement in $\mathbb{H}^{+}$of the disk $\left\{\tau>\tau_{0}\right\}$, and assume that $\lim _{\partial \mathbb{H}^{+}} v=0$. Then there are two sequences $\left(a_{n}\right)_{n \in \mathbb{Z}}$ and $\left(b_{n}\right)_{n \in \mathbb{Z}}$ of $\ell^{2}(\mathbb{Z})$ (rapidly decreasing) such that

$$
\begin{aligned}
& u=\sum_{n=-\infty}^{+\infty} a_{n} Q_{n-1 / 2}^{(m-1) / 2}(\operatorname{ch} \tau) \operatorname{sh}^{(1-m) / 2} \tau(\operatorname{ch} \tau-\cos \theta)^{m / 2} e^{i n \theta}, \\
& v=\sum_{n=-\infty}^{+\infty} b_{n} P_{n-1 / 2}^{(m-1) / 2}(\operatorname{ch} \tau) \operatorname{sh}^{(1-m) / 2} \tau(\operatorname{ch} \tau-\cos \theta)^{m / 2} e^{i n \theta} .
\end{aligned}
$$

The sequence $\left(a_{n}\right)$ is unique. In addition, the convergence of the first series is uniform on every compact set $\left[\tau_{1}, \tau_{2}\right]$ of the disk $\tau>\tau_{0}$ with $\tau_{0} \leq \tau_{1}<\tau_{2}$. And the convergence of the second one is uniform on every compact set $\left[\tau_{3}, \tau_{4}\right]$ of the complement of the disk $\tau>\tau_{0}$ in $\mathbb{H}^{+}$with $0<\tau_{3}<\tau_{4} \leq \tau_{0}$.

If $\operatorname{Re} m<1$, then the sequence $\left(b_{n}\right)$ is unique.

Proof Indeed, decomposing the function

$$
\theta \mapsto u\left(\tau_{0}, \theta\right)\left(\operatorname{ch} \tau_{0}-\cos \theta\right)^{-m / 2} \operatorname{sh}^{(m-1) / 2} \tau_{0}
$$

as Fourier series with respect to the variable $\theta$, yields the following Fourier expansion for $u\left(\tau_{0}, \cdot\right)$ :

$$
u\left(\tau_{0}, \theta\right)=\operatorname{sh}^{(1-m) / 2} \tau_{0}\left(\operatorname{ch} \tau_{0}-\cos \theta\right)^{m / 2} \sum_{n=-\infty}^{+\infty} a_{n} e^{i n \theta}
$$


where $a_{n} \in \ell^{2}(\mathbb{Z})$ satisfies

$$
a_{n}=\frac{1}{2 \pi} \int_{0}^{2 \pi}\left(\operatorname{ch} \tau_{0}-\cos \theta\right)^{-m / 2} \operatorname{sh}^{(m-1) / 2} \tau_{0} u\left(\tau_{0}, s\right) e^{-i n s} d s .
$$

The function $u\left(\tau_{0}, \cdot\right)$ is a smooth function of the variable $\theta$, therefore the sequence $\left(a_{n}\right)_{n}$ is rapidly decreasing as $|n| \rightarrow+\infty$.

The function

$$
\widetilde{u}(\tau, \theta)=\operatorname{sh}^{(1-m) / 2} \tau(\operatorname{ch} \tau-\cos \theta)^{m / 2} \sum_{n=-\infty}^{+\infty} a_{n} \frac{Q_{n-1 / 2}^{(m-1) / 2}(\operatorname{ch} \tau)}{Q_{n-1 / 2}^{(m-1) / 2}\left(\operatorname{ch} \tau_{0}\right)} e^{i n \theta}
$$

coincides with $u$ on the circle $\tau=\tau_{0}$. Let us see that $\tilde{u}$ is well defined on the disk $\tau \geq \tau_{0}$. Indeed, thanks to Proposition 8.1, as $|n| \rightarrow+\infty$,

$$
\frac{Q_{n-1 / 2}^{(m-1) / 2}(\operatorname{ch} \tau)}{Q_{n-1 / 2}^{(m-1) / 2}\left(\operatorname{ch} \tau_{0}\right)} \sim \sqrt{\frac{\operatorname{sh} \tau_{0}}{\operatorname{sh} \tau}} e^{|n|\left(\tau_{0}-\tau\right)}
$$

and this equivalence is uniform on all compact sets $\left[\tau_{1}, \tau_{2}\right]$ with $0<\tau_{0} \leq \tau_{1}<\tau_{2}$. It follows that the series which defines $\tilde{u}$ is norm convergent on any compact set $\left[\tau_{1}, \tau_{2}\right]$ of the disk $\tau \geq \tau_{0}$. The same is true for derivatives with respect to $\tau$ and $\theta$ (which are also expressed through the associated Legendre functions, see Appendix).

Due to the fact that the solution of an elliptic equation is uniquely determined by its boundary values (this follows from the maximum principle), we deduce that $\tilde{u}$ is the unique axisymmetric potential on the disk $\tau \geq \tau_{0}$ which coincides with $u$ on the circle $\tau=\tau_{0}$.

For $v$, the proof is similar. Indeed, decomposing the function

$$
\theta \mapsto v\left(\tau_{0}, \theta\right)\left(\operatorname{ch} \tau_{0}-\cos \theta\right)^{-m / 2} \operatorname{sh}^{(m-1) / 2} \tau_{0}
$$

as Fourier series with respect to the variable $\theta$, yields the following Fourier expansion for $v\left(\tau_{0}, \cdot\right)$ :

$$
v\left(\tau_{0}, \theta\right)=\operatorname{sh}^{(1-m) / 2} \tau_{0}\left(\operatorname{ch} \tau_{0}-\cos \theta\right)^{m / 2} \sum_{n=-\infty}^{+\infty} b_{n} e^{i n \theta}
$$

where $b_{n} \in \ell^{2}(\mathbb{Z})$ satisfies

$$
b_{n}=\frac{1}{2 \pi} \int_{0}^{2 \pi}\left(\operatorname{ch} \tau_{0}-\cos \theta\right)^{-m / 2} \operatorname{sh}^{(m-1) / 2} \tau_{0} v\left(\tau_{0}, s\right) e^{-i n s} d s .
$$

The function $v\left(\tau_{0}, \cdot\right)$ is a smooth function of the variable $\theta$, therefore the sequence $\left(b_{n}\right)_{n}$ is rapidly decreasing as $|n| \rightarrow+\infty$. 
The function

$$
\widetilde{v}(\tau, \theta)=\operatorname{sh}^{(1-m) / 2} \tau(\operatorname{ch} \tau-\cos \theta)^{m / 2} \sum_{n=-\infty}^{+\infty} b_{n} \frac{P_{n-1 / 2}^{(m-1) / 2}(\operatorname{ch} \tau)}{P_{n-1 / 2}^{(m-1) / 2}\left(\operatorname{ch} \tau_{0}\right)} e^{i n \theta}
$$

coincides with $v$ on the circle $\tau=\tau_{0}$. Let us see that $\widetilde{v}$ is well defined on the complement of the disk $\tau>\tau_{0}$. Indeed, thanks to Proposition 8.1, as $|n| \rightarrow+\infty$,

$$
\frac{P_{n-1 / 2}^{(m-1) / 2}(\operatorname{ch} \tau)}{P_{n-1 / 2}^{(m-1) / 2}\left(\operatorname{ch} \tau_{0}\right)} \sim \sqrt{\frac{\operatorname{sh} \tau_{0}}{\operatorname{sh} \tau}} e^{|n|\left(\tau-\tau_{0}\right)}
$$

and this equivalence is uniform on all compact sets $\left[\tau_{1}, \tau_{2}\right]$ with $0<\tau_{1}<\tau_{2} \leq \tau_{0}$. It follows that the series which defines $\widetilde{v}$ is norm convergent on any compact set $\left[\tau_{1}, \tau_{2}\right]$ of the complement of the disc $\tau>\tau_{0}$. The same is true for derivatives with respect to $\tau$ and $\theta$.

We will show that

$$
\lim _{\tau \rightarrow 0+} \widetilde{v}=0
$$

If $\operatorname{Re} m<1$, by formula (18), for $n \in \mathbb{N}$ we have

$$
\begin{gathered}
P_{n-1 / 2}^{(m-1) / 2}(\operatorname{ch} \tau)=\frac{2^{(m-1) / 2}}{\sqrt{\pi} \Gamma(1-m / 2)} \operatorname{sh}^{(1-m) / 2} \tau \\
\int_{0}^{\pi}(\operatorname{ch} \tau+\operatorname{sh} \tau \cos \theta)^{n+m / 2-1} \sin ^{1-m} \theta d \theta,
\end{gathered}
$$

hence

$$
\lim _{\tau \rightarrow 0+} P_{n-1 / 2}^{(m-1) / 2}(\operatorname{ch} \tau)=0
$$

In addition, for $n>1-\operatorname{Re} m / 2$, we have

$$
\begin{aligned}
\left|P_{n-1 / 2}^{(m-1) / 2}(\operatorname{ch} \tau)\right| & \frac{2^{(\operatorname{Re} m-1) / 2} \operatorname{sh}^{(1-\operatorname{Re} m) / 2} \tau}{\sqrt{\pi}|\Gamma(1-m / 2)|} \\
& \int_{0}^{\pi}(\operatorname{ch} \tau+\operatorname{sh} \tau \cos \theta)^{n+\operatorname{Re} m / 2-1} \sin ^{1-\operatorname{Re} m} \theta d \theta \\
& \leq \frac{2^{(\operatorname{Re} m-1) / 2} \operatorname{sh}^{(1-\operatorname{Re} m) / 2} \tau}{\sqrt{\pi}|\Gamma(1-m / 2)|} \\
& \int_{0}^{\pi}(\operatorname{ch} \tau+\operatorname{sh} \tau)^{n+\operatorname{Re} m / 2-1} \sin ^{1-\operatorname{Re} m} \theta d \theta \\
& \leq C_{m} \operatorname{sh}^{(1-\operatorname{Re} m) / 2} \tau e^{(n+\operatorname{Re} m / 2) \tau}
\end{aligned}
$$


thus

$$
\sum_{n>1-\operatorname{Re} m / 2} \sup _{\tau \in\left[0, \tau_{0} / 2\right]}\left|b_{n} \frac{P_{n-1 / 2}^{(m-1) / 2}(\operatorname{ch} \tau)}{P_{n-1 / 2}^{(m-1) / 2}\left(\operatorname{ch} \tau_{0}\right)} e^{i n \theta}\right|<+\infty
$$

By Proposition 8.1, we obtain

$$
P_{n-1 / 2}^{(m-1) / 2}\left(\operatorname{ch} \tau_{0}\right) \sim \frac{n^{m / 2-1}}{\sqrt{2 \pi \operatorname{sh} \tau_{0}}} e^{n \tau_{0}} \quad \text { as } n \rightarrow+\infty .
$$

So, $\lim _{\tau \rightarrow 0+} \widetilde{v}=0$.

The uniqueness of $\left(b_{n}\right)$ for $\operatorname{Re} m<1$ follows from the following fact established in the next section: the families

$$
\begin{aligned}
& \mathcal{A}=\left(\frac{Q_{n-1 / 2}^{(m-1) / 2}(\operatorname{ch} \tau)}{Q_{n-1 / 2}^{(m-1) / 2}\left(\operatorname{ch} \tau_{0}\right)} \frac{(\operatorname{ch} \tau-\cos \theta)^{m / 2}}{\operatorname{sh}^{(m-1) / 2} \tau} e^{i n \theta}\right)_{n \in \mathbb{Z}}=\left(a_{n}\right)_{n \in \mathbb{Z}}, \\
& \mathcal{B}=\left(\frac{P_{n-1 / 2}^{(m-1) / 2}(\operatorname{ch} \tau)}{P_{n-1 / 2}^{(m-1) / 2}\left(\operatorname{ch} \tau_{1}\right)} \frac{(\operatorname{ch} \tau-\cos \theta)^{m / 2}}{\operatorname{sh}^{(m-1) / 2} \tau} e^{i n \theta}\right)_{n \in \mathbb{Z}}=\left(b_{n}\right)_{n \in \mathbb{Z}}
\end{aligned}
$$

form a Riesz basis.

Corollary 6.4 The solution of the Dirichlet problem for $L_{m} u=0$ on $D((a, 0), R)$, with $u=\varphi$ on $\partial D((a, 0), R)$, is given by

$$
u(\tau, \theta)=\operatorname{sh}^{(1-m) / 2} \tau(\operatorname{ch} \tau-\cos \theta)^{m / 2} \sum_{n=-\infty}^{+\infty} c_{n} \frac{Q_{n-1 / 2}^{(m-1) / 2}(\operatorname{ch} \tau)}{Q_{n-1 / 2}^{(m-1) / 2}\left(\operatorname{ch} \tau_{0}\right)} e^{i n \theta}
$$

where $\left\{\tau=\tau_{0}\right\}$ corresponds to the circle with center $(a, 0)$ and radius $R$ and

$$
c_{n}=\frac{1}{2 \pi} \int_{0}^{2 \pi}\left(\operatorname{ch} \tau_{0}-\cos \theta\right)^{-m / 2} \operatorname{sh}^{(m-1) / 2} \tau_{0} \varphi(a+R \cos s, R \sin s) e^{-i n s} d s .
$$

Similarly, the function

$$
\begin{gathered}
v(\tau, \theta)=\operatorname{sh}^{(1-m) / 2} \tau(\operatorname{ch} \tau-\cos \theta)^{m / 2} \sum_{n=-\infty}^{+\infty} c_{n} \frac{P_{n-1 / 2}^{(m-1) / 2}(\operatorname{ch} \tau)}{P_{n-1 / 2}^{(m-1) / 2}\left(\operatorname{ch} \tau_{0}\right)} e^{i n \theta}, \\
c_{n}=\frac{1}{2 \pi} \int_{0}^{2 \pi}\left(\operatorname{ch} \tau_{0}-\cos \theta\right)^{-m / 2} \operatorname{sh}^{(m-1) / 2} \tau_{0} \varphi(a+R \cos s, R \sin s) e^{-i n s} d s,
\end{gathered}
$$

is a solution to $L_{m} v=0$ on $\mathbb{H}^{+} \backslash D((a, 0), R)$, which is equal to $\varphi$ on $\partial D((a, 0), R)$.

Moreover, if $\operatorname{Re} m<1$, then $v$ satisfies $\lim _{\partial \mathbb{H}^{+}} v=0$, and (17) is the unique solution of the Dirichlet problem $L_{m} v=0$ on $\mathbb{H}^{+} \backslash D((a, 0), R)$ which vanishes on $\partial \mathbb{H}^{+}$. 


\section{Riesz basis}

We will prove that the first group of functions of the following family:

$$
\left(\frac{(\operatorname{ch} \tau-\cos \theta)^{m / 2}}{\operatorname{sh}^{(m-1) / 2} \tau}\left\{\begin{array}{c}
\cos (n \theta) \\
\sin (n \theta)
\end{array}\right\}\left\{\begin{array}{l}
P_{n-1 / 2}^{(m-1) / 2}(\operatorname{ch} \tau) \\
Q_{n-1 / 2}^{(m-1) / 2}(\operatorname{ch} \tau)
\end{array}\right\}\right)_{n \in \mathbb{Z}}
$$

is a basis of solutions on the disk $\tau \geq \tau_{1}$ and the second one is a basis of solutions on $\tau \leq \tau_{0}$, which is the complement on $\mathbb{H}^{+}$of some disk, with $0<\tau_{0}<\tau_{1}$. This fact is known for $m=-1$, namely, for $\mu=1$. We extend this result for complex $m$.

Let us recall the definition of a Riesz basis. The sequence $\left(x_{n}\right)_{n \in \mathbb{N}}$ is called a quasiorthogonal or Riesz sequence of a Hilbert space $X$ if there are two constants $c, C>0$ such that for all sequences $\left(a_{n}\right)_{n \in \mathbb{Z}}$ in $\ell^{2}$, we have

$$
c^{2} \sum_{n}\left|a_{n}\right|^{2} \leq\left\|\sum_{n} a_{n} x_{n}\right\|^{2} \leq C^{2} \sum_{n}\left|a_{n}\right|^{2} .
$$

If the family $\left(x_{n}\right)_{n \in \mathbb{Z}}$ is complete, it is called a Riesz basis. The matrix of scalar products $\left\{\left\langle x_{i}, x_{j}\right\rangle\right\}_{i, j}$ is called the Gram matrix associated to $\left\{x_{i}\right\}_{i}$. Let us recall the following characterization of a Riesz basis by the Gram matrix.

Property ([43, p. 170]) A family $\left\{x_{i}\right\}_{i}$ is a Riesz basis for some Hilbert space $X$ if $\left\{x_{i}\right\}_{i}$ is complete in $X$ and the Gram matrix associated to $\left\{x_{i}\right\}_{i}$ defines a bounded and invertible operator on $\ell^{2}(\mathbb{N})$.

Let $\mathcal{A}$ and $\mathcal{B}$ be the families of solutions to $L_{m} u=0$, respectively, inside the disk $\tau>\tau_{0}$ and outside the disk $\tau>\tau_{1}$, with $0<\tau_{0}<\tau_{1}$ :

$$
\begin{aligned}
& \mathcal{A}=\left(\frac{Q_{n-1 / 2}^{(m-1) / 2}(\operatorname{ch} \tau)}{Q_{n-1 / 2}^{(m-1) / 2}\left(\operatorname{ch} \tau_{0}\right)} \frac{(\operatorname{ch} \tau-\cos \theta)^{m / 2}}{\operatorname{sh}^{(m-1) / 2} \tau} e^{i n \theta}\right)_{n \in \mathbb{Z}}=\left(a_{n}\right)_{n \in \mathbb{Z}}, \\
& \mathcal{B}=\left(\frac{P_{n-1 / 2}^{(m-1) / 2}(\operatorname{ch} \tau)}{P_{n-1 / 2}^{(m-1) / 2}\left(\operatorname{ch} \tau_{1}\right)} \frac{(\operatorname{ch} \tau-\cos \theta)^{m / 2}}{\operatorname{sh}^{(m-1) / 2} \tau} e^{i n \theta}\right)_{n \in \mathbb{Z}}=\left(b_{n}\right)_{n \in \mathbb{Z}} .
\end{aligned}
$$

Let $\mathcal{C}$ be the union of these two families,

$$
\mathcal{C}=\left(c_{n}\right)_{n \in \mathbb{Z}}=\left(c_{2 n}=a_{n} \text { and } c_{2 n+1}=b_{n}\right)_{n \in \mathbb{Z}} .
$$

Denote the annulus defined in terms of bipolar coordinates $\left\{0<\tau_{0}<\tau<\tau_{1}\right\}$ by $\mathbb{A}$. The space $L^{2}(\partial \mathbb{A})$ is equipped with the following inner product: for $f, g \in L^{2}(\partial \mathbb{A})$,

$$
\begin{aligned}
\langle f, g\rangle=\frac{1}{2 \pi} \int_{0}^{2 \pi} f\left(\tau_{0}, \theta\right) \overline{g\left(\tau_{0}, \theta\right)} \frac{\operatorname{sh}^{\operatorname{Re} m-1} \tau_{0}}{\left(\operatorname{ch} \tau_{0}-\cos \theta\right)^{\operatorname{Re} m}} d \theta \\
\quad+\frac{1}{2 \pi} \int_{0}^{2 \pi} f\left(\tau_{1}, \theta\right) \overline{g\left(\tau_{1}, \theta\right)} \frac{\operatorname{sh}^{\operatorname{Re} m-1} \tau_{1}}{\left(\operatorname{ch} \tau_{1}-\cos \theta\right)^{\operatorname{Re} m}} d \theta .
\end{aligned}
$$


Proposition 7.1 $\mathcal{C}$ is a Riesz basis in the Hilbert space $L^{2}(\partial \mathbb{A})$.

Proof To find the Gram matrix for $\mathcal{C}$, first calculate all its elements. For $n \in \mathbb{Z}$,

$$
\begin{aligned}
\left\langle c_{2 n}, c_{2 n}\right\rangle & =1+\left|\frac{Q_{n-1 / 2}^{(m-1) / 2}\left(\mathrm{ch} \tau_{1}\right)}{Q_{n-1 / 2}^{(m-1) / 2}\left(\operatorname{ch} \tau_{0}\right)}\right|^{2}, \\
\left\langle c_{2 n+1}, c_{2 n+1}\right\rangle & =1+\left|\frac{P_{n-1 / 2}^{(m-1) / 2}\left(\operatorname{ch} \tau_{0}\right)}{P_{n-1 / 2}^{(m-1) / 2}\left(\operatorname{ch} \tau_{1}\right)}\right|^{2}, \\
\left\langle c_{2 n}, c_{2 n+1}\right\rangle & =\frac{P_{n-1 / 2}^{(m-1) / 2}\left(\operatorname{ch} \tau_{0}\right)}{P_{n-1 / 2}^{(m-1) / 2}\left(\operatorname{ch} \tau_{1}\right)}+\frac{Q_{n-1 / 2}^{(m-1) / 2}\left(\operatorname{ch} \tau_{1}\right)}{Q_{n-1 / 2}^{(m-1) / 2}\left(\operatorname{ch} \tau_{0}\right)}, \\
\left\langle c_{2 n+1}, c_{2 n}\right\rangle & =\overline{\left\langle c_{2 n}, c_{2 n+1}\right\rangle} .
\end{aligned}
$$

In all other cases, the inner product is zero, hence the Gram matrix is diagonal by blocks and each block is the following $2 \times 2$ matrix:

$$
M_{n}=\left(\begin{array}{cc}
1+\left|\frac{Q_{n-1 / 2}^{(m-1) / 2}\left(\operatorname{ch} \tau_{1}\right)}{Q_{n-1 / 2}^{(m-1) / 2}\left(\operatorname{ch} \tau_{0}\right)}\right|^{2} & \frac{P_{n-1 / 2}^{(m-1) / 2}\left(\operatorname{ch} \tau_{0}\right)}{P_{n-1 / 2}^{(m-1) / 2}\left(\operatorname{ch} \tau_{1}\right)}+\frac{Q_{n-1 / 2}^{(m-1) / 2}\left(\operatorname{ch} \tau_{1}\right)}{Q_{n-1 / 2}^{(m-1) / 2}\left(\operatorname{ch} \tau_{0}\right)} \\
\frac{P_{n-1 / 2}^{(m-1) / 2}\left(\operatorname{ch} \tau_{0}\right)}{P_{n-1 / 2}^{(m-1) / 2}\left(\operatorname{ch} \tau_{1}\right)}+\overline{\frac{Q_{n-1 / 2}^{(m-1) / 2}\left(\operatorname{ch} \tau_{1}\right)}{Q_{n-1 / 2}^{(m-1) / 2}\left(\operatorname{ch} \tau_{0}\right)}} & 1+\left|\frac{P_{n-1 / 2}^{(m-1) / 2}\left(\operatorname{ch} \tau_{0}\right)}{P_{n-1 / 2}^{(m-1) / 2}\left(\operatorname{ch} \tau_{1}\right)}\right|^{2}
\end{array}\right) .
$$

The Gram matrix $G$ has the form

$$
G=\left(\begin{array}{cccccccc}
M_{0} & 0 & \ldots & \ldots & \ldots & \ldots & \ldots & \ldots \\
0 & M_{-1} & 0 & \ldots & \ldots & \ldots & \ldots & \ldots \\
\vdots & 0 & M_{1} & 0 & \ldots & \ldots & \ldots & \ldots \\
\vdots & \ddots & 0 & M_{-2} & 0 & \ldots & \ldots & \ldots \\
\vdots & \ddots & \ddots & 0 & \ddots & \ddots & \ldots & \ldots \\
\vdots & \ddots & \ddots & \ddots & \ddots & M_{-n} & \ddots & \ldots \\
\vdots & \ddots & \ddots & \ddots & \ddots & \ddots & M_{n} & \ddots \\
\vdots & \ddots & \ddots & \ddots & \ddots & \ddots & \ddots & \ddots
\end{array}\right) .
$$

The determinant of $M_{n}$ is

$$
\operatorname{det} M_{n}=\left|1-\frac{Q_{n-1 / 2}^{(m-1) / 2}\left(\operatorname{ch} \tau_{1}\right)}{Q_{n-1 / 2}^{(m-1) / 2}\left(\operatorname{ch} \tau_{0}\right)} \frac{P_{n-1 / 2}^{(m-1) / 2}\left(\operatorname{ch} \tau_{0}\right)}{P_{n-1 / 2}^{(m-1) / 2}\left(\operatorname{ch~} \tau_{1}\right)}\right|^{2}
$$


Let us show that $M_{n}$ is invertible. Suppose the contrary, then det $M_{n}=0$, which is equivalent to

$$
Q_{n-1 / 2}^{(m-1) / 2}\left(\operatorname{ch} \tau_{1}\right) P_{n-1 / 2}^{(m-1) / 2}\left(\operatorname{ch} \tau_{0}\right)=Q_{n-1 / 2}^{(m-1) / 2}\left(\operatorname{ch} \tau_{0}\right) P_{n-1 / 2}^{(m-1) / 2}\left(\operatorname{ch} \tau_{1}\right) .
$$

It can be rewritten as

$$
\left|\begin{array}{ll}
Q_{n-1 / 2}^{(m-1) / 2}\left(\operatorname{ch} \tau_{0}\right) & P_{n-1 / 2}^{(m-1) / 2}\left(\operatorname{ch} \tau_{0}\right) \\
Q_{n-1 / 2}^{(m-1) / 2}\left(\operatorname{ch~} \tau_{1}\right) & P_{n-1 / 2}^{(m-1) / 2}\left(\operatorname{ch~} \tau_{1}\right)
\end{array}\right|=0
$$

with $P_{n-1 / 2}^{(m-1) / 2}\left(\operatorname{ch} \tau_{0}\right), Q_{n-1 / 2}^{(m-1) / 2}\left(\operatorname{ch} \tau_{1}\right) \neq 0$. Therefore, there is $\lambda \in \mathbb{C} \backslash\{0\}$ (which depends on $m, n, \tau_{0}$ and $\tau_{1}$ ) such that

$$
\left\{\begin{array}{l}
Q_{n-1 / 2}^{(m-1) / 2}\left(\operatorname{ch} \tau_{0}\right)=\lambda P_{n-1 / 2}^{(m-1) / 2}\left(\operatorname{ch} \tau_{0}\right) \\
Q_{n-1 / 2}^{(m-1) / 2}\left(\operatorname{ch~} \tau_{1}\right)=\lambda P_{n-1 / 2}^{(m-1) / 2}\left(\operatorname{ch~} \tau_{1}\right) .
\end{array}\right.
$$

Then, by the asymptotic behavior of associated Legendre functions (see Proposition 8.1 in Appendix), on the one hand, we have

$$
\lambda \sim \pi e^{i \pi(m-1) / 2} e^{-2 n \tau_{0}} \quad \text { as } n \rightarrow+\infty
$$

and on the other hand, we have

$$
\lambda \sim \pi e^{i \pi(m-1) / 2} e^{-2 n \tau_{1}} \quad \text { as } n \rightarrow+\infty
$$

what implies that $\tau_{0}=\tau_{1}$, whereas it is not possible. Hence the matrix $M_{n}$ is invertible and this completes the proof.

\section{Appendix: Associated Legendre functions of first and second kind}

In this section, we provide formulas of integral representation for the associated Legendre functions of the first and the second kind with $z=\operatorname{ch} \tau>1$, see $[2,39,50]$.

$$
P_{\nu}^{\mu}(\operatorname{ch} \tau)=\frac{2^{-v} \operatorname{sh}^{-\mu} \tau}{\Gamma(-\mu-v) \Gamma(v+1)} \int_{0}^{\infty}(\operatorname{ch} \tau+\operatorname{ch} \theta)^{\mu-v-1} \operatorname{sh}^{2 \nu+1} \theta d \theta
$$

with $\operatorname{Re} v>-1$ and $\operatorname{Re}(\mu+v)<0$.

$$
P_{\nu}^{\mu}(\operatorname{ch} \tau)=\frac{2^{\mu} \operatorname{sh}^{-\mu} \tau}{\sqrt{\pi} \Gamma(1 / 2-\mu)} \int_{0}^{\pi} \frac{(\operatorname{ch} \tau+\operatorname{sh} \tau \cos \theta)^{\mu+v}}{\sin ^{2 \mu} \theta} d \theta
$$


with $\operatorname{Re} \mu<1 / 2$.

$$
P_{\nu}^{\mu}(\operatorname{ch} \tau)=\sqrt{\frac{2}{\pi}} \frac{\operatorname{sh}^{\mu} \tau}{\Gamma(1 / 2-\mu)} \int_{0}^{\tau} \frac{\operatorname{ch}[(\nu+1 / 2) \theta]}{(\operatorname{ch} \tau-\operatorname{ch} \theta)^{\mu+1 / 2}} d \theta
$$

with $\operatorname{Re} \mu<1 / 2$.

$$
Q_{\nu}^{\mu}(\operatorname{ch} \tau)=\frac{e^{i \pi \mu} \sqrt{\pi}}{2^{\mu}} \frac{\operatorname{sh}^{\mu} \tau \Gamma(\nu+\mu+1)}{\Gamma(v-\mu+1) \Gamma(\mu+1 / 2)} \int_{0}^{\infty} \frac{\operatorname{sh}^{2 \mu} \theta}{(\operatorname{ch} \tau+\operatorname{sh} \tau \operatorname{ch} \theta)^{v+\mu+1}} d \theta
$$

with $\operatorname{Re} \mu>-1 / 2, \operatorname{Re}(v-\mu+1)<0$ and $\operatorname{Re}(v+\mu+1)>0$.

$$
Q_{\nu}^{\mu}(\operatorname{ch} \tau)=\sqrt{\frac{\pi}{2}} e^{i \pi \mu} \frac{\operatorname{sh}^{\mu} \tau}{\Gamma(1 / 2-\mu)} \int_{\tau}^{\infty} \frac{e^{-(\nu+1 / 2) \theta}}{(\operatorname{ch} \theta-\operatorname{ch} \tau)^{\mu+1 / 2}} d \theta
$$

with $\operatorname{Re} \mu<1 / 2$ and $\operatorname{Re}(\mu+v+1)>0$.

$$
Q_{v}^{\mu}(\operatorname{ch} \tau)=e^{i \pi \mu} 2^{-v-1} \frac{\Gamma(v+\mu+1)}{\Gamma(v+1)} \operatorname{sh}^{-\mu} \tau \int_{0}^{\pi}(\operatorname{ch} \tau+\cos \theta)^{\mu-\nu-1} \sin ^{2 v+1} \theta d \theta
$$

with $\operatorname{Re} v>-1$ and $\operatorname{Re}(\mu+v+1)>0$, see [50, pp. 4-6].

There are the following relations between the Legendre functions, see [50, p.6] and [2, Formula 8.2.2]:

$$
\begin{aligned}
P_{\nu}^{\mu} & =P_{-\nu-1}^{\mu}, \\
Q_{-\nu-1}^{\mu}(z) & =\frac{-\pi e^{i \pi \mu} \cos (\pi \nu) P_{\nu}^{\mu}+\sin [\pi(\nu+\mu)] Q_{\nu}^{\mu}}{\sin [\pi(\nu-\mu)]}
\end{aligned}
$$

for $v-\mu \notin \mathbb{Z}$. In particular, for $v=n-1 / 2$ and $n \in \mathbb{Z}$, we have

$$
\begin{aligned}
Q_{-v-1}^{\mu} & =Q_{v}^{\mu}, \quad \mu \in \mathbb{C} \\
e^{i \pi \mu} \Gamma(v+\mu+1) Q_{v}^{-\mu} & =e^{-i \pi \mu} \Gamma(v-\mu+1) Q_{v}^{\mu} \\
P_{v}^{-\mu} & =\frac{\Gamma(v-\mu+1)}{\Gamma(v+\mu+1)}\left[P_{v}^{\mu}-\frac{2}{\pi} e^{-i \pi \mu} \sin (\pi \mu) Q_{v}^{\mu}\right] .
\end{aligned}
$$

There hold the following Whipple formulas relating the associated Legendre functions of first and second kind, see [50, p.6]:

$$
\begin{aligned}
& Q_{\nu}^{\mu}(\operatorname{ch} \tau)=e^{i \pi \mu} \sqrt{\frac{\pi}{2}} \frac{\Gamma(\mu+v+1)}{\sqrt{\operatorname{sh} \tau}} P_{-\mu-1 / 2}^{-\nu-1 / 2}(\operatorname{coth} \tau), \\
& P_{\nu}^{\mu}(\operatorname{ch} \tau)=\frac{i e^{i \pi v}}{\Gamma(-v-\mu)} \sqrt{\frac{2}{\pi}} \frac{1}{\sqrt{\operatorname{sh} \tau}} Q_{-\mu-1 / 2}^{-\nu-1 / 2}(\operatorname{coth} \tau) .
\end{aligned}
$$


There hold the following recursion formulas, see [50, pp.6-7]:

$$
\begin{aligned}
P_{v}^{\mu+1}(\operatorname{ch} \tau) & =\frac{(v-\mu) \operatorname{ch} \tau P_{v}^{\mu}(\operatorname{ch} \tau)-(v+\mu) P_{v-1}^{\mu}(\operatorname{ch} \tau)}{\operatorname{sh} \tau}, \\
(v-\mu+1) P_{v+1}^{\mu}(\operatorname{ch} \tau) & =(2 v+1) \operatorname{ch} \tau P_{v}^{\mu}(\operatorname{ch} \tau)-(v+\mu) P_{v-1}^{\mu}(\operatorname{ch} \tau) \\
\left(z^{2}-1\right) \frac{d P_{v}^{\mu}(z)}{d z} & =(v+\mu)(v-\mu+1)\left(z^{2}-1\right)^{1 / 2} P_{v}^{\mu-1}(z)-\mu z P_{v}^{\mu}(z) . \\
\left(z^{2}-1\right) \frac{d P_{v}^{\mu}(z)}{d z} & =v z P_{v}^{\mu}(z)-(v+\mu) P_{v-1}^{\mu}(z) .
\end{aligned}
$$

All of these formulas are used to calculate the values $P_{\nu}^{\mu}(\operatorname{ch} \tau)$ and $Q_{\nu}^{\mu}(\operatorname{ch} \tau)$ for all $\tau>0$ and $(\mu, v) \in \mathbb{C}^{2}$.

If $\mu$ and $\tau$ are fixed, the following proposition describes the behavior of associated Legendre functions of the first and second kind when $v=n-1 / 2, n \in \mathbb{Z}$, as $|n| \rightarrow+\infty$.

Proposition 8.1 Fix $\tau>0$ and $\mu \in \mathbb{C}$. Then if $v=n-1 / 2$ and $n \in \mathbb{Z}$, we have:

$$
\begin{aligned}
P_{\nu}^{\mu}(\operatorname{ch} \tau) & \sim \frac{e^{\tau / 2}}{\sqrt{2 \pi \operatorname{sh} \tau}} v^{\mu-1 / 2} e^{\tau \nu} & \text { as } v \rightarrow+\infty, \\
P_{\nu}^{\mu}(\operatorname{ch} \tau) & \sim \frac{e^{-\tau / 2}}{\sqrt{2 \pi \operatorname{sh} \tau}}(-v)^{\mu-1 / 2} e^{-\tau \nu} & \text { as } \quad v \rightarrow-\infty, \\
Q_{\nu}^{\mu}(\operatorname{ch} \tau) & \sim e^{i \pi \mu} e^{-\tau / 2} \sqrt{\frac{\pi}{2 \operatorname{sh} \tau}} v^{\mu-1 / 2} e^{-\tau \nu} & \text { as } \quad v \rightarrow+\infty, \\
Q_{\nu}^{\mu}(\operatorname{ch} \tau) & \sim e^{i \pi \mu} e^{\tau / 2} \sqrt{\frac{\pi}{2 \operatorname{sh} \tau}}(-v)^{\mu-1 / 2} e^{\tau \nu} & \text { as } \quad v \rightarrow-\infty .
\end{aligned}
$$

These equivalences are locally uniform with respect to the variable $\tau$, i.e. uniform on the whole interval $\left[\tau_{0}, \tau_{1}\right]$ with $0<\tau_{0}<\tau_{1}$.

Proof If $v=n-1 / 2$ and $n \in \mathbb{N}$, see [50, p. 48], we have

$$
\begin{gathered}
P_{v}^{\mu}(\operatorname{ch} \tau)=\frac{\Gamma(v+1)}{\Gamma(v-\mu+1)} \frac{1}{\sqrt{2 \pi(v+1) \operatorname{sh} \tau}}\left[e^{(v+1 / 2) \tau}+e^{-\pi i(\mu-1 / 2)-(v+1 / 2) \tau}\right] \\
\cdot\left[1+\mathrm{O}\left(\frac{1}{v}\right)\right]
\end{gathered}
$$

By the Stirling formula as $v \rightarrow+\infty$

$$
\begin{aligned}
\frac{\Gamma(\nu+1)}{\Gamma(v-\mu+1)} & \sim \frac{\sqrt{2 \pi} v^{\nu+1 / 2} e^{-v}}{\sqrt{2 \pi}(\nu-\mu)^{\nu-\mu+1 / 2} e^{-v+\mu}}=\left(\frac{v}{v-\mu}\right)^{v+1 / 2}(v-\mu)^{\mu} e^{-\mu} \\
& =(v-\mu)^{\mu} e^{-\mu} \exp \left(-\left(v+\frac{1}{2}\right) \ln \left(1-\frac{\mu}{v}\right)\right) \sim v^{\mu}
\end{aligned}
$$


consequently,

$$
P_{\nu}^{\mu}(\operatorname{ch} \tau) \sim v^{\mu} \frac{1}{\sqrt{2 \pi \nu \operatorname{sh} \tau}} e^{\tau / 2} e^{\tau \nu}=\frac{e^{\tau / 2}}{\sqrt{2 \pi \operatorname{sh} \tau}} v^{\mu-1 / 2} e^{\tau \nu},
$$

which gives us the first estimate.

The second estimate is obtained thanks to the relation $P_{v}^{\mu}=P_{-v-1}^{\mu}$. The third estimate follows from [50, Formula (8.3)]:

$$
Q_{\nu}^{\mu}(\operatorname{ch} \tau) \sim \sqrt{\frac{\pi}{2 \operatorname{sh} \tau}} v^{\mu-1 / 2} e^{i \pi \mu} e^{-\tau(v+1 / 2)},
$$

and the last estimate follows from the fact that for $v=n-1 / 2$ and $n \in \mathbb{Z}$, we have

$$
Q_{-v-1}^{\mu}=Q_{v}^{\mu}
$$

The local uniformity of these equivalences implies from explicit expressions of $P_{v}^{\mu}$ and $Q_{v}^{\mu}$ in terms of hypergeometric functions available in [21, pp. 124-138] and estimates of these hypergeometric functions which are locally uniform [50, pp. 178-182].

Acknowledgments Both authors thank Laurent Baratchart and Alexander Borichev for very useful discussions and remarks on the preliminary version of this paper.

\section{References}

1. Ablowitz, M.J., Fokas, A.S.: Complex Variables. Cambridge Texts in Applied Mathematics. Cambridge University Press, Cambridge (1997)

2. Abramowitz, M., Stegun, I.A. (eds.): Handbook of Mathematical Functions. Dover, New York (1992)

3. Alladio, F., Crisanti, F.: Analysis of MHD equilibria by toroidal multipolar expansions. Nuclear Fusion 26(9), 1143-1164 (1986)

4. Axler, S., Bourdon, P., Ramey, W.: Harmonic Function Theory. Graduate Texts in Mathematics, vol. 137, 2nd edn. Springer, New York (2001)

5. Baratchart, L., Borichev, A., Chaabi, S.: Pseudo-holomorphic functions at the critical exponent. J. Eur. Math. Soc. (2013, to appear). arXiv:1309.3079

6. Baratchart, L., Fischer, Y., Leblond, J.: Dirichlet/Neumann problems and Hardy classes for the planar conductivity equation. Complex Var. Elliptic Equ. 59(4), 504-538 (2014)

7. Baratchart, L., Leblond, J., Rigat, S., Russ, E.: Hardy spaces of the conjugate Beltrami equation. J. Funct. Anal. 259(2), 384-427 (2010)

8. Blum, J.: Numerical Simulation and Optimal Control in Plasma Physics. Wiley/Gauthier-Villars Series in Modern Applied Mathematics. John Wiley \& Sons, Chichester (1989)

9. Blum, J., Boulbe, C., Faugeras, B.: Reconstruction of the equilibrium of the plasma in a Tokamak and identification of the current density profile in real time. J. Comput. Phys. 231(3), 960-980 (2012)

10. Brelot, M.: Équation de Weinstein et potentiels de Marcel Riesz. In: Hirsch, E., Mokobodzki, G. (eds.) Séminaire de Theorie du Potentiel 3. Lecture Notes in Mathematics, vol. 681, pp. 18-38. Springer, Berlin (1978)

11. Brelot-Collin, B., Brelot, M.: Représentation intégrale des solutions positives de l'équation $L_{k}(u)=$ $\sum_{1}^{n} \partial^{2} u / \partial x_{i}^{2}+\left(k / x_{n}\right) \partial u / \partial x_{n}=0$ ( $k$ constante réelle) dans le demi-espace $E\left(x_{n}>0\right)$, de $\mathbb{R}^{n}$. Acad. Roy. Belg. Bull. Cl. Sci. (5) 58, 317-326 (1972)

12. Brelot-Collin, B., Brelot, M.: Allure à la frontière des solutions positives de l'équation de Weinstein $L_{k}(u)=\Delta u+\left(k / x_{n}\right) \partial u / \partial x_{n}=0$ dans le demi-espace $E\left(x_{n}>0\right)$ de $\mathbb{R}^{n}(n \geq 2)$. Acad. Roy. Belg. Bull. Cl. Sci. (5) 59, 1100-1117 (1973) 
13. Brelot-Collin, B., Brelot, M.: Étude à la frontière des solutions locales positives de l'équation (1) $L_{k}(u)=\Delta u+\left(k / x_{n}\right) \partial u / \partial x_{n}=0$ dans le demi-espace $E\left(x_{n}>0\right)$ de $\mathbb{R}^{n}(n \geq 2)$. Acad. Roy. Belg. Bull. Cl. Sci. (5) 62, 322-340 (1976)

14. Chalendar, I., Partington, J.R.: Phragmén-Lindelöf principles for generalized analytic functions on unbounded domains. Complex Anal. Oper. Theory. doi:10.1007/s11785-015-0453-z

15. Cohl, H.S., Tohline, J.E., Rau, A.R.P., Srivastava, H.M.: Developments in determining the gravitational potential using toroidal functions. Astron. Nachr. 321(5-6), 363-372 (2000)

16. Copson, E.T.: On sound waves of finite amplitude. Proc. Roy. Soc. London. Ser. A. 216(1127), 539-547 (1953)

17. Copson, E.T.: On Hadamard's elementary solution. Proc. Roy. Soc. Edinburgh. Sect. A 69(1), 19-27 (1970)

18. Copson, E.T.: Partial Differential Equations. Cambridge University Press, Cambridge (1975)

19. Dautray, R., Lions, J.-L.: Analyse Mathématique et Calcul Numérique pour les Sciences et les Techniques, vol. II. Collection Enseignement, Masson, Paris (1987)

20. Diaz, J.B., Weinstein, A.: On the fundamental solutions of a singular Beltrami operator. In: Studies in Mathematics and Mechanics Presented to Richard von Mises, pp. 97-102. Academic Press, New York (1954)

21. Erdélyi, A. (ed.): Higher Transcendental Functions, vol. I. McGraw-Hill, New York (1953)

22. Erdélyi, A.: Singularities of generalized axially symmetric potentials. Commun. Pure Appl. Math. 9(3), 403-414 (1956)

23. Erdélyi, A.: An application of fractional integrals. J. Anal. Math. 14, 113-126 (1965)

24. Erdélyi, A.: Axially symmetric potentials and fractional integration. J. Soc. Indust. Appl. Math. 13(1), 216-228 (1965)

25. Fischer, Y., Leblond, J.: Solutions to conjugate Beltrami equations and approximation in generalized Hardy spaces. Adv. Pure Appl. Math. 2(1), 47-63 (2011)

26. Fischer, Y., Leblond, J., Partington, J.R., Sincich, E.: Bounded extremal problems in Hardy spaces for the conjugate Beltrami equation in simply-connected domains. Appl. Comput. Harmon. Anal. 31(2), 264-285 (2011)

27. Fischer, Y., Marteau, B., Privat, Y.: Some inverse problems around the tokamak Tore Supra. Commun. Pure Appl. Anal. 11(6), 2327-2349 (2012)

28. Garabedian, P.R.: Partial Differential Equations. AMS Chelsea, Providence (1998)

29. Gilbert, R.P.: Some properties of generalized axially symmetric potentials. Amer. J. Math. 84(3), 475-484 (1962)

30. Gilbert, R.P.: On generalized axially symmetric potentials. J. Reine Angew. Math. 212, 158-168 (1963)

31. Gilbert, R.P.: Poisson's equation and generalized axially symmetric potential theory. Ann. Mat. Pura Appl. 61, 337-348 (1963)

32. Gilbert, R.P.: Bergman's integral operator method in generalized axially symmetric potential theory. J. Math. Phys. 5, 983-997 (1964)

33. Hall, N.S., Quinn, D.W., Weinacht, R.J.: Poisson integral formulas in generalized bi-axially symmetric potential theory. SIAM J. Math. Anal. 5(1), 111-118 (1974)

34. Henrici, P.: On the domain of regularity of generalized axially symmetric potentials. Proc. Amer. Math. Soc. 8(1), 29-31 (1957)

35. Hörmander, L.: The Analysis of Linear Partial Differential Operators, Vol. I. Grundlehren der Mathematischen Wissenschaften, vol. 256. Springer, Berlin (1990)

36. Huber, A.: A theorem of Phragmén-Lindelöf type. Proc. Amer. Math. Soc. 4(6), 852-857 (1953)

37. Huber, A.: On the uniqueness of generalized axially symmetric potentials. Ann. Math. 60(2), 351-358 (1954)

38. Huber, A.: Some results on generalized axially symmetric potentials. In: Proceedings of the Conference on Differential Equations, pp. 147-155. University of Maryland Book Store, College Park (1956)

39. Lebedev, N.N.: Special Functions and their Applications. Prentice-Hall, Englewood Cliffs (1965)

40. Lenells, J., Fokas, A.S.: Boundary-value problems for the stationary axisymmetric Einstein equations: a rotating disc. Nonlinearity $\mathbf{2 4}(1), 177-206$ (2011)

41. Liu, H.: The Cauchy problem for an axially symmetric equation and the Schwarz potential conjecture for the torus. J. Math. Anal. Appl. 250(2), 387-405 (2000)

42. Love, J.D.: The dielectric ring in a uniform, axial, electrostatic field. J. Math. Phys. 13, 1297-1304 (1972) 
43. Nikolski, N.K.: Operators, Functions, and Systems, Vol. II. Mathematical Surveys and Monographs, vol. 93. American Mathematical Society, Providence (2002)

44. Savina, T.V.: On splitting up singularities of fundamental solutions to elliptic equations in $\mathbb{C}^{2}$. Cent. Eur. J. Math. 5(4), 733-740 (2007)

45. Segura, J., Gil, A.: Evaluation of toroidal harmonics. Comput. Phys. Commun. 124(1), 104-122 (2000)

46. Shafranov, V.D.: On magnetohydrodynamical equilibrium configurations. Soviet Phys. JETP 6(3), 545-554 (1958)

47. Shushkevich, G.Ch.: Electrostatic problem for a torus and a disk. Technical Phys. 42(4), 436-438 (1997)

48. van Milligen, B.Ph., Lopez Fraguas, A.: Expansion of vacuum magnetic fields in toiroidal harmonics. Comput. Phys. Commun. 81(1-2), 74-90 (1994)

49. Vekua, I.N.: New Methods for Solving Elliptic Equations. North-Holland Series in Applied Mathematics and Mechanics, vol. 1. North-Holland, Amsterdam (1967)

50. Virchenko, N., Fedotova, I.: Generalized Associated Legendre Functions and their Applications. World Scientific, River Edge (2001)

51. Weinacht, R.J.: Fundamental solutions for a class of singular equations. Contributions to Differential Equations 3, 43-55 (1964)

52. Weinacht, R.J.: A mean value theorem in generalized axially symmetric potential theory. Atti Accad. Naz. Lincei Rend. Cl. Sci. Fis. Mat. Natur. 8(38), 610-613 (1965)

53. Weinacht, R.J.: Fundamental solutions for a class of equations with several singular coefficients. J. Austral. Math. Soc. 8(3), 575-583 (1968)

54. Weinstein, A.: Discontinuous integrals and generalized potential theory. Trans. Amer. Math. Soc. 63(2), 342-354 (1948)

55. Weinstein, A.: On generalized potential theory and on the torsion of shafts. In: Studies and Essays Presented to R. Courant on his 60th Birthday, pp. 451-460. Interscience, New York (1948)

56. Weinstein, A.: On the torsion of shafts of revolution. In: Proceedings of the Seventh International Congress on Applied Mechanics, vol. 1, pp. 108-119 (1948)

57. Weinstein, A.: Transonic flow and generalized axially symmetric potential theory. In: Symposium on Theoretical Compressible Flow, pp. 73-82. Naval Ordnance Laboratory, White Oak (1950)

58. Weinstein, A.: On Tricomi's equation and generalized axially symmetric potential theory. Acad. Roy. Belgique. Bull. Cl. Sci. 5(37), 348-358 (1951)

59. Weinstein, A.: Generalized axially symmetric potential theory. Bull. Amer. Math. Soc. 59(1), 20-38 (1953)

60. Weinstein, A.: The singular solutions and the Cauchy problem for generalized Tricomi equations. Commun. Pure Appl. Math. 7(1), 105-116 (1954)

61. Weinstein, A.: On a class of partial differential equations of even order. Ann. Mat. Pura Appl. 39, 245-254 (1955)

62. Weinstein, A.: The generalized radiation problem and the Euler-Poisson-Darboux equation. Summa Brasil. Math. 3, 125-147 (1955)

63. Weinstein, A.: The method of axial symmetry in partial differential equations. In: Convegno Internazionale sulle Equationi Lineari alle Derivate Parziali, pp. 86-96. Edizioni Cremonese, Roma (1955)

64. Weinstein, A.: Elliptic and hyperbolic axially symmetric problems. In: Proceedings of the International Congress of Mathematicians, vol. 3, pp. 264-269. North-Holland, Amsterdam (1956)

65. Weinstein, A.: Sur une classe d'équations aux dérivées partielles singulières. In: Colloques Internationaux du Centre National de la Recherche Scientifique, vol. 71, pp. 179-186. Centre National de la recherche Scientifique, Paris (1956)

66. Weinstein, A.: On a singular differential operator. Ann. Mat. Pura Appl. 49, 359-365 (1960)

67. Weinstein, A.: Singular partial differential equations and their applications. In: Fluid Dynamics and Applied Mathematics, pp. 29-49. Gordon and Breach, New York (1962)

68. Weinstein, A.: Some applications of generalized axially symmetric potential theory to continuum mechanics. Applications of the Theory of Functions in Continuum Mechanics, vol. 2, pp. 440-453. Nauka, Tbilisi (1965) 